\title{
Immunometabolism in biofilm infection: lessons from cancer
}

\author{
Rasoul Mirzaei ${ }^{1,2^{*}}$ (D) Niloofar Sabokroo ${ }^{3}$, Yaghoub Ahmadyousefi ${ }^{4,5}$, Hamid Motamedi ${ }^{6}$ and Sajad Karampoor ${ }^{7,8^{*}}$
}

\begin{abstract}
Background: Biofilm is a community of bacteria embedded in an extracellular matrix, which can colonize different human cells and tissues and subvert the host immune reactions by preventing immune detection and polarizing the immune reactions towards an anti-inflammatory state, promoting the persistence of biofilm-embedded bacteria in the host.

Main body of the manuscript: It is now well established that the function of immune cells is ultimately mediated by cellular metabolism. The immune cells are stimulated to regulate their immune functions upon sensing danger signals. Recent studies have determined that immune cells often display distinct metabolic alterations that impair their immune responses when triggered. Such metabolic reprogramming and its physiological implications are well established in cancer situations. In bacterial infections, immuno-metabolic evaluations have primarily focused on macrophages and neutrophils in the planktonic growth mode.

Conclusion: Based on differences in inflammatory reactions of macrophages and neutrophils in planktonic- versus biofilm-associated bacterial infections, studies must also consider the metabolic functions of immune cells against biofilm infections. The profound characterization of the metabolic and immune cell reactions could offer exciting novel targets for antibiofilm therapy.
\end{abstract}

Keywords: Biofilm, Biofilm infection, Cancer, Metabolism, Immune polarization, Immunometabolism

\section{Background}

Biofilm has been characterized as an accumulation of microorganisms embedded in extracellular polymeric substances (EPS) and attached to biotic or abiotic surfaces (Flemming et al. 2016, Mirzaei et al. 2020a; Mirzaei et al. 2020b). Biofilm has emergent attributes and novel properties that are not predictable from the survey of planktonic bacteria. Biofilm is one of the most broadly successful and distributed forms of life (Stoodley et al. 2002; Mahdiun et al. 2017). Bacterial biofilm is

*Correspondence: rasul.micro92@gmail.com; sajadkarampour1987@gmail. com

${ }^{1}$ Department of Microbiology, School of Medicine, Hamadan University of Medical Sciences, Hamadan, Iran

${ }^{8}$ Department of Virology, School of Medicine, Iran University of Medical Sciences, Tehran, Iran

Full list of author information is available at the end of the article highly resistant to antibacterial drugs and host immune responses (Stoodley et al. 2002). It has been demonstrated that $80 \%$ of all bacterial infections in humans are associated with biofilm (Costerton et al. 1999; Mirzaei et al. 2020c).

The interplay among prokaryotes and eukaryotes is typical (Hakansson et al. 2018, 2022). This relationship affects the participants' metabolism on different paths, culminating in neutral, advantageous, and/or detrimental results for the members (Escoll and Buchrieser 2019). However, these fruitful interactions are less lasting and can potentially disrupt different agents, such as bacterial pathogens (Lupp et al. 2007). Metabolic changes in eukaryotic cells following different serious biofilm-associated infections are sometimes contradictory Mirzaei et al. (2020d). Frequent metabolic changes can occur in 
pathogens and host cells across biofilm-associated diseases (Molinero et al. 2019b).

On the other hand, the significant impact of tumor cells' metabolic changes and disease progression is well known, and metabolic reprogramming has been deemed one of cancer's hallmarks (Guo et al. 2019). The shift in main metabolic processes inside immune cells is now perceived to be due to nutrients or oxygen status and immune stimuli. It is evident that distinctive metabolic pathways, other than energy generation and biosynthetic pathways, control the shape and functions of immune cells (Guo et al. 2019; Mirzaei et al. 2021a; 2019). Physiological evaluations of bacterial infections showed that there is a close relationship between the host metabolic system and the host immune system (Belkaid and Hand 2014). This interaction begins soon after detecting pathogens and continues to evolve during their infection (Belkaid and Hand 2014). The current interest in the interface between host metabolism and host immunity has given rise to the field of 'Immunometabolism' (Guo et al. 2019, Mirzaei et al. 2021b). The host immune reaction generated in the biofilm infection is mostly ineffective, which results in chronic infections (Campoccia et al. 2019, 2020). This has been shown to occur through various pathways, which comprise direct encounters of neutrophils, macrophages, and myeloid-derived suppressor cells (MDSCs) (Rada 2015, 2017; Heim et al. 2014). Determining whether and how biofilm prevents host immune-mediated killing can help advance therapeutic approaches to enhance proinflammatory responses and facilitate the clearance of biofilm infections. Currently, it has known that alterations in the metabolism of immune cells can shape their function and phenotype (O'Neill and Pearce 2016; Loftus and Finlay 2016). For example, antiinflammatory macrophages use oxidative phosphorylation (OxPhos), whereas pro-inflammatory macrophages favor aerobic glycolysis (Benoit and Koo 2016). This review will summarize the immune cells' metabolic reactions during biofilm infections compared with cancer.

\section{Biofilm and cancer: similarities and links}

It has been documented that bacteria are encountered with various physiological states like anaerobic and dormancy based on the availability of micronutrients, $\mathrm{pH}$, oxygenation, and bacterial metabolites generated inside biofilm (Maali et al. 2020). Following biofilm maturation, some aggregated bacteria could detach that, allowing colonization on other sites, resulting in the metastasis of the biofilm infection (Ivanenko 2021). Additionally, the extracellular milieu is a significant property that controls the function of bacterial and tumor cells alike. In this regard, for tumors, the extracellular matrix is usually changed and disorganized based on acidic $\mathrm{pH}$ and local hypoxia areas that impact the progression of the tumor (Petrova et al. 2018). Also, this extracellular matrix can be fibrotic and denser as tumors enlarge, resulting in limiting diffusion (Petrova et al. 2018). Taken together, the dysfunctional matrix causes diffusive barriers leading to micro-environmental heterogeneity that stops drug distribution (Ivanenko 2021). On the other hand, the microbial EPS matrix of biofilm also generates heterogeneities, localized $\mathrm{pH}$, and oxygen gradients microenvironments, changing the bacterial survival and virulence (Koo et al. 2013).

The treatment of biofilm infections and solid tumors faces a similar problem; for example, drugs usually fail to reach and kill tumors and biofilm-embedded bacteria due to local micro-environment heterogeneities (Benoit and Koo 2016). Besides, traditional systemic and topical therapeutic approaches could be toxic cause significant damage to healthy tissues (Benoit and Koo 2016). For example, in cancer, low red blood cell, platelet, neutrophil, and immune cell counts are common side effects for chemotherapy, while ototoxicity, nephrotoxicity, and neuromuscular blockade are common in antimicrobials against biofilm (Ivanenko 2021). Also, treatments associated with biofilm, including administration of high-dose antibiotics, can cause substantial collateral damage such as microbiota dysbiosis, resulting in the proliferation of bacterial pathogens (Benoit and Koo 2016). Taken together, cancer and biofilm show several unique similarities that could be considered to design new therapeutic approaches (Ivanenko 2021; Benoit 2016). Most importantly, altered immune reactions are usually noted for biofilm and tumor microenvironments (Ivanenko 2021; Benoit 2016). Altogether, there are crucial challenges for traditional antibiofilm and anticancer drugs to overcome these situations where pathogens and cancer cells survive and cause the onset of disorders (Ivanenko 2021; Benoit 2016).

It has been found that the ability of biofilm-embedded bacteria to evade antibiotics is mainly because of persisters, resistance, and tolerance (Lebeaux et al. 2014). In this regard, persister and tolerant bacteria are noted for difficulties in biofilm-associated chronic infections (Yan and Bassler 2019). Also, the similar parallel between biofilm infections and cancer shows that anti-persister approaches could help administer cancer treatment (Ivanenko 2021). For example, the inhibition of lipid hydroperoxidas Glutathione Peroxidase (GPX4) prevents the persistence of cells and results in the prevention of drug resistance in cancers (Ivanenko 2021). Better characterization of the behavior of various cell states in microbial biofilm could cause a reduction in 
the ineffectiveness of current therapeutic choices for both biofilm infection and cancer. The overview of similarities in cancer and biofilm is depicted in Table 1.

Currently, some studies have found compositional changes in the tissue-mediated microorganisms in colorectal cancer individuals, for example, enterotoxigenic Bacteroides fragilis (ETBF), Enterobacteriaceae carrying the genotoxic polyketide synthase (pks), as well as Fusobacterium nucleatum (Ivanenko 2021). Nevertheless, the precise interplays between biofilm composition and cancer are being raised in this regard. Enterobacteriaceae and B. fragilis have been found inside mucosal biofilm from biopsies collected from individuals with inflammatory bowel disease (IBD), showing intestinal inflammation also impacts host susceptibility (Tomkovich et al. 2018). Fecal samples from individuals with carcinoma are enriched in Parabacteroides, Bacteroides, as well as Escherichia (Ivanenko 2021). Importantly, it has been found further link between biofilm-associated bacteria and colorectal cancer (Fig. 1) (Rizzato et al. 2019). In this regard, a study found polymicrobial biofilm production in colorectal mucosa of individuals with colorectal cancer (Rizzato et al. 2019). This phenomenon was striking for right-sided tumors compared to left-sided tumors (Dejea et al. 2014; Drewes et al. 2017). Interestingly, biofilm was present on tumors and at normal surgical margins. Besides, biofilm production was associated with reduced colonic epithelial cell E-cadherin, increased Interleukin-6 (IL-6) and Signal transducer and activator of transcription 3 (STAT3) stimulation, and enhanced crypt cell growth in normal colon mucosa (Dejea et al. 2014). Further analysis showed that these tumor-mediated biofilms were enriched with $B$. fragilis, Peptostreptococcus stomatits, and F. nucleatum (Rada 2017). Also, biofilm was found in colorectal mucosa of genetically predisposed patients, and it was rather patchy and primarily made of $B$. fragilis and polyketide-peptide genotoxin forming pks island positive Escherichia coli (Rizzato et al. 2019; Dejea et al. 2014). Additionally, it was also noted previously that $B$. fragilis is a crucial part of IBD-associated biofilm (Swidsinski et al. 2005).

Another bit of corroborative knowledge emerges from the increasing perception of the correlation between the oral microbiome, specifically $F$. nucleatum, and colorectal cancer, which was predominantly noted for proximal colon tumors (Sun and Kato 2016; Hussan et al. 2017). F. nucleatum is a routine residential part of dental plaques (biofilms) and prominent periodontal pathogen (Larsen and Fiehn 2017). F. nucleatum is deemed a co-aggregation expert, with an ability to co-aggregate with a wide range of bacteria, approximately all bacterial species implicated in oral plaque development, a crucial feature in biofilm formation (Kolenbrander et al. 1989; Allen-Vercoe et al.
2011). Besides, F. nucleatum can attach to and transport contrarily non-invasive bacterial species within host cells, working as a shuttle in this regard (Edwards et al. 2006). F. nucleatum constructs outer membrane vesicles (OMV) to promote co-aggregation, and separate OMVs only have been showed to exert an equivalent capacity to co-aggregate other bacteria examined to the complete bacterial culture (Kinder and Holt 1993). OMV generation seems to depend on external motives, and producing alterations in biofilm development are strain-specific (Martins et al. 2016). In this setting, the presumed correlation with colorectal cancer may perform the microbes that $F$. nucleatum associates in its biofilms rather than directly impacting its virulence. Flemer et al. (2018) recently described that various microbes regularly found in oral biofilms were enhanced in colonic mucosa from colorectal cancer cases. Ultimately, $F$. nucleatum has lately been discovered to be one of the gut microbes connected to pancreatic cancer, although limited known regarding biofilm production in the pancreatic ducts (Castillo et al. 2019). Further, investigations reveal that the commensal (such as Parvimonas, Peptostreptococcus, Prevotella) and the pathogenic (such as $F$. nucleatum, $P$. gingivalis) periodontal bacteria, which are able of creating biofilms, are identified in the intestinal biofilms (Drewes et al. 2017; Donelli et al. 2012). Hence, an intriguing hypothesis was intended to demonstrate the potential engagement of oral microbiota in colorectal cancer development. According to intestinal dysbiosis, the oral periodontopathic bacteria may have translocated within the colorectum. This introduces a fresh outlook on colorectal cancer pathogenesis which is operated by the orally-derived colonic biofilm (Koliarakis et al. 2019).

Intriguingly, a current murine investigation by Tomkovich et al. (2019), designed to portray the causality of microbial biofilms in colorectal cancer, strongly displayed that the polymicrobial biofilms are carcinogenic in a preclinical in vivo experimentation with the application of three murine genetic models of colorectal cancer carcinogenesis. An impressive conclusion of this research was that biofilm communities from the colon biopsies of healthy people were as robust as biofilm communities from colorectal cancer hosts in inducing the increase of tumors (Tomkovich et al. 2019). Moreover, the newest finding further determined that the same levels of inflammation were recognized in both mice treated with biofilm-positive and biofilm-negative control homogenates. Nevertheless, a lower grade of immunosuppressive myeloid cell recruitment and Interleukin-17 (IL-17) generation was triggered by biofilm-negative control homogenates when contrasted to biofilm-positive homogenates in the mice (Tomkovich et al. 2019). 


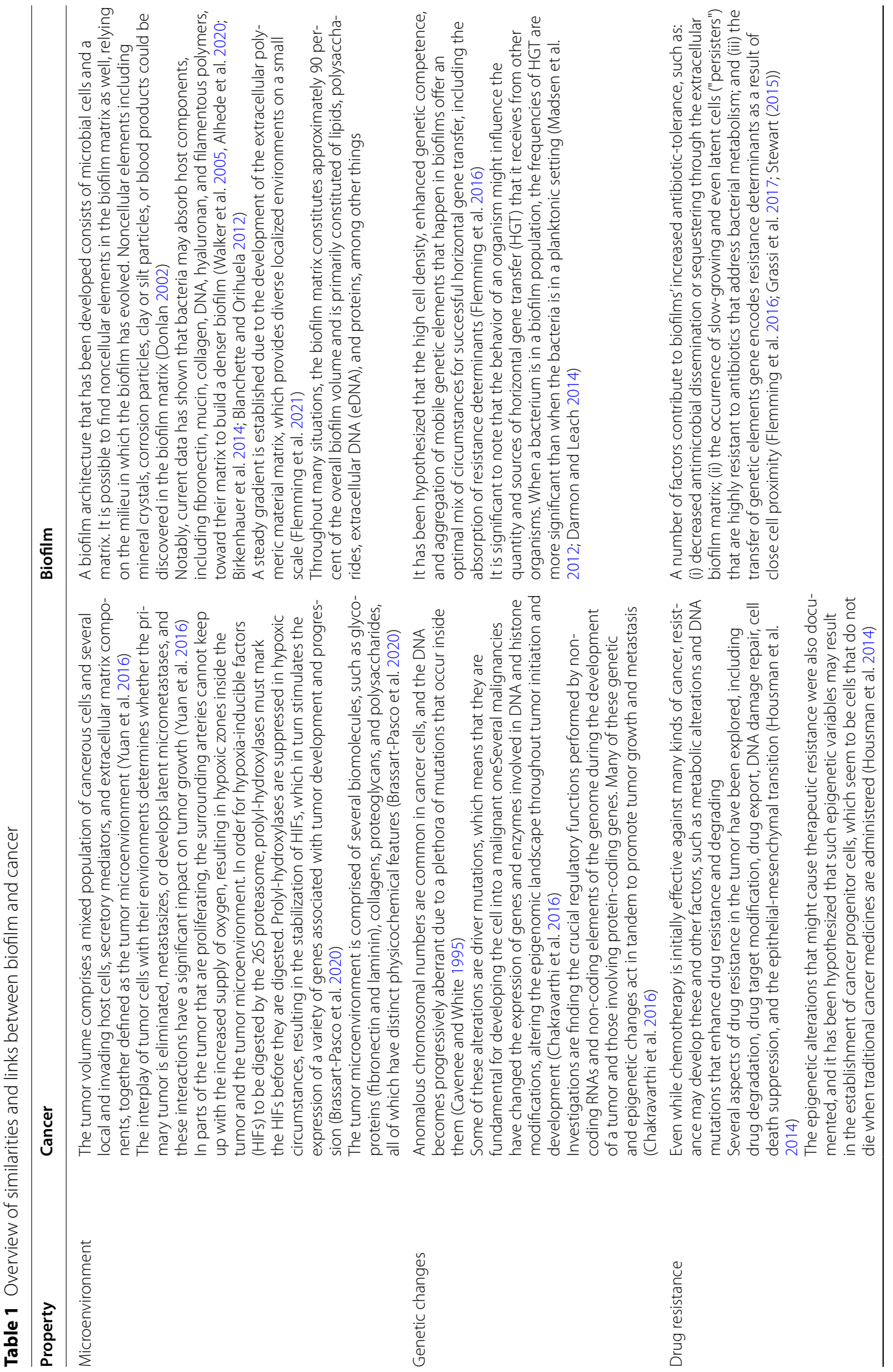




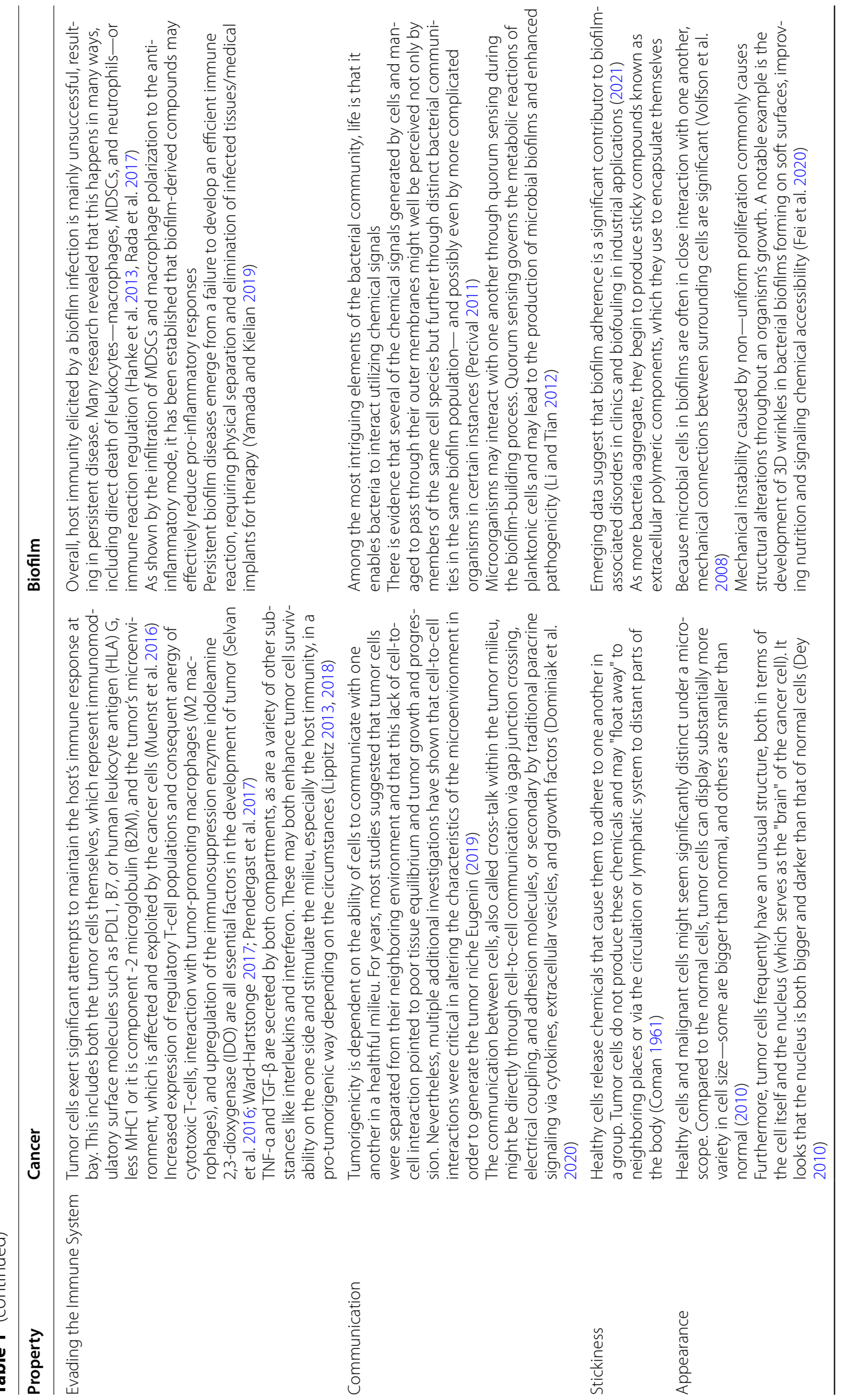




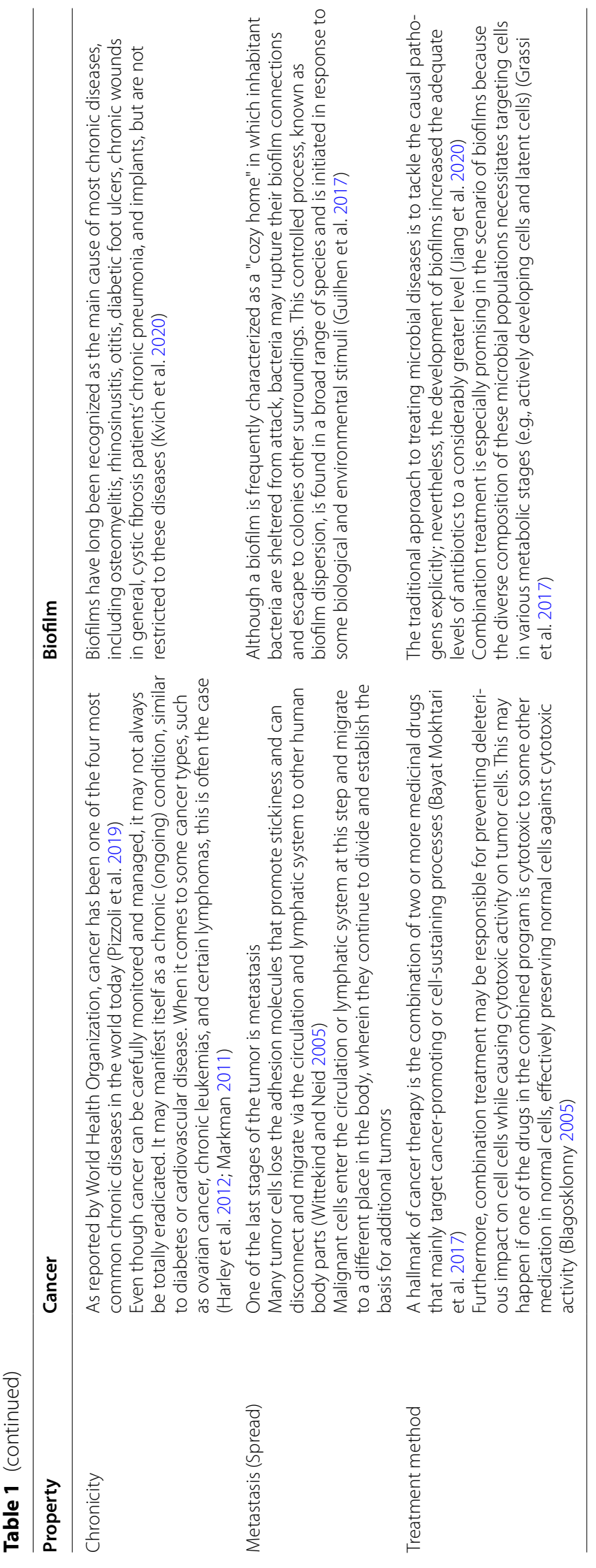




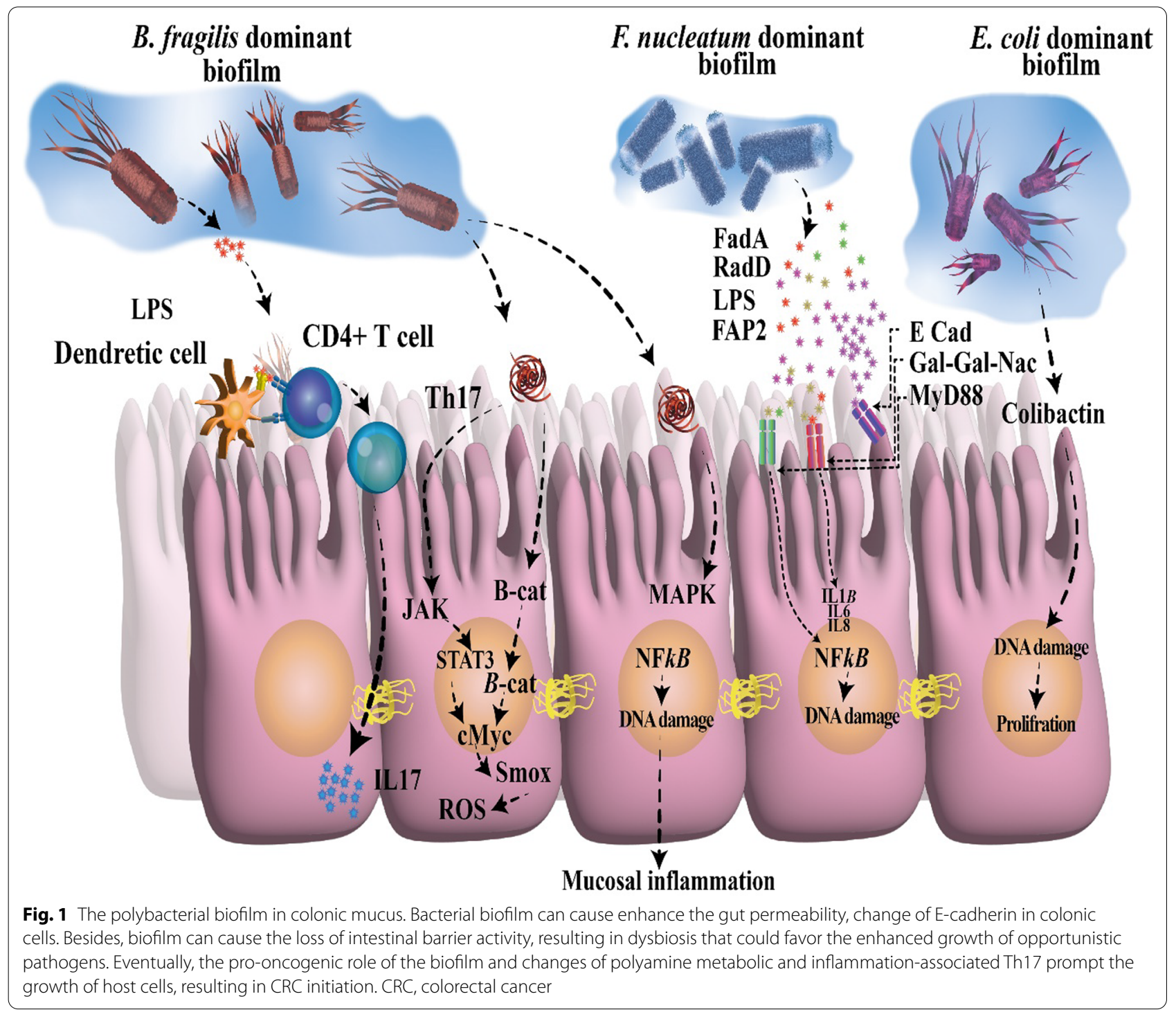

\section{Helicobacter pylori biofilm and cancer}

The gastric lumen is one of the common hostile circumstances in the human body, eliminating various bacteria in a few minutes. Helicobacter pylori (H. pylori) persist in this environment with its urease activity compensating gastric acid, whereas biofilm development may be further necessary for its persistent colonization (Abadi 2017). Surprisingly, concisely has been acknowledged concerning in vivo biofilm production on the human gastric mucosa to date. Three pioneer investigations operated a high-powered electron microscope and displayed thick clusters of $H$. pylori, originally in coccoid forms, which are identified as viable but non-cultivable (Carron et al. 2006; Coticchia et al. 2006; Cellini et al. 2008; Cellini 2014; Percival and Suleman 2014). It is fascinating to perceive that $H$. pylori isolated from gastric cancer cases are usually non-cultivable, also though the bacteria are detectable by distinct methods (i.e., polymerase chain reaction (PCR) or histology), and that coccoid forms have been certainly more commonly observed in gastric mucosa of gastric cancer cases than in that of peptic ulcer subjects (Chan et al. 1994). Indeed, H. pylori are the primary risk factor for the growth of gastric adenocarcinoma, which happens in almost 1 to $2 \%$ of infected people (Parkin 2006; McColl 2010).

Following its identification in the 1980s, investigations of $H$. pylori have concentrated on the planktonic, free-floating, nonattached form of growth; nevertheless, the current sign implies that $H$. pylori can also grow in a surface-attached biofilm form (Hathroubi et al. 2018). Investigations are starting to clarify $H$. pylori biofilm growth both in vitro and in vivo (Carron et al. 2006; Hathroubi et al. 2018; Servetas et al. 2016). While 
investigating $H$. pylori biofilms is almost new, the study in this unique field has gradually gained momentum over the past few years. Given the chronicity correlated with $H$. pylori infection, it is possibly not unexpected that this bacterium is recognized in biofilm (Hathroubi et al. 2018). Given that inflammation performs a crucial function in gastric carcinogenesis and that $H$. pylori have beneficial impacts on extra-gastric tissues, it may be therapeutically efficient to fine-tune the attendance of $H$. pylori in its active position in tissues where it is useful and target its inflammatory function in tissue where it is dangerous (Mentis et al. 2019, 2019). This approach could align with investigations revealing the anti-inflammatory performance of probiotics and other digestive factors such as Mastiha gum against $H$. pylori (Paraschos et al. 2007). Honey and yogurt eating have additionally been noted to be correlated with declines in H. pylori and antiCagA Immunoglobulin G (IgG) seroprevalence, probably because of honey's antimicrobial activities and yogurt's probiotic and prebiotic actions (Yordanov and Boyanova 2017). It would be attractive to reveal if these results can be connected to biofilm activity, supposing that the quorum-sensing ability of biofilms can manage microbial community density.

The several procedures, including genomics, transcriptomics, and proteomics, are high-throughput that have been performed to recognize factors correlated with $H$. pylori biofilms (Hathroubi et al. 2018). One genomic investigation compared the sequences of wild-type strain 09 and $31 \mathrm{H}$. pylori clinical isolates that each had a spectrum of biofilm-forming capabilities as a method to distinguish genes enhanced in those strains that were high biofilm generators (Wong et al. 2016). The strains based on crystal violet staining intensity were categorized as low, moderate, or high biofilm generators (Wong et al. 2016). By analyzing the genetic diversity among strains, those contributors could identify several genes that seem to be correlated with biofilm production. Indeed, three hypothetical genes (K74_10375, K747_09130, and K747_06625) were remarkably associated with biofilm production (Wong et al. 2016). K747_06625 is prognosticated to hold a homing endonuclease and a ParB-like domain, the latter of which is correlated with biofilm development in some bacteria (Mashimo et al. 2013, 2014).

Additionally, four operative genes, coding for a flagellar protein (jhp_1117), an alpha-(1, 3)-fucosyltransferase, an outer membrane protein (OMP) (encoded by homD), and a cytotoxin-associated gene (Cag) pathogenicity island (PAI), were similarly correlated with biofilm development in H. pylori (Wong et al. 2016). The performance of the Cag PAI proteins in H. pylori biofilms was extra studied by the production of deletion mutations in CagA and the complete Cag PAI (Wong et al. 2016). Both mutations resulted in a notable reduction in biofilm biomass contrasted with that of the wild type (Wong et al. 2016). Additional work similarly advocated the significance of the Cag PAI proteins. Through employing a proteomic approach, Shao et al. (2013) recognized that two Cag proteins, Cag26/CagA and Cag24/CagD, were highly expressed in $H$. pylori biofilms provoked by serum deprivation related to planktonic $H$. pylori cultured below the same circumstances (Shao et al. 2013). Similarly, an investigation also revealed that the production of isogenic strains lacking CagE changed biofilm development by $H$. pylori, additional supporting the performance of the Cag PAI in biofilm production (Cole et al. 2004). These outcomes are especially relevant to pathogenesis because infections with Cag PAI-containing strains are usually correlated with an enhanced risk for gastric cancer (Nilsson et al. 2003).

\section{Host cellular metabolism during cancer and biofilm infection}

Mammalian cells depend on basic metabolic processes for energy generation, macromolecule biosynthetic pathways precursors, and redox control power reduction (Vander Heiden 2009). Glucose, fatty acids, and amino acids (in particular glutamine) are the primary nutrients supporting these processes (Cantor and Sabatini 2012). Glucose is glycolytic metabolism's favored carbon energy source to provide acetyl-CoA for oxidation by the mitochondrial tricarboxylic acid (TCA) cycle (Martínez-Reyes and Chandel 2020). The pentose phosphate pathway (PPP) branching off glycolysis offers an alternate path for glucose degradation by producing nicotinamide adenine dinucleotide phosphate (NADPH) and ribose-5-phosphate for nucleic acid biosynthesis (Guo et al. 2019). As blood glucose levels decrease, the fatty acids from deposited fat are separated into acetyl-CoA through fatty acid $\beta$ oxidation (FAO) in the mitochondrion (Schönfeld and Wojtczak 2016). This method is consistent with electron transfer to the electrons' transport chain to produce Adenosine Triphosphate (ATP) (Guo et al. 2019). The generated acetyl-CoA is applied to the TCA cycle or, more than the liver, recycled to form ketone bodies for extrahepatic tissues (Guo et al. 2019). Currently, it has been shown that FAO-formed acetyl-CoA enters the TCA cycle to form citrate, which can be conveyed to the cytoplasm to cause NADPH-generating isocitrate oxidation by isocitrate dehydrogenase (Carracedo et al. 2013, 2015; Williams and O'Neill 2018).

With the aid of novel cellular and molecular biological methods, cancer cell metabolism experiments have increased our knowledge of the pathways and functional effects of cancer-associated metabolic changes at different phases of carcinogenesis (Pavlova and Thompson 
2016). Of note, it has become clear that tumorigenesismediated metabolic alterations cover all phases of cellmetabolite interplay, (1) determining the metabolic flux by conferring an enhanced capacity to obtain the required micronutrients; (2) changing the way micronutrients are selectively transferred to metabolic processes that lead to cellular tumorigenesis characteristics (3) and exercising long-term impact on cell destiny, including modifications in the divergence of tumor cells themselves and the parts of the microenvironment of cancer (Pavlova and Thompson 2016). A cell for providing the biogenesis conditions correlated with growth must enhance nutrition intake from the surroundings (Pavlova and Thompson 2016). The two primary nutrients that enable longevity and biosynthesis in cells are glutamine and glucose (Pavlova and Thompson 2016). Via the catabolism of glucose and glutamine, the cell retains reservoirs of diverse carbon intermediates that are employed as fundamental elements for constructing various macromolecules (Pavlova and Thompson 2016). Besides, regulated oxidation of glucose and glutamine carbon skeletons enables the cell to catch the reducing power each in the form of NADH and Flavin adenine dinucleotide (FAD) $\mathrm{H} 2$, which relate to the transition of electrons to the transport chain of electrons for the making of ATP energy or in the form of a relevant NADPH cofactor, which supplies reducing power for a broad range of biosynthetic re-engines (Pavlova and Thompson 2016).

The German physiologist Otto Warburg initially identified a dramatically elevated glucose absorption by cancers relative to non-proliferating normal tissues (Warburg 1924; Warburg et al. 1927). This finding has been verified in several tumor conditions and is associated with a bad tumor prognosis (Som et al. 1980). Positron emission tomography-based visualization of the absorption of a radioactive fluorine-labeled glucose relative, 18 F-fluorodeoxyglucose, has been applied to detect and stage tumors, as well as for testing exposure to care (Almuhaideb et al. 2011). Glutamine, the second main growth-supporting substrate, involves carbon and decreased nitrogen for de novo production of a variety of various nitrogen-containing substances (Pavlova and Thompson 2016). Glutamine provides the nitrogen demanded by the biogenesis of pyrimidine and purine nucleotides, glucosamine-6-phosphate, as well as nonessential amino acids (Pavlova and Thompson 2016). Also, glutamine has been known to play an activity in absorbing essential amino acids (Pavlova and Thompson 2016). While non-essential amino acids could be generated by mammalian de novo cells, essential amino acids must be obtained from external sources (Pavlova and Thompson 2016). It is essential to notice that the import of critical amino acid leucine via the plasma membrane-located neutral amino acid antidote L-Type Amino Acid Transporter (LAT1) was found to be combined with the concurrent efflux of glutamine (Nicklin et al. 2009). In this way, intracellular glutamine can promote importing a wide range of LAT1 substrates, such as valine, leucine, isoleucine, tyrosine, methionine, phenylalanine, and tryptophan (Yanagida et al. 2001).

Host metabolic activities are studied in various disciplines, including aging, immunity, and cancer. These include the catabolic biochemical processes for energy production (ATP) and anabolic reactions to produce biomolecules (Vander Heiden and DeBerardinis 2017; Finkel 2015; Escoll and Buchrieser 2018). There is a two-way relationship between the metabolic status and activity of individual host cells (Escoll and Buchrieser 2018). Hence, various metabolic pathways exist in proliferating, and non-proliferating cells and non-differentiated and differentiated cells, and these pathways are executed at a particular time. They are crucial for the functional status of cells (Escoll and Buchrieser 2018). Interactions between prokaryotes and eukaryotic cells are common and can affect the nutrients' metabolism in the environment, leading to beneficial, neutral, and harmful effects on the host cell. These interactions also occur between the host and its diverse microbiome (Mithieux 2018). These communications are short and could be altered by pathogens (Lupp et al. 2007). The metabolic communications that occur in eukaryotic cells during biofilm infection are competitive, in which the host cells try to limit nutrient access to the biofilm bacteria.

In contrast, the bacteria defend by taking advantage of the host micro-nutrients and metabolites (Molinero et al. 2019a). Metabolic changes in host cell following biofilm infections are of growing importance for understanding the pathogenesis of bacterial biofilm infections. A growing body of studies has been performed, mainly focused on the inflammation and immune reactions, to elucidate host responses in biofilm infections. For example, the formation of endosomal vesicles, trafficking, autophagy, host cell survival, and apoptosis have been studied, while the effects of biofilm infections on host metabolism are less studied (Huang and Brumell 2014; Lazar et al. 2018; Molinero et al. 2019a; Zhang et al. 2017). Most of the work on host reactions is mainly associated with signaling pathways and enzymes or transcription factors that influence the metabolism of nitrogen, carbon, and energy metabolism (Eisenreich et al. 2013, 2019).

Bacteria are increasingly being found to be active in stimulating and promoting cancer, especially colon cancer. Dejea et al. (Eisenreich et al. 2013, 2019) have characterized a biofilm description and its metabolic involvement in colon cancer in pioneering work. They found that biofilm can alter the mode of epithelial cells 
concerning oncogenic progression including decreased tumor suppressors, increased levels of inflammatory and angiogenic cytokines decrease in E-cadherin, and activation of IL-6, and STAT3 (Dejea et al. 2014; Dejea and Sears 2016). The colon biofilm as a pathogenic form has previously been associated with Crohn disease, IBD, and ulcerative colitis. Dejea et al. (Dejea et al. 2014; Dejea and Sears 2016) discovered that biofilms from colon cancer alter epithelial cell proliferation and metabolism in this context. It has been shown that in colon cancer, the rate of $\mathrm{N}$ (1), $\mathrm{N}$ (12)-diacetylspermine was increased in response to biofilm (Dejea et al. 2014; Dejea and Sears 2016). Higher levels of different polyamines have been diagnosed in colon cancer where the molecules of $\mathrm{N}$ (1), N (12)-diacetylspermine were found in the urine of patients with colon cancer, suggesting direct linkage of polyamine metabolites and biofilm formation (Dejea et al. 2014; Dejea and Sears 2016). The idea that biofilm can influence different metabolite molecules that affect the carcinogenesis process is a new area that needs further research (Hiramatsu et al. 2005). The recognition that biofilm communities can control the risk of oncogenesis is a starting point for new lines of research that can provide great insight into the role of the microbiome in colon cancer.

\section{Immune polarization}

In vitro investigations of macrophage activation resulted in the discovery of M1 (classical) and M2 (alternative) modes for macrophages, which promoted the idea of immunological polarization. These states characterize macrophage proinflammatory vs anti-inflammatory properties, respectively (Martinez and Gordon 2014, Karampoor et al. 2021). On the other hand, it is wellfound that in vivo, macrophage activation occurs along with pro-inflammatory and anti-inflammatory conditions, with a degree of flexibility in both directions (Yamada and Kielian 2019). Immune cell polarization is widely detected in host responses related to inflammation, cancer, microbial immunity, fibrosis, and tissue regeneration (Ma 2020). Immune cells develop diverse programming and execute specialized roles in response to particular signals throughout this process (Ma 2020). This section will overview the polarization of immune responses in the context of cancer and biofilm infection.

\section{Immune polarization in cancer}

Innate immune cells such as macrophages, mast cells, dendritic cells, granulocytes, and natural killer cells are the front line of protection toward microbes and foreign causes (Table 2) (Johansson et al. 2008). In response to impaired tissue homeostasis, host tissue-resident innate immune cells regionally discharge soluble agents including chemokines, cytokines, matrix remodeling proteins, and other biologically active media that employ additional leukocytes from tissue circulation, i.e., inflammation (Johansson et al. 2008; Mirzaei et al. 2021c; 2021d). In response to a pathogen attack, specific recruited immune cells (also known as inflammatory cells) specifically kill pathogens in situ (Johansson et al. 2008). Instead, expert antigen-presenting cells (APCs) such as macrophages and dendritic cells, take foreign antigens such as tumor-associated antigens and move to lymphoid organ systems where they display their antigens to immune cells belong to the adaptive arm of the immune system (Johansson et al. 2008). Upon identification of the presented antigens, B lymphocytes, CD8+cytotoxic $\mathrm{T}$ lymphocytes (CTL), and CD4+ T helper (Th) lymphocytes mount an 'acquired immune response to foreign agents (Johansson et al. 2008, 2021). These processes paved the way for stimulating antigenically engaged adaptive immune responses by severe innate immunity stimulation (Johansson et al. 2008). Once foreign agents are removed, inflammation solves, and tissue homeostasis is reconstructed (Johansson et al. 2008). Given the risk of cancer progression, it is instantly apparent that related immunological reactions needed to activate severe inflammation may be co-opted so that if prolonged inflammation is retained in the tissue, it may instead facilitate the neoplastic programming of the tissue and promote the development of tumor (Fig. 2) (Coussens and Werb 2002; Balkwill et al. 2005).

\section{Macrophage}

Macrophages originate from the myeloid lineage and are part of innate immunity (Weagel et al. 2015). They are produced from blood monocytes that spread to tissues (Weagel et al. 2015). One of the key roles of macrophages is phagocytosis of bacteria and clearance of cellular debris (Weagel et al. 2015). They also contribute significantly to the induction and clearance of inflammation (Mantovani et al. 2013; Porta et al. 2009). Also, macrophages can show different reactions based on the type of stimulus they obtain from the underlying microenvironment, differing from pro-inflammatory to antiinflammatory (Sica and Mantovani 2012). In particular, two main macrophage phenotypes have been suggested: pro-inflammatory macrophages (M1 macrophages) and anti-inflammatory macrophages (M2 macrophages) corresponding to the phenotypes of a range of responses (Weagel et al. 2015).

M1 macrophages are defensive and strongly phagocytic, contain vast quantities of reactive oxygen and nitrogen species, and facilitate Th1 immune response (Sica and Mantovani 2012). This is a macrophage phenotype typically seen throughout infectious diseases. 


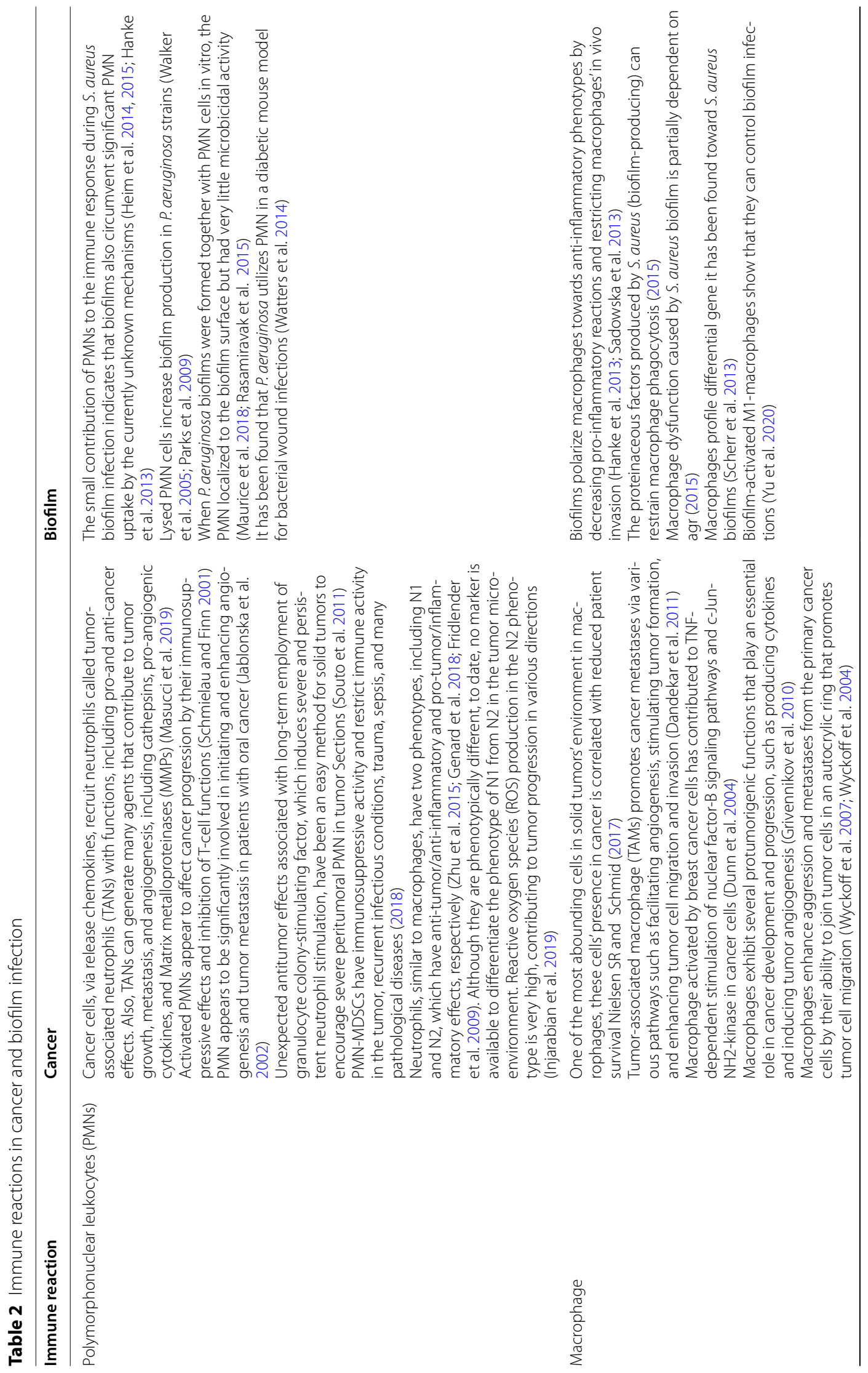




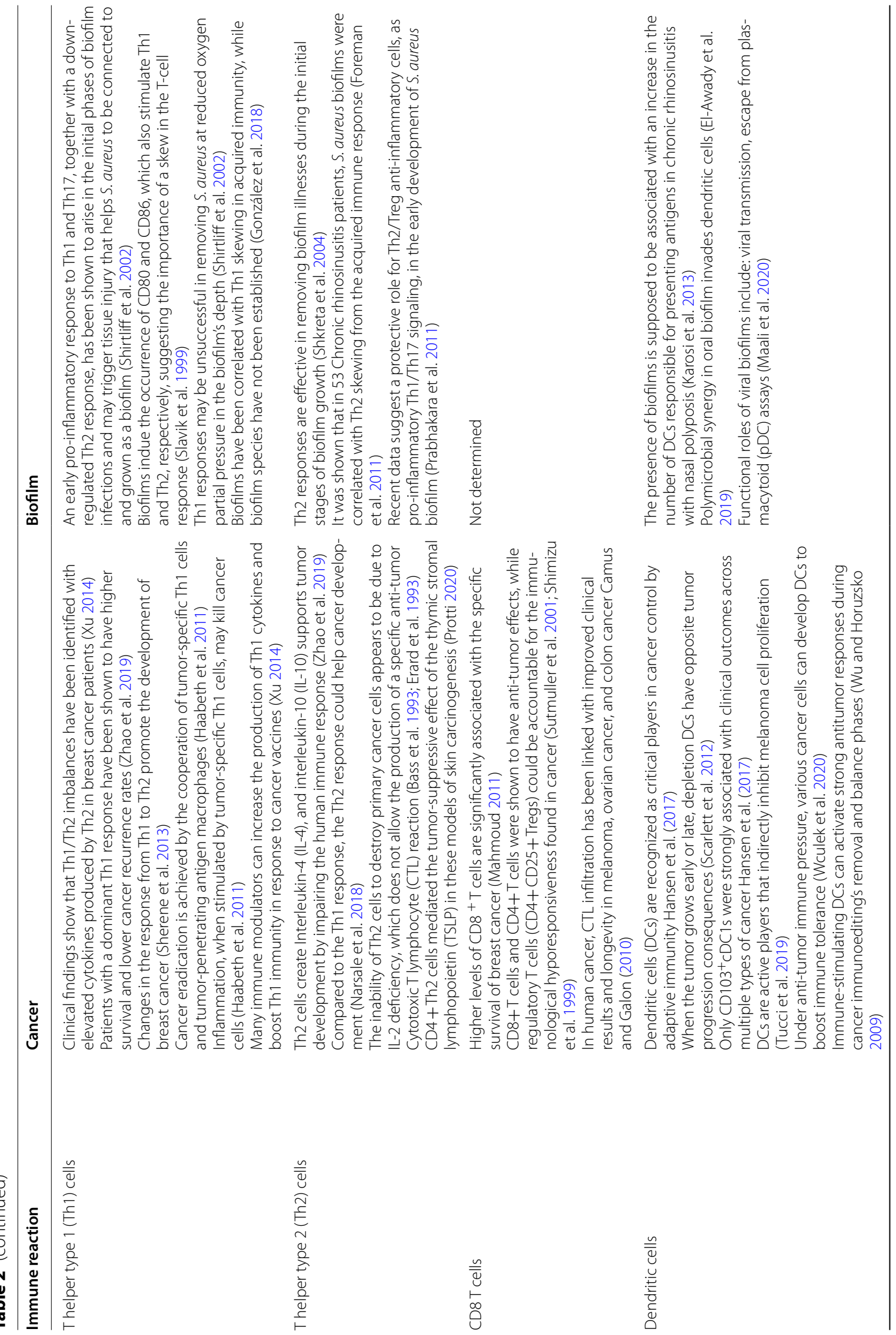




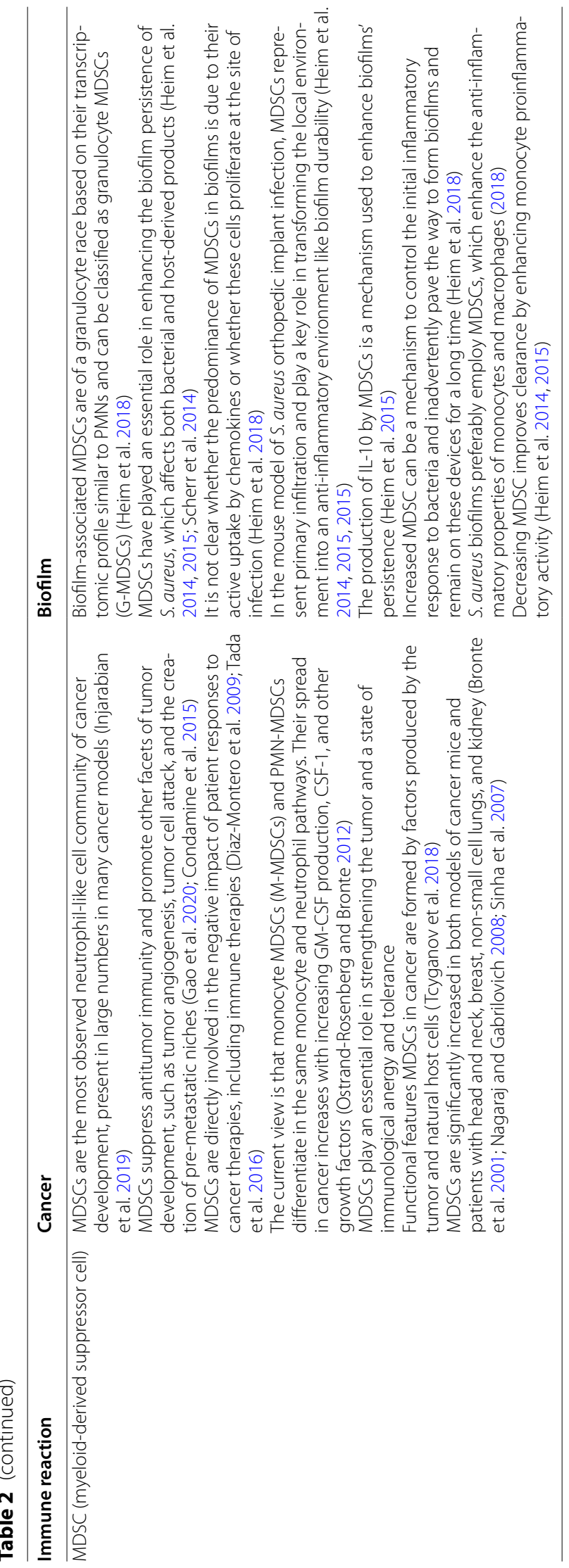




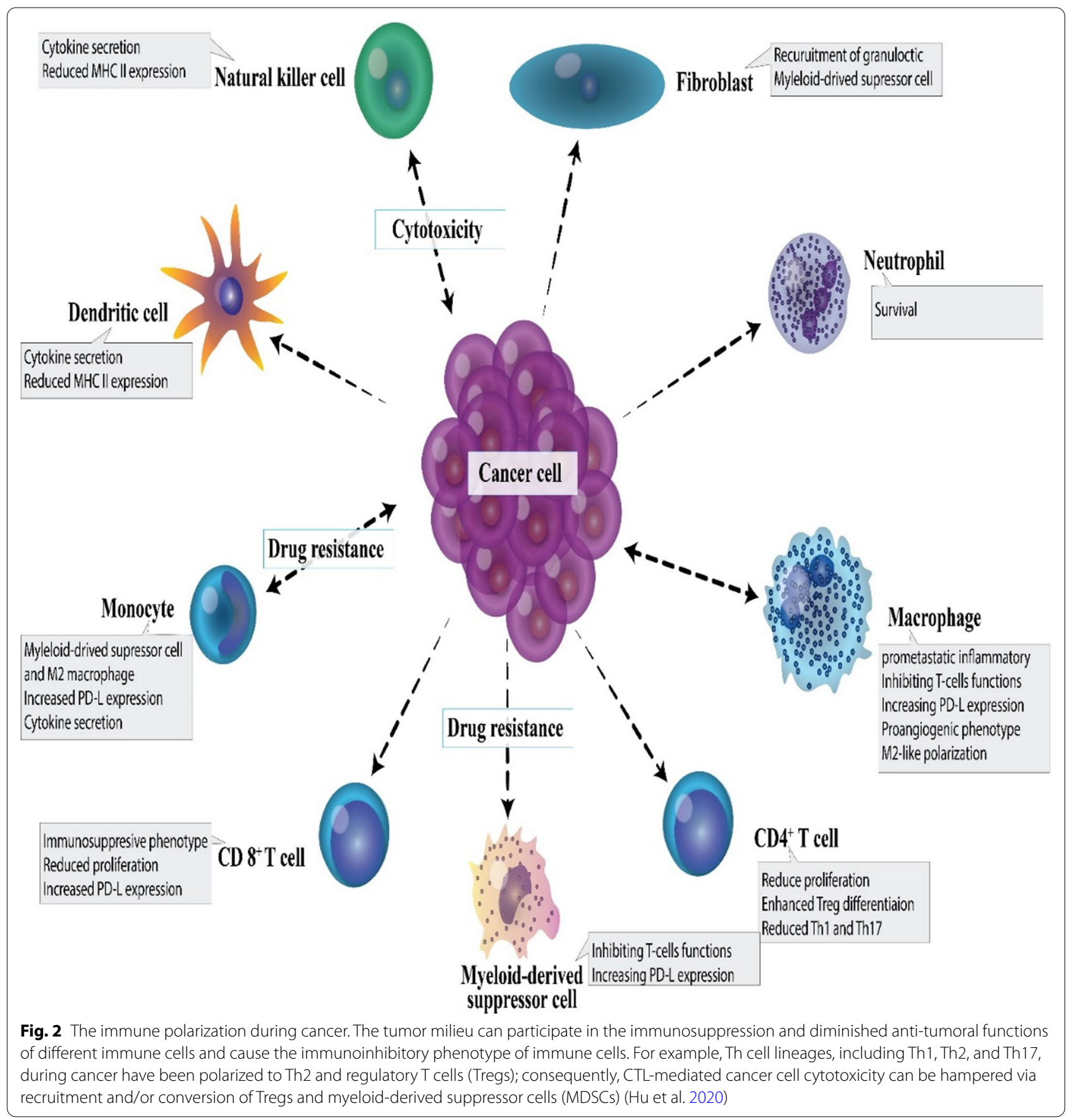

M1 macrophages produce elevated amounts of IL-12 and IL-23, two significant inflammatory cytokines. IL-12 causes induction and clonal expansion of Th17 cells, which secrete elevated levels of IL-17 and hence lead to inflammation (Hao et al. 2012). Succinate stabilizes HIF-1 $\alpha$, accelerating the transition to glycolysis and increasing inflammation. Specifically, it performs this by hindering the function of prolyl hydroxylase enzymes, so preventing them from hydroxylation and destabilizing
HIF-1 $\alpha$ (Kelly and O'Neill 2015). In this case, HIF-1 may interface with coactivators to trigger the glycolytic metabolic process and to promote inflammation by boosting transcription of the IL-1 $\beta$ gene (Tannahill et al. 2013).

M1 macrophages are thought to perform an essential role in detecting and removing cancer cells, and their involvement typically implies a favorable prognosis (Weagel et al. 2015). Following detection of tumor cells by macrophages, various pathways can kill tumor cells, including 
contact-dependent phagocytosis and cytotoxicity (i.e., activation of cytokine like TNF- $\alpha$ ) (Sinha et al. 2005). However, environmental stimuli such as tumor microenvironment or tissue-resident cells may polarize M1 to M2 macrophages (Weagel et al. 2015). The microenvironment of the tumor significantly impacts the differentiation of macrophages. The polarization mechanism can be dynamic and complex due to the great diversity in the rate of IL-10, apoptotic cells, glucocorticoid hormones, and immune complexes that may conflict with the role of innate immunity (Guiducci et al. 2005; Sica and Mantovani 2012). Notch signaling represents a vital function in the differentiation of M1 macrophages in the tumor microenvironment. It helps the transcription factor recombination signal binding protein for the immunoglobulin kappa J region (RBPJ) to control classical activation (Weagel et al. 2015). Macrophages defective in notch signaling transmit the M2 phenotype, independent of other extrinsic triggers (Wang et al. 2010). A range of stimuli give rise to the M2 phenotype, so it is essential to include the inducing molecule when contributing to these subtypes (Murray et al. 2014). The macrophages M2a are triggered by IL-13 and IL-4 (Wang et al. 2010). IL-4 and IL-13 bind to IL-4R and stimulate the JAK/ STAT6 cascade that controls the expression of Chemokine (C-C motif) ligand 17 (CCL17, ARG1 (Arginase 1), IRF4 (Interferon regulatory factor 4), IL-10, Cytokine signaling suppressor 3 (SOCS3), which are genes associated with anti-inflammatory reactions (Murray et al. 2014). M2b macrophages are generally trigered by $\mathrm{Fc}+\mathrm{TLR} / \mathrm{IL} 1-\mathrm{R}$ ligands (Wang et al. 2010). Besides, these macrophages are represented by IL-10, Chemokine ligand 1 (CCL1), Interleukin-1 (IL-1), and IL-6 (Özen et al. 2011). M2c macrophages are polarized in the presence of IL-10+TGF- $\beta$ (Özen et al. 2011). They control the development of IL-10 and TGF- $\beta$ and display CD163 and CD206 and many scavenger receptors (Lu et al. 2013; Chanmee et al. 2014).

\section{Tlymphocyte}

Premalignant and cancerous tissues are reported to be correlated with improvements in the leukocyte populations and their performance blocked CTL reactions which involve in tumor rejection, in conjunction with improved humoral immunity, can facilitate tumorigenesis (Tan and Coussens 2007; Colombo and Piconese 2007). Peculiar CD4+ T-cell subsets such as Th1, Th2, and Th17 cells, release distinct collections of cytokines that mediate their reactions (Tan and Coussens 2007; Colombo and Piconese 2007). For example, Th1 cells generate, Interleukin-2 (IL2 ) and Interferon- $\gamma($ IFN $-\gamma)$ and direct CTL reactions. In contrast, Th2 cells provide Interleukin-4 (IL-4), Interleukin-13 (IL-13), and Interleukin-10 (IL-10) and promote local humoral immune responses, while Th17 skewed cells generate Interleukin-23 (IL-23), IL-6, and tumor necrosis factor- $\alpha$ (TNF- $\alpha$ ), factors that reinforce and/or maintain persistent inflammation (Johansson et al. 2008). In peripheral blood of bladder and colon cancer cases, the balance of Th1 cells recognized by the intracellular increase of IFN- $\gamma$ or IL-2 is reduced. In contrast, the proportion of Th2 cells generated by IL-4, IL-6, and/or IL-10 is considerably higher than the contrarily healthy cohorts (Agarwal et al. 2006; Kanazawa et al. 2005). Recent research examining the properties of leukocytic infiltration in human cervical cancer reported that CD3+T-cell tumor infiltration shows heightened Th2 cytokine profiles and explicitly raised IL-4 and reduced IFN- $\gamma$ (Sheu et al. 2001). In line with these results, enhancements in immune cell status (removed CTL responses and developed humoral immunity) have also been documented in persistent inflammatory disorders correlated with developed cancer risk (Tan and Coussens 2007). Overall, these persuasive clinical decisions demonstrate that improved pro-tumor immune responses underlie an elevated likelihood of neoplastic development in tissues impacted by persistent inflammatory disease pathologies and/or tissues that harbor neoplasm-initiated cells.

The precise immune reactions to the cancer are driven by encounters with mature APCs and the existence of a pro-inflammatory environment (Dunn et al. 2002). In this sense, the regulatory $\mathrm{CD} 4+\mathrm{T}$ lymphocytes play a significant role in orchestrating reactions (Dunn et al. 2002). Naive $\mathrm{T}$ lymphocytes with the same antigen specificity can be 'polarized' into diverse functional effector cells that rely on the early environmental signal obtained when the antigen is displayed (Guy 2007). These signals are provided by innate immune cells and cells at the lesion site and are received by receptors displayed by naive T-lymphocyte precursors (Akira et al. 2006). For example, following viral infection, infected cells instantly discharge type I IFNs (IFN- $\alpha$ and/or $\beta$ ) that stimulate initial viral defense strategies and are also crucial for polarization of the immune system towards antiviral Th1 response (Levy et al. 2001; Proietti et al. 2002; Abdi and Mirzaei 2020).

Effector CD4+ T lymphocytes of Th1 progenitors have adapted to eliminate intracellular pathogens, including intracellular bacteria and viruses, by triggering CTL reactions and inducing IgG2a and IgG3 development (Weaver et al. 2006). In the sense of tumor metastasis, some IFNs have been hypothesized to mediate anti-tumor protection, in part by controlling divisive Th1 responses (Dunn et al. 2005). IFN-caused activation of Th1 cell differentiation when T-cell receptor (TCR) stimulation is followed by IFN-induced signaling via the signal transducer and STAT1 intracellular signaling cascade, the pioneer stage of Th1 differentiation (Lighvani et al. 2001). Signaling by STAT1 upregulates the formation of the $R \beta 2$ chain of the Interleukin-12 (IL-12) receptor, thereby making cells 
receptive to IL-12, a cytokine essential for further differentiation of the Th1 cells (Szabo et al. 1995). This mechanism also inhibits the development of IFN- $\gamma$ and provokes the expression of Interleukin-18 (IL-18) R $\alpha$ receptor (Dinarello 1999). Mature Th1 effector cells may generate IFN- $\gamma$ via TCR-dependent mechanisms and generate cytokines independently of antigen stimulation while being triggered by IL-12 and IL-18 (Yang et al. 1999).

Th2 lineage cells are believed to have developed to strengthen the removal of infectious diseases and are distinguished by the development of IL-4, IL-13, and Interleukin-5 (IL-5) (Lee et al. 2001). These cytokines are useful actuators of immunoglobulin E (IgE) development and recruiting of eosinophils and granulocytes (Lee et al. 2001). The configuration of Th2 cells is triggered by TCR signaling and IL-4 signaling via STAT6, occurring by epigenetic chromatin rearrangement in the Th2 cytokine bunch while concurrently restricting STAT4 and IL-12 $\mathrm{R} \beta 2$ expression (Reiner 2005; Ouyang et al. 2000). Together, these activities support the production of the Th2-related cytokines, making them resistant to the Th1 linear engagement's repolarization.

Recent findings have shown a greater variety in the CD4+T-cell effector range and have related the cytokines IL-17 and IL-23 to a new branch of the Thcell family known as Th17 cells (Mirzaei et al. 2021e; Cua et al. 2003). The production of Th17 cells from naive $\mathrm{T}$ cells is caused by the transformation of the growth factor- $\beta$ (TGF- $\beta$ ) and IL- 6 as an essential cofactor (Bettelli et al. 2006). TCR activation can directly induce the development of Th17 cytokines, whereas IL-1, IL-23, and IL-18 can potentiate this effect (Weaver et al. 2006). CD4+ Th17 cells have been active in removing extracellular bacteria and autoimmune diseases, such as experimental autoimmune encephalomyelitis (EAE) and collagen-induced arthritis. They are correlated with granulocyte recruitment and expression of Immunoglobulin M (IgM), IgG, and Immunoglobulin A (IgA) (Weaver et al. 2006; Annunziato et al. 2007). The Th17 responses were also related to facets of cancer growth (Langowski et al. 2006). In an in vivo model of chemically mediated skin carcinogenesis, IL-23 was considered as an essential inflammatory response mediator correlated with cancer advancement (Langowski et al. 2006).

The fourth type of CD4+ cells, called regulatory $\mathrm{T}$ (Treg) cells, inhibits the effector action of CTLs and performs essential physiological functions in preventing autoimmune disorder, and intensifies immunity from infections (Zou 2005; Colombo and Piconese 2007, 2020). Treg cells have been defined as normal Treg cells that develop in the thymus, and adaptive Treg cells that are distinguished in peripheral tissues (Karampoor et al. 2020). Natural Treg cells are CD4+CD25+Forkhead box protein
P3 (FOXP3) + and shape predominantly in the thymus through high-affinity TCR with antigens expressed in the thymic stroma (Colombo and Piconese 2007; Fehérvari and Sakaguchi 2004; Weaver et al. 2006). Natural Treg cells repress immune reactions by cell covering molecules such as cytotoxic T-lymphocyte antigen 4 (CTLA4), membrane-bound TGF- $\beta$, and pericellular adenosine production (Colombo and Piconese 2007; Fehérvari and Sakaguchi 2004; Weaver et al. 2006). Adaptive Treg cells are $\mathrm{CD} 4+\mathrm{CD} 25+$ FoxP3+/low, developed in peripheral tissues in the presence of TGF- $\beta$ and IL-10, which inhibit immune responses primarily by releasing soluble factors, including IL-10 and TGF- $\beta$ (Weaver et al. 2006). In vivo Treg cell loss employing CD25 neutralizing antibodies augments the T-cell anti-tumor reaction and causes relapse of laboratory cancers, such as sarcomas and melanomas (Onizuka et al. 1999, 1999). Clinical reports showed that the presence of Treg cells in patients with ovarian cancer is associated with reduced survival and these results can suggest the essential function of Treg cells in controlling proand anti-tumor immunity (Curiel et al. 2004).

\section{Immune polarization in biofilm infection}

In chronic biofilm-associated device infections, internalized bacteria and cells in biofilms could adopt a phenotype of small colony variant (SCV), which is known for a slow rate of growth and reduced levels of secretion of toxic factors, which enable the internalized bacterial cells to survive for long periods (Bui et al. 2017; Proctor et al. 2006). The lack of diffusion of leukocytes initially described resistance against leukocytes within the biofilm and a decreased capacity of PMNs to kill the bacteria embedded in biofilm (Fig. 3) (Arciola et al. 2018). The mature biofilms have dense exo-polymeric substances that are difficult to engulf by macrophages. This leads to neutralized phagocytosis, a phenomenon that has been proposed primarily to explain the reaction of phagocytes to asbestos fibers (Thurlow et al. 2011; Donaldson et al. 2010). In vivo investigations have found that Staphylococci biofilm can deviate the innate immune responses to the pro-fibrotic and anti-inflammatory reactions, rather than the pro-inflammatory, and bactericidal reactions (Arciola et al. 2018, Hanke et al. 2012). The bacterial biofilm directs macrophage polarization from the classic pro-inflammatory phenotype to the anti-inflammatory form, the latter is characterized by the formation of antiinflammatory mediators (Gries and Kielian 2017; Hanke and Kielian 2012). In device-associated biofilm infections, IL-12 triggers the recruitment of myeloid suppressor immune cells, which mediate the anti-inflammatory effects via their strong immunosuppressive ability and impairing phagocyte influx (Heim et al. 2015). 


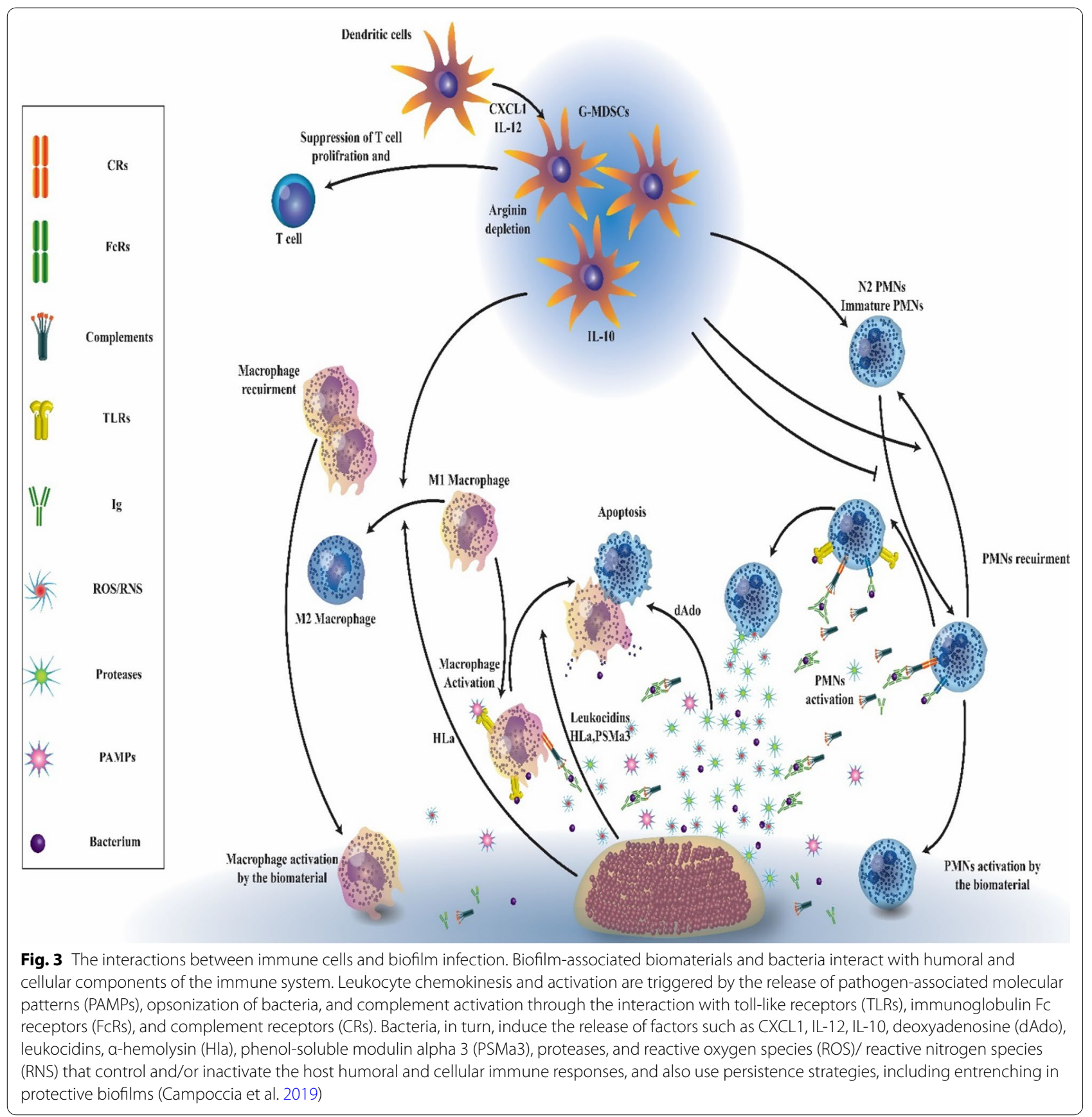

\section{Polymorphonuclear leukocytes (PMNs)}

The human immune system not only responds to the bacterial cells that contaminate medical devices, but it also responds to the biomaterial surface of the devices and senses them as the foreign body. This response stimulates inflammatory reactions that contribute to the complement system, coagulation cascade, immune cells, platelets, and particularly PMNs (Franz et al. 2011). The activation of PMNs triggers metabolic weakness and discharge of oxidative resources, resulting in the permanent release of Reactive oxygen species (ROS), which diminishes their killing ability to kill the bacterial cells (Arciola et al. 2018). The reduced bactericidal ability of PMN following exposure to the various surfaces has been demonstrated in severe biofilm-associated bacterial infections (Arciola et al. 2018). Besides the exhaustion of immune reactions due to the presence of medical devices, the bacterial cells apply various methods to evade human 
immunity, e.g., invasion of human cells, skewing of the immune reactions, and toxin production (Arciola et al. 2018). While many of the previous studies evaluating the bactericidal capacity of PMN were performed using planktonic cultures of bacterial cells, there has been a wave of recent works examining the interaction between bacterial biofilm and PMN. Most studies of the interactions between biofilm and PMNs were reported with the biofilms of Staphylococcus aureus and Pseudomonas aeruginosa (Alves et al. 2018).

When $P$. aeruginosa biofilms were formed together with PMN cells in vitro, the PMN localized to the biofilm surface but had very little microbicidal activity (Rasamiravaka et al. 2015a; Maurice et al. 2018). One reason for this phenomenon is that alginate polysaccharide present in the biofilm EPS matrix of $P$ aeruginosa suppresses phagocytosis and chemotaxis of PMN (Maurice et al. 2018). Rhamnolipid is another secreted component produced in $P$. aeruginosa biofilms, which antagonizes PMN cells (Jensen et al. 2010). Jensen et al. (2007) found that rhamnolipid formed by $P$. aeruginosa biofilm quickly lysed host PMNs in vitro. They showed that $P$. aeruginosa strains upregulated rhamnolipid production in reaction to the PMN exposure, indicating that $P$. aeruginosa actively senses and reacts to these immune cells (Jensen et al. 2007). Many reports show that lysed PMN cells increase biofilm production in $P$. aeruginosa strains (Parks et al. 2009; Walker et al. 2005). For example, PMN destroyed the planktonic $P$. aeruginosa strains, while biofilms increased survival (Walker et al. 2005). P. aeruginosa was found to use actin and DNA from the lysate of PMN to reinforce its biofilm (Gennip et al. 2012). In addition, the PMN-increased biofilm of $P$. aeruginosa was somewhat tolerant to antimicrobials. When the biofilm was exposed to DNase, it was destroyed and planktonic cells were dispersed (Walker et al. 2005). Besides, the ability of $P$. aeruginosa to benefit from PMN was recently reported in a diabetic mouse model for wound bacterial infections (Watters et al. 2014). Diabetic mice on insulin treatment were wounded and inoculated with $P$. aeruginosa, and it was demonstrated that PMNs had enhanced migration compared to the non-diabetic mouse. Nevertheless, this enhanced recruitment did not lead to enhanced clearance of bacteria. The increased migration of PMN in this mouse model resulted in the formation of $P$. aeruginosa biofilms that were enriched with DNA and showed enhanced tolerance to the aminoglycoside antibiotic gentamicin (Watters et al. 2014).

Further studies presented the disadvantageous interactions between PMNs and biofilm. In a study by Nguyen et al. (Nguyen et al. 2013), a T2 (type 2) diabetic mouse model of wound infection was used to demonstrate that the existence of $S$. aureus biofilm significantly diminished the oxidative activity of PMN, triggering a higher bacterial load and reduced rates of healing. While $S$. aureus stimulated the Neutrophil extracellular trap (NET)osis of PMN cells, it was also shown that $S$. aureus secretes enzymes that produce deoxyadenosine from NET degradation, which then stimulates the caspase 3 (Cas 3) mediated death of immune cells (Watters et al. 2015).

Additionally, clinical infections of Staphylococci are associated with diminished apoptosis of PMNs in diabetic persons, which direct the prolonged formation of TNF- $\alpha$ and thus reduce the bacterial clearance of PMNs (Hanses et al. 2011; Mirzaei et al. 2017; Mirzaei et al. 2020e; Rasoul et al. 2019). PMNs are impressive at killing planktonic bacterial cells; however, bacterial biofilm emerges to resist the microbicidal activities of PMNs, and can even profit from the cellular debris left behind the matrix. It is evident from these works that the interplay between biofilm and the dying and ineffective PMN contributes powerfully to the chronic inflammatory mode present in chronic bacterial infections (Watters et al. 2015). PMNs can be considered predator cells and bacterial pathogens as prey; however, the outcome does not always favor the predators, as seen with the interaction between PMNs and Staphylococcus biofilms. The PMN cells attack the bacteria directly and the EPS slime layer (Hänsch et al. 2012). The EPS structure and composition differ broadly among the bacterial species, and even within a single species, there is variation between strains. For example, the mucoid $P$. aeruginosa strains collected from Cystic fibrosis (CF) patients produced the alginate polysaccharide as the main EPS component, whereas non-mucoid strains did not (Jesaitis et al. 2003). Therefore, when considering the interaction between PMNs and EPS biofilm, the outcomes cannot be generalized to all pathogens. Applying the experimental findings from various animal models to human bacterial infections is relatively challenging because they do not fully represent natural human infections. For example, mice produce fewer PMNs than humans (approximately 20\% of the leukocytes); hence it is feasible that the initial innate immune reactions in animals may not resemble the immune responses in humans.

\section{Macrophage}

Much research has focused on PMNs, but it has been shown that monocytes share many functional properties with PMNs (Hänsch et al. 2012; Jesaitis et al. 2003). Following the recruitment of PMNs to bacterial infection sites, macrophages arrive a few days later in response to the damage from infections that were not resolved by PMNs (Watters et al. 2015). The main function macrophages, namely efferocytosis, is to phagocytize the 
apoptotic and necrotic host cells (Khanna et al. 2010). Other macrophage functions include the formation of ROS to destroy bacteria during phagocytosis. However, the interplay of macrophages with bacterial biofilm is less well-studied than that of neutrophils. In recent years, it was shown that a quorum sensing (QS) molecule, $\mathrm{N}$-acyl homoserine lactone (AHL) produced by $P$. aeruginosa, stimulated apoptosis in macrophages (Tateda et al. 2003).

Nevertheless, AHL molecules have also been demonstrated to stimulate phagocytosis in macrophages while not impacting ROS production (Vikström et al. 2005). In a work when biofilm was co-cultured with macrophages, a $P$. aeruginosa strain lacking the alginate polysaccharide was destroyed by macrophages in a few hours (Leid et al. 2005). The killing of this biofilm was attributed to phagocytosis and was dependent on IFN- $\gamma$.

Several biofilm evasion strategies have been found in the innate immunity including reductions in TNF- $\alpha$, IL-1 beta (IL-1 $\beta$ ), C-X-C Motif Chemokine Ligand 2 (CXCL2), and C-C Motif Chemokine Ligand 2 (CCL2) formation that limited macrophage invasion into the biofilm. Also, there was a skewing of the immune cells away from a bactericidal phenotype, as determined by a decrease in the formation of inducible nitric oxide synthase (iNOS) concomitant with robust ARG-1 induction (Campoccia et al. 2019, 2015; Le et al. 2018). Interactions of macrophages with biofilm show limited phagocytosis ability as seen in M2 macrophages (Campoccia et al. 2019; Thurlow et al. 2011). Although PMN is a critical anti-bacterial effector, its transcriptional ability to produce inflammatory cytokines is restricted, and because of its short life term and high cell turnover, it needs constant PMN recruitment (Campoccia et al. 2019; Yamada and Kielian 2019). By contrast, macrophages are more long-lived, produce different rates of proinflammatory molecules are important for recruitment/activation cascades of immune cells after bacterial exposure, and show potent anti-bacterial and phagocytic impacts (Campoccia et al. 2019; Yamada and Kielian 2019). Furthermore, resident macrophages are present in almost all tissues and serve as a critical primary defense mechanism against bacterial invasion by recognition via Toll-like receptors (TLRs) (Hirayama et al. 2017).

$S$. aureus biofilm triggers macrophage dysfunction in many aspects, which provide a full explanation as of why biofilm infections persist in immunocompetent individuals (Alboslemy et al. 2019; Scherr et al. 2015; Thurlow et al. 2011). However, the bacterial biofilm stimulates the influx of higher macrophages in early infection; the lack of iNOS and the massive production of ARG-1 show that bacterial cells may not be completely eradicated (Yamada and Kielian 2019;
Thurlow et al. 2011). The same M2-like phenotypes were found in macrophages following biofilm co-culture (Yamada and Kielian 2019; Thurlow et al. 2011). In the second place, although biofilm was mediated to a vast accumulation of macrophages, only a few subsets migrated to the surface of the biofilm, and most produce ARG-1, which skews macrophages for ineffective killing. Latter, the data found that biofilm triggers macrophages' death by an unknown process. Notably, macrophages were capable of phagocytizing bacterial cells from the dispersed biofilm, showing that the size and inability of opsonizing intact biofilm can describe the phenotype of the dystrophic macrophages. The process that induces macrophages' death following contact with the biofilm is not well defined but can be the consequence of bacterial toxins, acidic $\mathrm{pH}$, and the hypoxic microenvironment surrounding the biofilm (Lebeaux et al. 2014; Koo et al. 2017). However, findings collectively show that the biofilm re-programs macrophages responsiveness to an M2 phenotype with less anti-bacterial ability. All in all, it is proposed that biofilm attenuates the formation of inflammatory mediators and the invasion of macrophages to the site of infection. Also, the production of iNOS was mitigated, and a coincident rise in ARG-1 collectively demonstrates a skewing of the immune reactions to a pro-fibrotic M2 phenotype.

Nosocomial infection (like medical device infection) is a costly problem for the health care system, and strains of $S$. aureus (in particular methicillin-resistant $S$. aureus) are the predominant species that cause this infection (Haque et al. 2018, 2012). The medical device infection is associated with the ability of $S$. aureus to attach to the host coating devices by host protein via cell wall bacterial adhesins. Biofilm of $S$. aureus makes eradication of the infection difficult because of increased resistance of the embedded bacterial cell to antibiotics and immune reactions (Hanke et al. 2012). Some studies have found an effect on IL-1 $\beta$ in biofilm infection, whereas TLRs had no influence (Hanke et al. 2012). Interestingly, both IL-1 receptors (IL-1R) and TLRs are involved in signaling pathways related to Myeloid differentiation primary response 88 (MyD88). However, the role of this crucial factor in modulating response to biofilm infection is unclear (Hanke et al. 2012). A study by Mark et al. (2012) evaluated the biofilm infection by $S$. aureus in MyD88 knockout mice. In the study, MyD88 knockout mice showed more bacterial infection compared to wild-type mice. Production of inflammatory mediators such as IFN-c, IL-6, and Chemokine $\mathrm{C}-\mathrm{X}-\mathrm{C}$ motif ligand 1 (CXCL1) was significantly reduced in MyD88 knockout animals. Tissue staining for biofilm by the immunofluorescence 
technique showed an increased fibrosis in these mice, coinciding with increased recruitment of M2 macrophages. According to previous works on TLR2, TLR9, and IL-1 $\beta$ knockout mice, the report of Market al. (2012) suggests that MyD88 signaling is a practical pathway in regulating fibrosis and the polarization of macrophages in biofilm formation. In sum, the current data indicate that the absence of MyD88 (not "signaling mediated to MyD88") exacerbates the polarization of M2 and fibrosis in the biofilm infection of $S$. aureus, resulting in the growth and propagation of bacteria during acute infection. Taken in the context of previous studies with TLR2 and TLR9 knockout animals, where these indices were not affected, the recent studies can enhance our understanding of MyD88-dependent cascades, indicating the production of inflammatory mediators that do not involve TLRs (Hanke et al. 2012). Therefore, we note that new approaches to disrupt the signaling process mediated by MyD88 could be effective in the treatment of biofilm infection.

\section{MDSC}

MDSC is a subset of immature granulocytes and monocytes that are intermediates of common myeloid differentiation and development (Heim et al. 2015; Gabrilovich and Nagaraj 2009). In a normal situation, MDSCs differentiate at the location of inflammation to form mature myeloid populations, including dendritic cells, PMN, as well as macrophages (Gabrilovich and Nagaraj 2009; Dai et al. 2015). However, under pathological conditions, like chronic inflammation, tumors, and bacterial biofilm-associated infections, MDSC is arrested in an immature mode, regulating inflammatory mechanisms by its repressive actions (Youn et al. 2012; Ostrand-Rosenberg and Sinha 2009). MDSC proliferation in cancer is caused by various growth factors and cytokines, like Granulocytemacrophage colony-stimulating factor (GM-CSF), IL-6, vascular endothelial growth factor (VEGF), and Granulocyte-colony stimulating factor (G-CSF) (Ostrand-Rosenberg and Sinha 2009). Nevertheless, it is unclear whether the host or bacterial components release MDSC in the biofilm infection or arrest it in its immature state.

Following the development of MDSCs, inflammatory stimuli generate and cause immunosuppressive effects (Condamine and Gabrilovich 2011). IL-12 has been shown to facilitate MDSC recruitment in the $S$. aureus biofilm, which may be an indirect impact, as this cytokine is not a chemo-attractant agent (Heim et al. 2015). Nevertheless, IL-12 cytokine is not required for the activation of MDSCs in the biofilm infection since
MDSCs from both p35 knockout, and IL-12 p40 mice still inhibit the proliferation of CD4+ T cells (Heim et al. 2015). Hence, other inflammatory agents should contribute to the production of IL-10, ARG-1, and other anti-inflammatory components formed by MDSCs with immunosuppressive activities against the $S$. aureus biofilm (Heim et al. 2015). IL-10 is a well-defined antiinflammatory cytokine that controls inflammatory reactions, e.g., suppressing the activation and polarization of $\mathrm{T}$ cells and production of IL-10 by MDSCs promotes the polarization macrophages to the anti-inflammatory state (Bunt et al. 2009; Murray 2005, 1993). MDSCs are crucial in the anti-inflammatory reactions against $S$. aureus strains and enhance the chronic infection (Heim et al. 2014). Biofilm-associated infection is known to hijack the innate immune response towards an anti-inflammatory mode (Fig. 3) (Heim et al. 2014). In this regard, IL-10 can facilitate the development of persistent infections and subvert mechanisms of immune eradication (Heim et al. 2014). Although the production of IL-10 by MDSCs has been implicated as an immunosuppressive mechanism, so far, there is no report demonstrating that the activity of MDSCs is mediated to IL-10 in biofilm infection. A study by Heim et al. (2014) found that MDSCs produce massive levels of IL-10 against the biofilm mode of growth, which restricts proinflammatory gene expression of monocyte and is directly involved in the biofilm persistence of $S$. aureus in later phases of infection. Although there are few reports of T-reg cells involved in the $S$. aureus biofilm, the production of IL-10 by MDSCs caused stimulation of T-reg cells that could generate IL-10, which may perpetuate the anti-inflammatory state (Heim et al. 2014). In future works, the mechanism of accumulation, proliferation, and activity of MDSCs in the biofilm infection must be examined.

Depending on the role of IL-10 in biofilm infections, $S$. aureus components that increased IL-10 synthesis by MDSCs and macrophages were discovered, such as genes that are involved in lactate synthesis, implying that bacterial lactate is an important determining factor of leukocyte activation (Heim et al. 2020). Lactate from $S$. aureus biofilms serves as a metabolic virulence factor, increasing IL-10 release in MDSCs and macrophages via blocking Histone deacetylase (HDAC). This reduces leukocyte pro-inflammatory function, which is one mechanism that accounts for biofilm persistence. This is similar to tumor cells generating lactate in the tumor microenvironment, promoting the development of MDSCs and immunosuppression. A study conducted by Husain et al. (Husain et al. 2013) discovered that increased lactate synthesis by tumor cells due to high glycolytic metabolism inhibited host immunological response to tumor cells through modulation of MDSCs function. 
Immunometabolism in cancer and biofilm infection Cancer

Metabolic reprogramming of Tumor-associated macrophages (TAMs), DCs, and $\mathrm{T}$ cells and their altered functions during tumor progression are dipticated in Figs. 4, 5, 6. In this section, we overview the immuneometabolism reactions in some immune cells during cancer (Table 3).

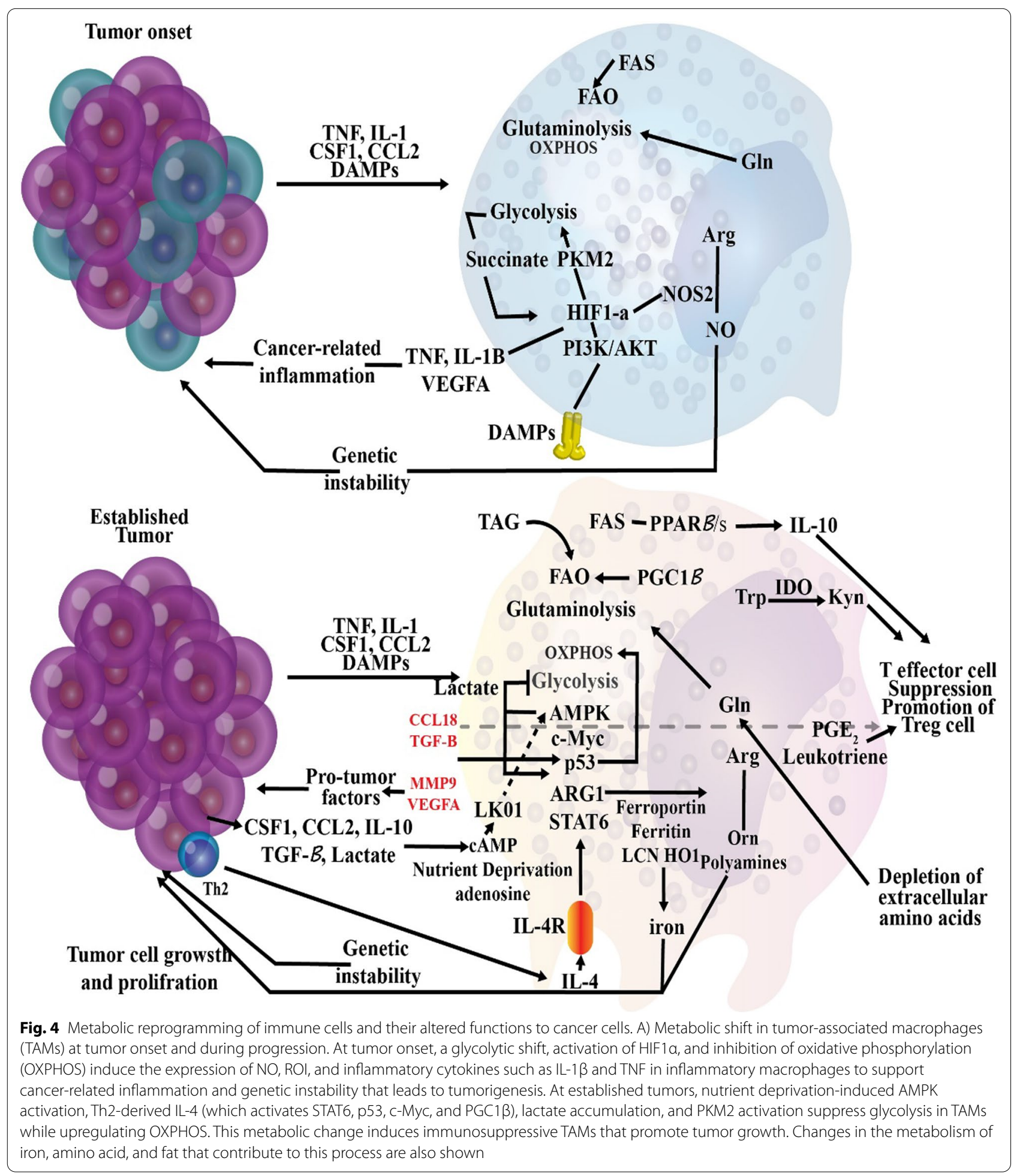




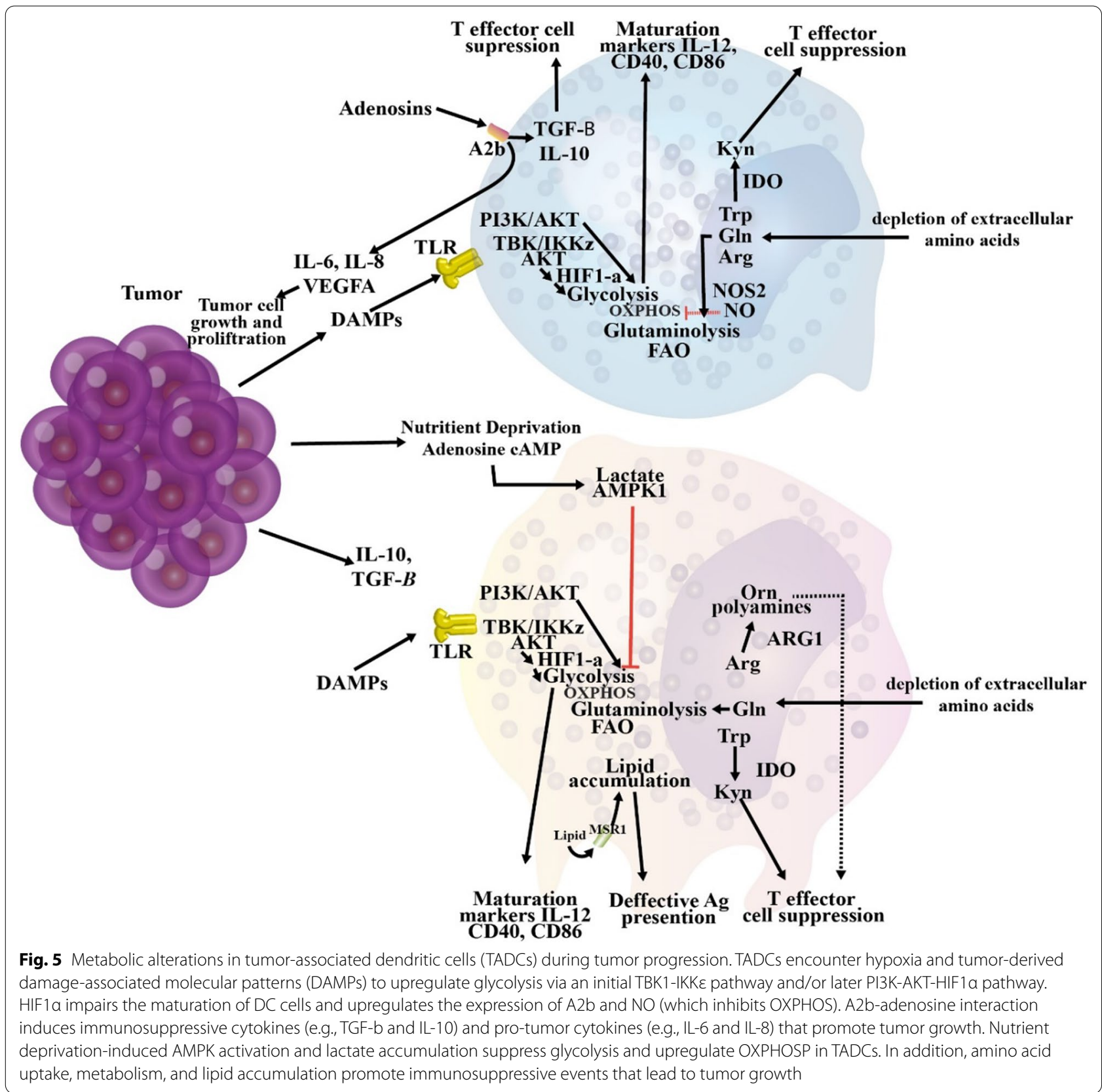

\section{T cells}

Immune cells such as stimulated cytotoxic T cells experience complete metabolic changes to conduct effector roles, such as destroying tumor cells and secreting cytokines (Table 2) (Singer et al. 2018). Aerobic glycolysis, a mechanism in which glucose is metabolized to pyruvate and eventually to lactate in a sequence of enzymatic reactions that generate ATP and substrates for other metabolic processes, was first described in cancerous cells (Singer et al. 2018). Intriguingly, nonmalignant proliferating cells undergo aerobic glycolysis, which is also deemed critical for optimum T-cell activity (Cham et al. 2008). Nevertheless, T cells are metabolically versatile, and aerobic glycolysis can not be essential to their stimulation and preservation (Singer et al. 2018; Cham et al. 2008). On the other hand, Glycolysis is vital for T-cell replication (Renner et al. 2015). 


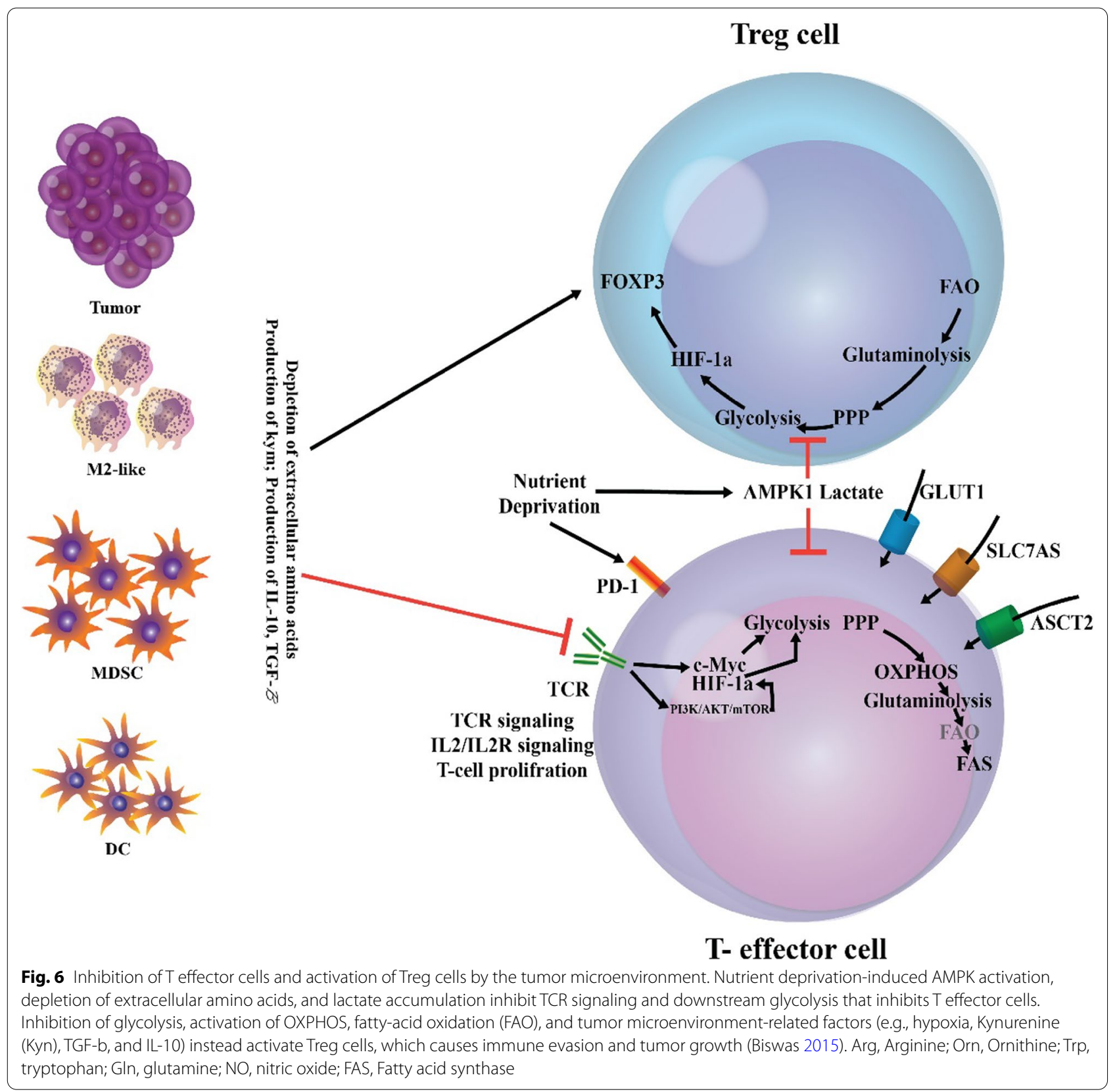

Under normoglycemic circumstances, $\mathrm{T}$ cells upregulate the main glucose converter Glucose transport protein type 1 (GLUT1), accompanied by enhanced glucose absorption and glycolysis following antigenic stimulation (Frauwirth et al. 2002; Macintyre et al. 2014). Also, pyruvate, one of the final metabolites of glycolysis, is primarily reduced to lactate rather than oxidized in mitochondrial respiration (Singer et al. 2018). However, T cells might not even be able to preserve a persistent status of the glycolytic pathway that is essential for their effector role (Singer et al. 2018). Aerobic glycolytic pathway contracts and memory $\mathrm{T}$ cells, which are less reliant on glycolysis and more participate in mitochondrial respiration, occur throughout acute infections (Buck et al. 2015; MacIver et al. 2013). On the other side, cancer cells can sustain and ultimately improve elevated glucose absorption and glycolysis, reducing intratumoral glucose levels (Voelxen et al. 2018; Battista et al. 2016). 
In contrast, glucose deficiency will directly inhibit the production of IFN- $\gamma$, a crucial T-cell effector molecule in CD8+ $\mathrm{T}$ cell tumor infiltration (Chang et al. 2015). It has been suggested that these results be facilitated by glyceraldehyde-3-phosphate dehydrogenase (GAPDH), a crucial anaerobic glycolysis enzyme that may also influence post-transcriptional alteration of mRNAs (Singer et al. 2018). When T lymphocytes perform high glycolytic pathway speeds, GAPDH is dedicated to their metabolic function (Singer et al. 2018). However, in the sense of lower glycolytic flux, GAPDH inhibits the translation of IFN- $\gamma$ (Chang et al. 2013). It has also been reported that glucose deficiency inhibits TCR-dependent $\mathrm{Ca} 2+$ Nuclear factor of activated $\mathrm{T}$ cells (NFAT) signaling, contributing to T-cell hyporesponsiveness (Singer et al. 2018). CD4+ Treg cells can suppress inflammation, often correlate with cancer development, and impede local anti-tumor defense (Singer et al. 2018). Of note, as demonstrated in murine models, Treg cells produce low rates of GLUT1, are not depend on glucose absorption and glycogenesis, and, like to CD8+ memory $\mathrm{T}$ cells, conduct OxPhos and lipid oxidation (Michalek et al. 2011). FOXP3, the lineage-defining transcription factor for murine Tregs, was suggested as the primary regulator of this phenotype (Gerriets et al. 2016). Mechanically, FOXP3 stimulates the development of genes which are required for synthesis synthesize of lipids and peptide hormones (Singer et al. 2018). It also decreases the control of genes involved in glucose absorption and glycolysis (Singer et al. 2018). Notably, the forced expression of FOXP3 prevents the phosphatidylinositol 3-kinase $(\mathrm{PI} 3 \mathrm{~K}) /$ protein kinase $\mathrm{B}(\mathrm{AKT})$ signaling cascade of Mammalian target of rapamycin complex 1 (mTORC1) implicated in the activation of glycolytic machines (Gerriets et al. 2016). Contradictory, glucose excess may be necessary for Treg initiation, as glycolysis in traditional CD4+ T cells is required for the induction of a regulatory phenotype in Treg cells by translocation of the glycolytic enzyme enolase-1 (ENO1) to the nucleus

Table 3 Metabolic changes in immune cells during cancer and biofilm infection

\begin{tabular}{|c|c|c|}
\hline Cell & Metabolic reactions in tumor & Metabolic reactions in biofilm \\
\hline Neutrophils & $\begin{array}{l}\text { Neutrophil metabolism is poorly defined in the sense of cancer, and the metabo- } \\
\text { lism of neutrophil-specific subgroups (i.e., N1 or N2) is understudied. In the sense } \\
\text { of inflammation, neutrophils need an improved flow of glycolysis and pentose } \\
\text { phosphate metabolism (PPP) to support nucleotide synthesis, respiratory bursting, } \\
\text { and Neutrophil extracellular trap (NET) development, with minimal criteria for the } \\
\text { tricarboxylic acid (TCA) cycle, oxidative phosphorylation (OXPHOS), and fatty acid } \\
\text { synthesis (FAS) } \\
\text { Glucose absorption, glycolysis, and metabolic change to PPP are significant for neu- } \\
\text { trophil netting sequesters circulating tumor cells and promoting metastases. The } \\
\text { data provide some suggestions about how the metabolic shift in neutrophils by } \\
\text { controlling functions such as NETs can lead to cancer development (Biswas 2015) } \\
\text { Together, tumor development and the acquisition of immunosuppressive neu- } \\
\text { trophils are concurrent with a complex development of their metabolism and } \\
\text { nutrient utilization, spanning from glucose-fueled glycolysis and OXPHOS to fatty } \\
\text { acid-fueled Fatty acid oxidation (FAO) in the initial and end stages, respectively } \\
\text { (Riera-Domingo et al. 2020) }\end{array}$ & $\begin{array}{l}\text { It has been found this diminished hypoxic levels } \\
\text { of oxygen result in diminishing neutrophil killing } \\
\text { ability and because this ability is mediated by the } \\
\text { formation of reactive oxygen species (ROS) from } \\
\text { oxygen (Jesaitis et al. 2003, 2018; Mandell 1974). } \\
\text { Also, suppose this hypoxic situation is continued. } \\
\text { In that case, neutrophils will be impaired in pro- } \\
\text { ducing ROS like hypochlorous acid and hydrogen } \\
\text { peroxide, causing impairment of the neutrophil's } \\
\text { ability to eliminate and control biofilm infection } \\
\text { (Wu et al. 2018) } \\
\text { PMNs in the cystic fibrosis (CF) airway of patients } \\
\text { has increased the generation of specific metabolic } \\
\text { and stress pathways such as CD39, CD114, C-X-C } \\
\text { motif chemokine receptor } 1 \text { (CXCR4), as well as } \\
\text { Receptor for Advanced Glycation Endproducts } \\
\text { (RAGE) (Makam et al. } 2009 \text { ). In this regard, it has } \\
\text { been noted that a shift in the formation of their } \\
\text { nutrient transporters influences the inorganic } \\
\text { phosphate and glucose transports that are part } \\
\text { of their adaptation to the airway of CF patients } \\
\text { (2013) } \\
\text { Of note, about of anaerobic situation in the mucus } \\
\text { of CF patients, Kolpen et al. (Kolpen et al. 2010) } \\
\text { hypothesized that the respiratory burst of the } \\
\text { polymorphonuclear leukocytes (PMNs) involves } \\
\text { oxygen consumption. The interactions between } \\
\text { PMNs and microorganisms can enhance oxygen } \\
\text { consumption because of extra respiration during } \\
\text { phagocytosis by the respiratory burst resulting } \\
\text { from the electron reduction of oxygen by NADPH } \\
\text { oxidase to O-2 (Baldridge and Gerard 1933; Babior } \\
\text { et al. 1976) }\end{array}$ \\
\hline
\end{tabular}


Table 3 (continued)

\begin{tabular}{ll}
\hline Cell & Metabolic reactions in tumor \\
\hline Macrophage & Throughout tumor initiation, inflammatory macrophages by glycolytic change, \\
& HIF1a (Hypoxia Inducible Factor 1 Subunit Alpha) induction, and blocked OXPHOSP \\
& mediate NO (nitric oxide), ROI, IL-1b, and TNF expressions to promote genetic insta- \\
& bility cancer-related inflammation that contributes to tumorigenesis (Biswas 2015) \\
& The HIF1a-induced expression of the VEGFA angiogenic molecule is also seen. \\
& In TAMs of advanced tumors, activation of AMP-activated protein kinase (AMPK) \\
& through nutrient deprivation, lactate accumulation, and Th2-derived IL-4 (activates \\
& C-Myc, p53, STAT6, PGC1b), and activated PKM2 suppresses glycolysis while up- \\
& regulating OXPHOSP. This induces immunosuppressive macrophages that stimulate \\
& tumor development. Changes in the metabolism of amino acids, iron, and facts
\end{tabular}
that lead to this mechanism are often suggested (Biswas 2015)

High expression of the glycolysis pathway in tumor cells reduces amino acids and nutrients and disrupts T cells' antitumor function (Biswas 2015). Under these conditions, as Treg cells rely more on the FAO than glycolysis, they expand and modulate their immune system (Macintyre et al. 2014; Noman et al. 2014). Also, lactate produced in tumor cells similar to the kynurenine (an amino acid metabolic product) suppresses $T$ cells' antitumor activity and at the same time increases the Treg cells' (Siska and Rathmell 2015). Besides, the HIF1a produced by tumor cells increases Treg cell expansion and the induction of programmed death-1-ligand 1 (PD-L1) (Noman et al. 2014; Ben-Shoshan et al. 2008)

The decline of extracellular amino acids (through tumor absorption and other cell types), lactate aggregation, and nutritional starvation AMPK activation prevents T-cell receptor (TCR) signaling and downstream glycolysis (indicated by red T-symbol) in T-cells (e.g., CD8]+ cytotoxic T-cells). This resulted in the repression of the propagation and action of the effector. Inhibitory effect of AMPK glycolysis, stimulation of OXPHOS and FAO, and microenvironment-derived tumor stimuli (e.g., Transforming growth factor-beta (TGF- $\beta$ ), Interleukin 10 (IL-10), Kyn, and hypoxia) instead facilitate the growth of regulatory $T$ cells (Tregs). This encourages immune evasion and tumor development (Biswas 2015)

\section{Metabolic reactions in biofilm}

Ammons et al. (2017), via metabolomics by 1D 1H Nuclear Magnetic Resonance (NMR) along with immunologic evaluations, showed substantial overlap between the metabolic profile driving macrophage polarization either into the M1 phenotype or the M2 phenotype and P. aeruginosa biofilm included crucial metabolic pathways that modulate immunomodulation in macrophages like shifts between the PPP and TCA cycle and amino acid metabolism. They found that exposure of non-polarized macrophages to small metabolites from planktonic- and biofilm mode of $P$. aeruginosa resulted in distinct metabolic pathways involved in macrophage immunomodulation include increased glycolysis, uncoupling of the TCA cycle, and selective uptake and metabolism of amino acids Prendergast et al. (2017). Taken together, they found that $P$. aeruginosa biofilms relevant to specific pathological influences toward macrophages via coordinated metabolic interplays, which cause immune polarization and can involve deviation from the usual healing process of wound and expansion of a chronic host wound Prendergast et al. (2017)

The inflammatory status of leukocytes is associated with their metabolic situation. In this regard, in a study by Yamada et al. (Yamada et al. 2020), monocytes associated with biofilm show a metabolic favoring to OxPhos and less aerobic glycolysis for prompting their anti-inflammatory role that causes persistence of $S$. aureus biofilm Fuchs et al. (2020) found some metabolic pathways that are differentially increased in planktonic- and biofilm-exposed macrophages, such as purine biosynthesis glycolysis, branchedchain amino acid catabolism, inositol phosphate metabolism, as well as glycerol metabolism (Fuchs et al. 2020). Besides, these metabolic patterns show that biofilm-exposed macrophages have a hyper-inflammatory metabolic profile, diminished glycerol metabolism, and increased catabolism of amino acids and lactate compared to the planktonic exposed macrophages (Fuchs et al. 2020)

Not determined 
Table 3 (continued)

\begin{tabular}{|c|c|c|}
\hline Cell & Metabolic reactions in tumor & Metabolic reactions in biofilm \\
\hline Dendritic cell & $\begin{array}{l}\text { Tumor-associated dendritic cells (TADCs) encounter tumor-derived Damage-asso- } \\
\text { ciated molecular patterns (DAMPS) and hypoxia to upregulate glycolysis through } \\
\text { initial TBK1-IKKE and/or later PI3K-AKT-HIF1a pathway. HIF1a impairs DC maturation } \\
\text { and raises A2b and NO production (which inhibits OXPHOSP). Adenosine-A2b inter- } \\
\text { action causes immunosuppressive and pro-tumor cytokines. Rising tumor gradually } \\
\text { results in nutrient deficiency that triggers AMPK in TADCs. This, along with lactate } \\
\text { aggregation, contributes to potential glycolysis inhibition and improved control } \\
\text { of OXPHOSP in TADCs. The absorption of amino acids from the tumor microen- } \\
\text { vironment, metabolism, and MSR1-mediated lipid aggregation further facilitates } \\
\text { immunosuppressive activities to help tumor development (Biswas 2015) }\end{array}$ & Not determined \\
\hline MDSCs & $\begin{array}{l}\text { Although the critical role of nitrogen metabolism in the control of immunomodula- } \\
\text { tory activities of MDSCs in tumor situations is well-founded, very little is understood } \\
\text { about the other metabolic processes in such cells. Enhanced carbon metabolism } \\
\text { (glycolysis, glutaminolysis, and TCA activity) and its crosstalk with arginine metabo- } \\
\text { lism were observed during MDSC maturation. However, its association with both } \\
\text { AMPK and Sirtuin (SIRT) enhanced expression, which is considered to interact with } \\
\text { glycolysis, requires clarity. Increased fatty acid absorption and the FAO have also } \\
\text { recently been shown to control tumor infiltration MDSC's (Hammami et al. 2012; Liu } \\
\text { et al. 2014; Hossain et al. 2015) }\end{array}$ & Not determined \\
\hline
\end{tabular}

where it attaches to FOXP3 regulatory regions, such as its promoter and its Conserved non-coding sequence 2 (CNS2) (Rosa et al. 2015). These findings demonstrate that glucose is essential for the growth of Treg cells, for example, in the lymphatic system. Following the invasion of cancer, glucose may support Treg preservation and performance.

\section{Neutrophils}

Neutrophils rely on the glycolysis pathway and the PPP to perform their function; the PPP produces NADPH, a cofactor of NADPH oxidase, a key enzyme associated with the anti-microbial activity of neutrophils (Kumar and Dikshit 2019). Assessments of glucose6-phosphate transporter dysfunction in neutrophils have demonstrated that glycolysis has a role in controlling important neutrophil functions, such as oxidative bursting and chemotaxis (Kumar and Dikshit 2019). An essential feature of neutrophils is the development of NETs, a combination of DNA, histones, and antimicrobial peptides that traps and destroy microbes (Kumar and Dikshit 2019). Glucose absorption, glycolysis, and metabolic changes in the PPP are essential factors for developing NETs (Kumar and Dikshit 2019; Azevedo et al. 2015). NETs can capture circulating tumor cells and facilitate metastases (Kumar and Dikshit 2019; Azevedo et al. 2015). NETs have also been found to accumulate in tumor-bearing mice vascular systems correlated with pro-inflammatory adhesion molecules and cytokines that lead to tumor-induced organ dysfunction (Kumar and Dikshit 2019; Azevedo et al. 2015). All in all, these data include some insights about how the metabolic shift in neutrophils by controlling functions such as NETs could lead to cancer progression. Further characterization of cancerrelated neutrophil activities and its control by intrinsic cell metabolism is now needed.

\section{Macrophages}

TAMs have been shown to accumulate in the existence of hypoxia-inducible factor 1 alpha $(H I F 1 \alpha)$ in hypoxia environments (Henze and Mazzone 2016; Biswas 2015). Since specific glycolytic genes, such as GLUT1, Hexokinase 2 (HK2), 6-phosphofructo-2-kinase/fructose-2,6-bisphosphatase-3 (PFKFB3), and Phosphoglycerate kinase 1 (PGK1), are overexpressed, TAMs tend to use the glycolysis in environments undergoing hypoxic conditions, contributing to the development of inflammatory phenotypes that promote tumorigenesis (Biswas 2015). Latest metabolic screening has shown a preferred accumulation of many metabolites and overexpression of genes of glutamine synthesis (glutaminolysis) pathways (e.g., glutamate, alpha-ketoglutarate (AKG), Glutamic pyruvate transaminase (GPT2), and Glycine amidinotransferase (GATM)) in IL-4-induced M2 macrophages (Biswas 2015). In such cells, glutamine was necessary to protect the productive TCA cycle and the Uridine diphosphate $\mathrm{N}$-acetylglucosamine (UDP-GlcNAc) formation process needed for $\mathrm{N}$-glycosylation. Consequently, glutamine controlled the expression of M2 macrophages markers such as the CD206 and IRF4 N-glycosylated receptor, Kruppel Like Factor 4 (KLF4), and C-C Motif Chemokine Ligand 22 (CCL22). Corroborating these decisions, TAMs from Lewis lung carcinoma, which exhibit M2 
phenotype, also revealed elevated gene expression of glutamine pyruvate transaminase (GPT) and glutamate-ammonia ligase (GLUL) (Biswas 2015). In the environment of cancer, macrophages adjust their lipid profile. The Lewis lung carcinoma research revealed distinct lipid profiles in macrophages and cancerous cells (Chen et al. 2021). Leukotrienes (Leukotriene B4 (LTB4), Leukotriene C4 (LTC4), and Leukotriene D4 (LTD4)) were derived primarily from myelomonocytic cells, while prostaglandins (Prostaglandin E2 (PGE2), Prostaglandin D2 (PGD2), Prostaglandin F2 (PGF2)) were produced from both myelomonocytic and cancerous cells. In comparison, alveolar macrophages and infiltrating TAMs varied in eicosanoid profiles, the previous expressing Cyclooxygenase-1 (COX1) and 5-lipoxygenase (5-LOX) leukotrienes, whereas the latter expressed COX2 and prostaglandins.

\section{B cells}

Compared with $\mathrm{T}$ lymphocytes, B lymphocytes are highly metabolically active (Singer et al. 2018, Goodarzi et al. 2020). Each phase has a unique reliance on glucose metabolism during $B$ cells' growth, and pre-B cells are much less glucose-dependent than immature B lymphocytes (Kojima et al. 2010). After stimulation, naive B lymphocytes propagate and enhance glucose absorption and lactate output, close to what happens with T-cell stimulation (Garcia-Manteiga et al. 2011). In line with this, substantial B-cell metabolic reprogramming is needed for development of antibodies (Caro-Maldonado et al. 2014). Recently, Jellusova et al. (Jellusova et al. 2017) identified the need for high glycolytic action of germinal center B cells to sustain their development and spread in the hypoxic microenvironment. In addition to glycolysis, an improvement in mitochondrial content has been reported in germ-center B cells (Jellusova et al. 2017). In conjunction with hypoxia in the cancer microenvironment, glucose deficiency can likely benefit those B-cell phenotypes, producing an immunosuppressive environment.

\section{Biofilm}

The host immune reactions are very dynamic in function and growth because they react to immune problems and adopt the distinct metabolic settings that allow them to balance their need for energy, longevity, and molecular biosynthesis (Loftus and Finlay 2016). During immune reactions, immune cells could pass through several tissues containing various nutrients and oxygen. These cells modify their functional activities dramatically in response to activation; for example, lymphocyte cells transform to produce an inactive mode into a high proliferation and growth cell. In some situations, they form a high rate of cytokines (Loftus and Finlay 2016). These functional and micro-environmental changes in immune cells indicate significant metabolic stress but are dynamically managed by reprogramming the metabolism. Most tissues are vascularized and, as a result, filled with oxygen micro-nutrients (Loftus and Finlay 2016). In immune reactions, the local environment can change because of competition for oxygen and micronutrients. As well-seen in tumor cells, they have a tremendous appetite for micronutrients and glucose; hence, they reduce the rate of glycolysis in infiltrating lymphocyte cells in the tumor (Loftus and Finlay 2016). On the other hand, microbial infections can compete with the host immune cells for oxygen, glucose, and micronutrients (Loftus and Finlay 2016). S. aureus infections can lead to localized hypoxia because of increased oxygen consumption by bacterial and immune cells (Vitko et al. 2015). Additionally, viruses can rearrange infected host cells to regulate glucose uptake and metabolism to facilitate virus replication; that way, the level of glucose can decrease in immune cells during viral infections (Thai et al. 2014; Ripoli et al. 2010). In addition, different cells in inflammation sites can liberate enzymes including ARG and indolamine-2, 3-dioxygenase that consume micronutrients in the peripheral niches (Hirayama et al. 2009).

One of the most usual conditions of biofilm in oxic situations is a gradient of oxygen concentration, that is, at the surface layers of the biofilm, respiring bacterial cells use oxygen resulting in an oxygen limitation condition that changes the bacterial metabolism and provides anaerobic situations inside lower layers of EPS (Stewart 2003; Wu et al. 2018). Additionally, a growing body of documents shows oxygen limitation and anaerobic metabolism changes (Wu et al. 2018). For example, hypoxia situations were observed in non-healing wounds infected with obligate anaerobic microorganisms such as Clostridia and Bacteroides, which are usually isolated in chronic wounds (Dowd et al. 2008; Frank et al. 2009). In this regard, these bacteria, which require highly reduced conditions to be able to multiply, produce anoxic niches in these infected sites. Besides, direct measurement of oxygenation of skin tissue in the proximity of chronic wounds has been found and proposed to mediate the host (Ruangsetakit et al. 2010). Additionally, it has been found that neutrophils are abundant in chronic wounds (Diegelmann 2003).

The human lung is a well-aerated site, however, in some cases, such as CF, low mucociliary clearance, and thickened mucus form localized depletion of oxygen to permit overgrowth of bacteria in the human lung (Wu et al. 2018). Additionally, invading neutrophils and bacteria consume oxygen resulting in anoxic pockets within the 
infected mucosa (Downey et al. 2009; Kolpen et al. 2010; Worlitzsch et al. 2002). Studies also showed the large numbers of anaerobic microorganisms in the airways of patients with CF (Tunney et al. 2008; Guss et al. 2011; Sibley et al. 2011). These factors are under hypoxic situations within the lung mucus layer.

Oxygen concentration is a critical parameter for host healing, neutrophil function and signaling, and microbial persistence in biofilm infection (Wu et al. 2018). In a study, Wu et al. (2018) evaluated the fundamental activity of local oxygen concentration in bacterial biofilm infection etiology. Bacteria and host cells consume oxygen, modulate oxygen transport, and actively react to oxygen, causing interactions (Jesaitis et al. 2003; Campbell et al. 2014). They survived the reaction-diffusion interactions during the oxygen concentration gradients (Wu et al. 2018). The Wu et al. (2018) findings showed the co-consumption of oxygen by both host neutrophils and biofilm embedded bacteria that enhance the development of hypoxic situations in the proximity of a biofilm infection. It has been found that these diminished hypoxic levels of oxygen result in diminishing the neutrophil killing ability (Jesaitis et al. 2003; Mandell 1974) because this ability is mediated by the formation of ROS from oxygen Wu et al. (2018). Also, suppose this hypoxic situation continues, and neutrophils will be impaired in their ability to produce ROS like hypochlorous acid and hydrogen peroxide, which causes impairment of the neutrophil's ability to eliminate and control biofilm infection Wu et al. (2018).

\section{PMNs}

The $P$. aeruginosa's existence in host airways is along with infiltration of PMNs induced by chemoattractant molecules produced by airway epithelial cells and recruited leukocytes (Rada 2017). In this regard, Interleukin-8 (IL-8) could be produced by PMNs, epithelial cells, as well as macrophages and is one of the most potent recruiting chemokines for PMNs that is recognized by chemokine receptors such as $\mathrm{C}-\mathrm{X}-\mathrm{C}$ motif chemokine receptor 1 (CXCR1) and CXCR2 (Rada 2017; Guan et al. 2016). It has been found that IL-8 levels in the airways of CF patients are increased and mediated to lung damage (Colombo et al. 2005; Kim et al. 2006). Also, it has been found that increased IL-8 levels mediated to P. aeruginosa infection and detected in the exhaled breath condensate of CF patients, and it was increased in infected patients (Zoumot and Wilson 2010; Bodini et al. 2005). In CF patients, macrophages and PMNs are the primary sources of IL-8 in response to both bacterial lipopolysaccharides (LPS) and host stimuli such as IL- $1 \beta$ and TNF- $\alpha$ (Conese et al. 2003). In this regard, it remains to be further characterize whether the increased level of IL-1 $\beta$ in
CF patients is because of intrinsic NF-kB stimulation. However, CF macrophages have an intact inflammasome ability for IL- $1 \beta$ production in response to $P$. aeruginosa (Tang et al. 2012). Although PMNs produce less IL-1 $\beta$ compared to macrophages on a per cell basis, because of the higher total value of PMNs in CF airways, these cells can be a relevant source of IL-1 $\beta$ (Bakele et al. 2014). The lipid LTB4 is one of the most potent factors stimulating PMN chemotaxis and is an arachidonic acid metabolic pathway end-product and released by leukocytes as PMNs (Rada 2017). It has been found that leukotrienes exist in the sputum of CF patients and, also, PMNs from CF have diminished chemotaxis in reacting to LTB4 than PMNs from non-CF patients (Rada 2017; Lawrence and Sorrelli 1992). Also, LTB4 exists in the exhaled breath of CF patients and is increased following the infection of $P$. aeruginosa (Bodini et al. 2005).

PMNs from the airways of CF patients are different from those found in the blood of the same CF patients (Houston et al. 2013; Laval et al. 2013; Makam et al. 2009). PMNs in the CF airways of patients have increased the generation of specific metabolic and stress pathways such as CD39, CD114, C-X-C motif chemokine receptor 4 (CXCR4), as well as Receptor for advanced glycation endproducts (RAGE) (Makam et al. 2009). In this regard, it has been noted that a shift in the formation of their nutrient transporters influences the inorganic phosphate and glucose transports that are part of their adaptation to the airway of CF patients (Laval et al. 2013). PMNs from the $\mathrm{CF}$ airways have a diminished respiratory burst and changed TLRs' formation (Rada 2017). Although these cells in the site of CF airways inflammation and infection are well-documented, our knowledge about their metabolic changes is limited at this point.

Of note, about the anaerobic situation in the mucus of CF patients, Kolpin et al. (2010) hypothesized that the respiratory burst of the PMNs involves oxygen consumption. The interactions between PMNs and microorganisms can enhance oxygen consumption because of extra respiration during phagocytosis by the respiratory burst resulting from the electron reduction of oxygen by NADPH oxidase to O-2 (Baldridge and Gerard 1933; Babior et al. 1976). Kolpen et al. (2010) surveyed the respiratory burst ability to deplete oxygen. They provided a simple reaction chamber for real-time evaluation of oxygen concentration during $P$. aeruginosa phagocytosis PMNs. The respiratory burst was provided from aerobic respiration via respiratory chain blocking with potassium cyanide which causes the respiratory burst resistance to stop of the aerobic respiration in PMNs (Aj and Ml 1959). Kolpin et al. (Kolpen et al. 2010) found that CF patients' sputum includes PMNs with an active consumption of 
oxygen for the formation of O-2 which show that the respiratory burst is continues and trigger accelerated oxygen depletion due to the production of O-2 in chronically infected CF patients.

\section{Macrophage}

The Warburg effect is now understood as one of the critical features of M1 macrophages, which is essential for increasing the carbon flux in the PPP and for the formation of precursor molecules for anabolic processes and the formation of ROS (O'Neill and Pearce 2016; Torres et al. 2016). Macrophages are exposed to variety of signals, including cytokines of $\mathrm{T}$ helper and $\mathrm{B}$ lymphocyte, microbes and the host products. Interaction between macrophage and $\mathrm{T}$ and B lymphocytes has boosted host immunity and could increase the level of antimicrobial protection (Martinez et al. 2014). The role of IFN- $\gamma$ in cellular immunity against intracellular infections and the role of IL-4 in parasitic infection leads to the concept of M1 and M2 macrophages, and therefore to a broader range of immune system reactions (Martinez et al. 2014). Macrophages use OxPhos and FAO to exert their anti-inflammatory role, and the metabolic switch is carried out by global changes in gene expression in the cell (Yamada and Kielian 2019). M1 macrophages produce a highly active phosphor fructokinase two isoforms, ubiquitous phosphor fructokinase 2, and downregulate the TCA cycle enzymes that can facilitate the intracellular accumulation of glucose, citrate as well as succinate (Yamada and Kielian 2019).

Additionally, M1 macrophages generate nitric oxide via the up-regulation of iNOS, which directly inhibits OxPhos (Yamada and Kielian 2019). M2 macrophages express the less active fructokinase two phosphorus isoform, phosphate fructokinase $\mathrm{B} 1$ and upregulate CD36 to facilitate the uptake of triglycerides for fuel in the TCA cycle (Yamada and Kielian 2019; Feingold et al. 2012). Macrophages in solid tumors undergo metabolic changes via changes in the availability of nutrients, oxygen, and metabolites that coincide with the shifts in inflammatory state (Overmeire et al. 2016). Similarly, the biofilm of S. aureus produces gradients of the proton, nutrients, and oxygen that can impact macrophages' metabolism and subsequently their inflammatory state (Lone et al. 2015; Savijoki et al. 2020).

During their interaction with $P$. aeruginosa, macrophages and neutrophils generate ROS by NADPH oxidase to kill this bacterium (Genestet et al. 2014). Besides, it has been shown that immune reactions are modulated by the indoleamine 2,3-dioxygenase (IDO) of immune cells forming kynurenine via tryptophan (Genestet et al. 2014). Additionally, IDO triggers immunological tolerance via several processes, which have crucial activities in immunological phenomena like allograft acceptance, tumor camouflage, and maternofetal tolerance (Genestet et al. 2014). Furthermore, the IDO stimulation seems to be an essential mechanism for the Human immunodeficiency virus (HIV) to escape from the human immune system (Boasso et al. 2007). More importantly, the kynurenine pathway plays a role in the immune reactions during $C F$ because it has demonstrated that deficiency of IDO activity in CF, forming an imbalance of Th17/Treg that can be recovered by administration of kynurenine metabolite (Iannitti et al. 2013). Taken together, these findings show that IDO has a critical activity in homeostasis of immune reactions to $P$. aeruginosa during CF.

Currently, it has been found that $P$. aeruginosa strains utilize the kynurenine pathway for catabolizing tryptophan (Genestet et al. 2014). Additionally, some host cells generate kynurenine, which is well- defined for modulating of the immune system homeostasis (Genestet et al. 2014). In a study, Genestet et al. (2014) found that $P$. aeruginosa strains isolated from CF patients generate a high kynurenine rate. Furthermore, in $P$. aeruginosa, kynA gene, a transcriptional stimulation (involved in the kynurenine pathway) was found following contact of $P$. aeruginosa with immune cells such as neutrophils (Genestet et al. 2014). Besides, Genestet et al. (Genestet et al. 2014) via neutrophils and $P$. aeruginosa producing no $(\Delta \mathrm{kynA})$ and high rate of kynurenine $(\Delta \mathrm{kynU}) \mathrm{co}^{\mathrm{c}}$ cultures showed that kynurenine enhances the survival of this bacterium.

Interestingly, an increase in the rate of kynurenine hampers the production of ROS by stimulated neutrophils. Genestet et al. (2014), via a ROS-forming system, found that kynurenine scavenges hydrogen peroxide, as well as superoxide that occurs usually following bacterial induction, particularly in the phagosome. In summary, the kynurenine pathway enables $P$. aeruginosa to circumvent the immune reaction by scavenging ROS formation by neutrophils. Overall, these results prove the influence of kynurenine produced by bacteria on neutrophils' killing activity via ROS scavenging.

It has been found that macrophages related to the biofilm of S. aureus are polarized to an M2 phenotype. The adoptive transfer of the M1 phenotype results in attenuated biofilm burden, proposing the critical activity of macrophage inflammatory responses during biofilm infection of S. aureus (Yamada et al. 2020). The inflammatory status of leukocytes is associated with their metabolic situation. In this regard, in a study by Yamada et al. (2020), monocytes associated with biofilm show a metabolic favoring to OxPhos and less aerobic glycolysis for prompting their anti-inflammatory role that causes persistence of $S$. aureus biofilm. To change monocyte and 
reprogram immune cells' metabolism to a pro-inflammatory situation, they used a nanoparticle approach to deliver the oligomycin, an inhibitor of OxPhos, to monocyte cells (Yamada et al. 2020). Yamada et al. (2020) targeted the nanoparticle uptake to monocytes by conjugating with tuftsin and incorporated a fluorochrome to monitor nanoparticles' distribution, resulting in preferentially internalized monocytes rather than MDSCs and PMNs (Yamada et al. 2020). They delivered an oligomycin nanoparticle an in vivo $S$. aureus prosthetic joint infection model to monocytes, which notably diminished the biofilm burden by changing the monocyte metabolism and enhancing the pro-inflammatory state of infiltrating monocytes (Yamada et al. 2020). Yamada et al. (2020) showed that oligomycin injection alone had no influence on the metabolism of monocyte cells and biofilm burden and interestingly found that specific intracellular transfer of oligomycin is crucial for reprogramming the metabolic activity of monocyte cells (Yamada et al. 2020). Of note, metabolic reprogramming of monocyte cells with oligomycin nanoparticles combined with antibiotics causes the elimination of established $S$. aureus biofilm (Yamada et al. 2020). These results show that the metabolic reprogramming monocytes associated with biofilm can propose a novel therapeutic method for prosthetic joint infection.

Additionally, in a study, Hanke et al. (2013) administered the macrophage-activating peptide EP67 (Tyr-SerPhe-Lys-Asp-Met-Pro (N-methyl Leu)-d-Ala-Arg) to enhance the clearance of bacterial biofilm by performing a proinflammatory status. EP67 is an agonist for the selective reaction of the $\mathrm{C} 5 \mathrm{a}$ receptor $(\mathrm{C} 5 \mathrm{aR} / \mathrm{CD} 88)$ and preferentially triggers proinflammatory mediators from CD88+ macrophages (Hanke et al. 2013). Hanke et al. (2013) found that targeting macrophages' properties with EP67 suppresses the biofilm formation of S. aureus, proposing a novel therapeutic approach for this kind of infection. The therapeutic ability of activated macrophages shows that primary treatment with M1 macrophages significantly reduced the biofilm growth of $S$. aureus (preventive effect). Besides, activated macrophages effectively diminished the $S$. aureus burdens in established biofilm (degradative effect) (Hanke et al. 2013). The cytokine and chemokine condition produced after M1 macrophages reflects products derived from $\mathrm{T}$ lymphocytes like IL-17 and IFN- $\gamma$ and macrophages like IL-1, C-X-C motif chemokine ligand 9 (CXCL9), and $\mathrm{C}-\mathrm{C}$ motif chemokine ligand 5 (CCL5), showing the coordinate role of these immune cells (Hanke et al. 2013). This situation was only crucial in the primary glance with M1 macrophages, and the other functional influence of these factors on biofilm burdens must be evaluated (Hanke et al. 2013).
The administration of EP67 can overcome the immune dysfunction during biofilm infection, as EP67 offers the correct stimulation signals to CD88+macrophages to produce a powerful bactericidal reaction during biofilm infection (Hanke et al. 2013). In summary, these results show that immune cell-based therapy via activated macrophages can open the door to overcome the current problems mediated by biofilm infections.

Most importantly, currently, it has been found that metabolites like succinate and itaconate could be utilized as signaling molecules, metabolic intermediates, and triggers for a functional phenotypic change of macrophages (Tannahill et al. 2013; Lampropoulou et al. 2016, 2021). Previous works have found that M1 and M2 macrophages show distinct metabolite profiles such as fatty acid utilization, OxPhos, and glycolysis (Fuchs et al. 2020). Nevertheless, many evaluations are demanded to characterise macrophages' immunometabolic properties. The potential metabolic and functional differences mediated to macrophages react to invasive bacterial pathogens, particularly concerning differential phenotypic reactions of macrophages to planktonic versus biofilm growth mode (Fuchs et al. 2020). In a study, Fuchs et al. (2020) aimed to evaluate the metabolic influences of planktonic and biofilm $P$. aeruginosa on primary monocyte-derived resting (M0) macrophages via in vitro macrophage differentiation and exposure schemes (CD14+ magnetic-activated cell sorting), 1D 1H NMR metabolomics, as well as metabolic profiling analysis. In their study, M0 macrophages were differentiated from primary monocytes via macrophage colony-stimulating factor (M-CSF) before activation with planktonic and biofilm $P$. aeruginosa (Fuchs et al. 2020). After exposure, both macrophage intra- and extracellular metabolites were isolated, and 1D 1H NMR spectra were obtained, as well as metabolite profiling of these NMR spectra was calculated (Fuchs et al. 2020). Findings of Fuchs et al. (2020) proposed significant changes in metabolite profiles and show some metabolic pathways that are differentially increased in planktonicand biofilm-exposed macrophages such as purine biosynthesis glycolysis, branched-chain amino acid catabolism, inositol phosphate metabolism, as well as glycerol metabolism (Fuchs et al. 2020). Besides, these metabolic patterns showed that biofilm-exposed macrophages have a hyper-inflammatory metabolic profile, diminished glycerol metabolism, and increased catabolism of amino acids and lactate compared to the planktonic exposed macrophages (Fig. 7) (Fuchs et al. 2020). Collectedly, this study proposes novel results on the metabolic modulation of macrophages following exposure to bacteria and biofilm and opens the road to additional knowledge in the field of immunometabolism. 


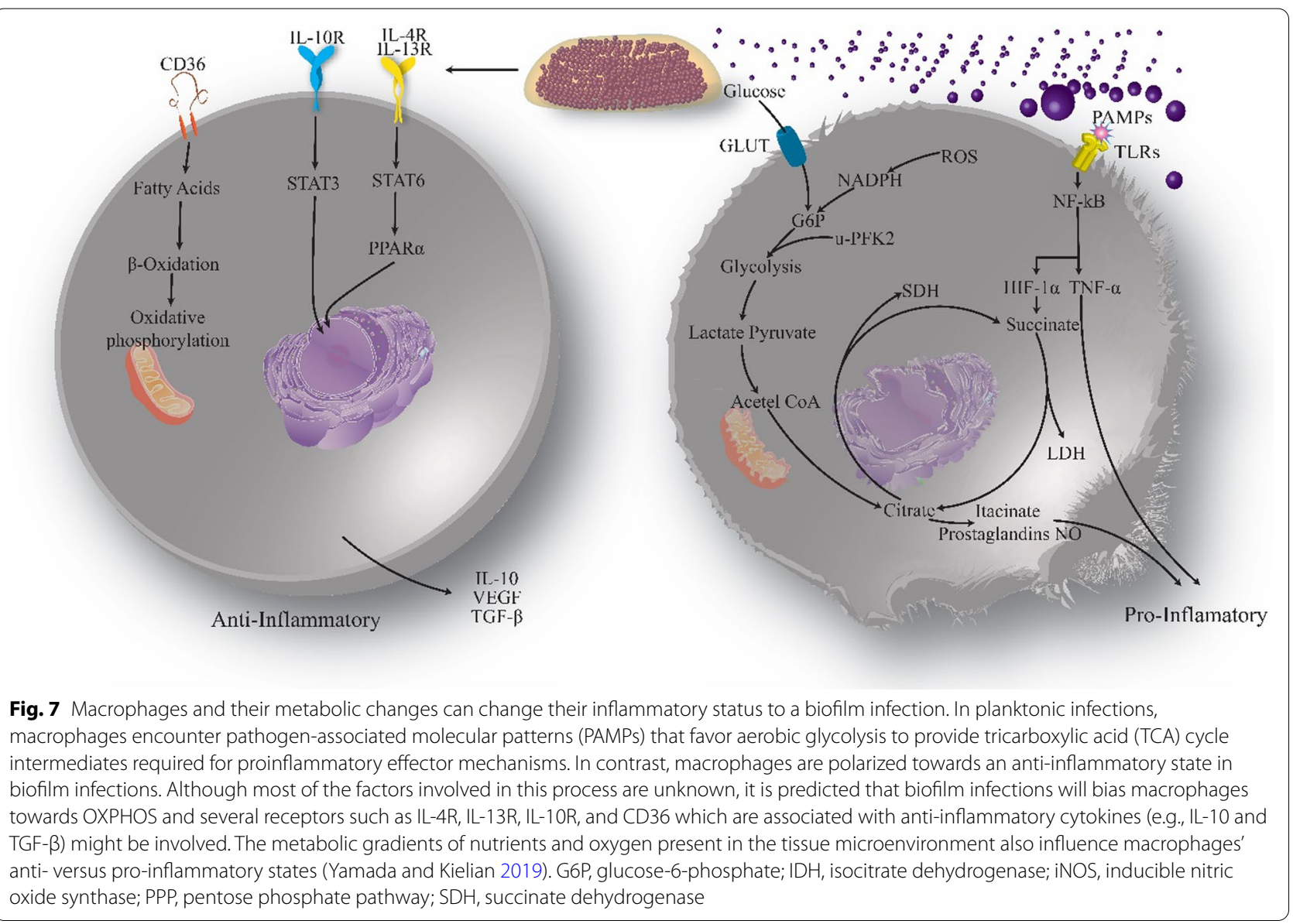

OxPhos and glycolysis are entirely coupled and act as the molecular exchange system; for example, glycolysis occurs in the host cytoplasm and forms pyruvate, which is the fuel for OxPhos. Nevertheless, as glycolysis increases, concomitant increases in the PPP are generally observed with ribose phosphate and NADPH that are needed for cellular functions, biosynthesis, and cell division (O'Neill and Pearce 2016; Loftus and Finlay 2016). This critical aspect of the immune system regulates the cell life span, influencing the population of memory lymphocytes and differentiated leukocytes against bacterial infections. Some research suggests that oxidative metabolism of phosphorylation promotes the survival of immune cells. For example, M2 macrophage (use OxPhos) has a longer lifespan, whereas M1 macrophage, which uses glycolysis, is short-lived (Loftus and Finlay 2016). These phenomena are most commonly observed in memory $\mathrm{T}$ lymphocytes, where $\mathrm{T}$ lymphocytes do not oxidize glucose at rest but instead use FAO (Wang and Green 2012).

Regarding biofilms, it was shown that macrophages that reached biofilm infection of $S$. aureus show M2 profiles (Heim et al. 2014; Heim et al. 2015). Cytokine levels decreased during biofilm device infection of mice deficient on MyD88. It was thought that other cytokines triggered during glycolysis could help maintain biofilm viability by lytic toxins of biofilm bacteria and apoptosis of effector cells. Although cytokine-induced apoptosis of macrophages and neutrophils occurs during planktonic infections, biofilm infections' static and chronic nature enhances cytokine accumulation, as seen with $S$. aureus biofilm infection (Heim et al. 2015; Heim et al. 2018).

\section{Immunometabolite itaconate}

One of the most essential components of the immune response to infection is the production of host metabolites, which control inflammation and the survival of pathogens (Tomlinson and Riquelme 2021). The researchers discovered that $S$. aureus stimulates the formation of the immunomodulatory metabolite itaconate in airway immune cells via increasing mitochondrial oxidative stress in a study were performed by Tomlinson et al. (2021). Itaconate decreased S. aureus glycolysis and proliferation to address these effects while increasing 
carbon influx via bacterial metabolic pathways that contribute to biofilm development (Tomlinson et al. 2021). Researchers found that itaconate-induced metabolic alterations were repeated in a longitudinal number of clinical isolates from a case with persistent Staphylococcal lung diseases, indicating that host immunometabolism plays a role in promoting bacterial survival throughout long-term Staphylococcal lung diseases. TCA cycle abnormalities in M1 macrophages cause deposition of TCA cycle intermediates, particularly citrate and succinate (Yamada and Kielian 2019). Succinate deposition increases HIF-1 $\alpha$ stability, epigenetic alterations, and IL-1 synthesis by suppressing $\alpha$-ketoglutarate-dependent enzymes (Loftus and Finlay 2016). Itaconate is an antimicrobial metabolite that suppresses planktonic Salmonella enterica and Mycobacterium tuberculosis development and may be generated from citrate (Loftus and Finlay 2016). Nevertheless, itaconate's antimicrobial impacts on biofilm infections have still not been investigated. Moreover, citrate is employed as a substrate for the formation of nitric oxide, reactive oxygen species, lipids, and prostaglandins. Because, macrophages are anti-inflammatory in nature during biofilm infections, they are believed to rely predominantly on OxPhos metabolism for their energy needs. Nevertheless, as a widespread survival mechanism, some bacteria, notably Yersinia Pestis and $P$. aeruginosa, disrupt itaconate by producing three enzymes: itaconyl-CoA hydratase (S) citramalyl-CoA lyase, and itaconate coenzyme A (CoA) transferase (Sasikaran et al. 2014). Moreover, P. aeruginosa uses itaconate as a carbon source, enabling it to form biofilm in the lungs of CF patients. In contrast to other opportunistic microbes, Riquelme et al. (2020) discovered that P. aeruginosa changes its metabolic and immunomodulatory features in reaction to itaconate, a common host-derived immunometabolite in the affected lung. Itaconate promotes bacterial membrane distress, which causes LPS to be downregulated and EPS to be upregulated (Riquelme et al. 2020). P. aeruginosa that has evolved to itaconate accumulates lptD mutations, which promote itaconate absorption and biofilm production. EPS could stimulate the synthesis of itaconate by myeloid cells, both systemically and in the airway system, skewing the host immune immunity to one allowed of persistent infection (Riquelme et al. 2020).

Current findings have also shown that itaconate plays a critical function in the immunometabolism of cancers (Lin et al. 2021). Itaconate has been shown to have anticancer properties in patients with colorectal cancer associated with colitis (Wang et al. 2020). IL-1 $\beta$ and CCL2 release were hindered in intestinal epithelial cells after administering dimethyl itaconate, which also diminished the penetration of M1 macrophages into the tumor microenvironment, resulting in a reduction in the elevated inflammatory processes associated with ulcerative colitis. Itaconate additionally reduced the risk of colitisassociated colorectal cancer progression by decreasing the development of cytotoxic $\mathrm{T}$ cells and subsequent infiltration of MDSCs. According to previous research, high levels of itaconate have been linked to cancer development and poor prognosis in some tumor forms (Weiss et al. 2018). The tumor milieu is dominated by tumor-associated macrophages, which may release IL-10 and growth factors that promote tumor development, aggression, and metastasis as one of the most essential elements (Vitale et al. 2019; Noy and Pollard 2014). In B16 melanoma and ID8 ovarian cancer, intraperitoneal tissue-resident macrophages were shown to upregulate Irg1 expression and raise itaconate concentrations (Weiss et al. 2018). It has been shown that itaconate increases OxPhos, resulting in ROS formation, in turn activating the mitogen-activated protein kinase (MAPK).

In contrast, when the transformation to itaconate in Irg1-/-mice was blocked, a substantial decrease in tumor volume was found in mice (Weiss et al. 2018). Itaconate also increased the production of ROS in intraperitoneal tissue-resident macrophages mediated by OxPhos, which was fueled mainly by the NADH cycle, a succinate dehydrogenase-independent process, as previously reported (Basit et al. 2018). Irg1 was produced in high concentrations in monocytes separated from the ascetic fluid of ovarian cancer patients. Similarly, other researchers noted that Irg1 performed an oncogene-like function in glioma, which resulted in an unfavorable prognosis for the patients (Pan et al. 2014). In order to thoroughly understand the immunomodulatory properties of itaconate on various kinds of cancers, further studiew are required.

Nowadays, it has been well defined that the biofilm in the wound is the main factor in the failure of therapy and expansion of non-healing, chronic human wounds. In a study, Ammons et al. (2017) surveyed how metabolite competition and condition between macrophages and biofilm produced by $P$. aeruginosa promotes M1 polarization of macrophages and skews macrophages into an M2 phenotype. Ammons et al. (2017), via metabolomics by $1 \mathrm{D} 1 \mathrm{H}$ Nuclear magnetic resonance (NMR) along with immunologic evaluations, showed substantial overlap between the metabolic profile driving macrophage polarization either into the M1 phenotype or the M2 phenotype and showed that the P. aeruginosa biofilm included crucial metabolic pathways that modulate immunomodulation in macrophages like shifts between the PPP and 
TCA cycle and amino acid metabolism Rada (2017). They found that exposure of non-polarized macrophages to small metabolites from planktonic- and biofilm mode of $P$. aeruginosa resulted in distinct metabolic pathways involved in macrophage immunomodulation including increased glycolysis, uncoupling of the TCA cycle, and selective uptake and metabolism of amino acids Rada (2017). Additionally, Ammons et al. (2017) used biofilmtargeted therapeutics to handle macrophage polarization immunomodulation during biofilm infection to enhance a shift from an M1 phenotype to an M2 phenotype in un-activated macrophages. Taken together, they found that $P$. aeruginosa biofilms relevant to specific pathological influences toward macrophages via coordinated metabolic interplays, which cause immune polarization and can involve deviation from the usual healing process of wound and expansion of a chronic host wound Rada (2017). These results can lead to new methods for treatment and prevention of non-healing wounds, such as developing treatment protocols using immune metabolites.

\section{Immunometabolism as a novel therapeutic approach}

The idea that metabolic shifts modify the phenotype and role of immune cells in the tumor microenvironment is supported and assumed to at least partially lead to acquired resistance to tumor immunotherapy (Guo et al. 2019). The critical objective of immunotherapy is to counteract the suppression of the immune system by efficiently mobilizing cancer-specific T-effector cells and producing $\mathrm{T}$-memory cells that enable long-term immune-mediated removal or regulation of tumors (Guo et al. 2019). Because metabolic programming encourages the production and functionality of immune cells, the use of metabolic medications may provide new strategies to promote tumor immunotherapy (Guo et al. 2019). Evidence is emerging that metabolic control, e.g., repression of cholesterol esterification, suppressing adenosine monophosphate transfer, and targeting imbalanced lipid aggregation in tumor-infiltration dendritic cells, can be used to improve or normalize immune functions for improved cancer care (Yang et al. 2016; Allard et al. 2013; Cubillos-Ruiz et al. 2015; Herber et al. 2010). A complete list of immunometabolism-based therapies for cancer is provided in previous studies (Beezhold and Byersdorfer 2018, Mockler et al. 2014, Singer et al. 2018; Guo et al. 2019; Mazumdar et al. 2020).

Immune cell activation and sufficient differentiation are crucial in various infections (Ng et al. 2013, 2002). Characterizing different signaling pathways and transcription patterns in immune cell subsets can lead to the identification and offer of new targets for improving the treatment of biofilm infections. It has also been revealed that the reprogramming of cellular metabolic pathways has a vital role in triggering and controlling immune cell reactions (Pearce and Pearce 2013). Accordingly, more attention has been given to interrogating and manipulating human immune cells' metabolic reactions to develop new therapeutic methods in infectious diseases. Once immune cells are activated, metabolic rescheduling and nutrient uptake changes are essential for biosynthetic pathways in terms of cell division and function (Pearce and Pearce 2013). This mode is defined by increased glutamine metabolism, glycolytic rate, PPP, and the formation of nucleic acids, proteins, and lipids (Polat 2016). For example, the metabolic programs used by different $\mathrm{T}$ cell subgroups appear to have distinct features that are essential to support the strengthening and function of their lines (Slack et al. 2015).

In the case of biofilm, $S$. aureus has been shown to stimulate the development of MDSCs strongly, and MDSCs cause severe infections (Ost et al. 2016). Nevertheless, MDSCs were mediated to an enhanced outcome like $P$. aeruginosa in CF patients (Ost et al. 2016). A powerful therapeutic approach can target MDSCs in $S$. aureus biofilm implant infections. Further knowledge remains on how to translate these results into therapeutic approaches. For example, the tyrosine-kinase inhibitors, e.g., Sunitinib, interfere with STAT3 signaling and reduce MDSCs (Ost et al. 2016). The same outcome could also be achieved using an active metabolite of vitamin (alltrans-retinoic acid (ATRA)) (Ost et al. 2016).

This review aimed to provide an overview of the emergence of immunometabolism in infectious diseases, especially biofilm infections. Therefore, we have provided examples in various studies in which immune cell metabolism is involved in immune responses to bacterial infections, especially biofilm infections. The dynamic nature of metabolic programming among immune cells is closely related to their flexibility and function. The metabolism pathways have recently been targeted for some autoimmune and inflammatory diseases (for example, calcineurin inhibitors (cyclosporine and FK506 suppress effector and regulator cells), and metabolic therapy approaches can offer a promising field of research into the treatment and control of infectious diseases (Shen et al. 2016; Harrison et al. 2007; Huang and Perl 2018, 2019). A growing body of evidence shows how metabolism inhibitors (glycolysis, glutamine metabolism, and fatty acid oxidation) can regulate human immune responses and cure immune-mediated pathogenesis. For example, drugs that target IL-1 $\beta$ exhibited promising results in clinical trials (Ayres 2020). Targeting immune and metabolic regulators can represent a promising novel approach for curing and preventing bacterial infections. Various studies have examined metabolic 
alterations during infection and highlighted the importance of metabolism in host defense to pathogens.

\section{Conclusion}

Biofilm plays a crucial role in the prognosis and progression of various bacterial infections that are often associated with chronic states and cause long-term problems for patients. Mechanisms of stability and resistance of biofilm bacteria present unique and evolving challenges to patients and physicians. For this reason, biofilm research is receiving more attention. Interestingly, biofilm infections have been shown to modify the host's peripheral environment. Most importantly, it has been shown that biofilm infections can change the inflammatory immune reactions, as evidenced by macrophage polarization and recruitment of MDSCs, to anti-inflammatory modes. Overall, these findings show that biofilm infections can change immune cells' metabolism, affecting the development and activation of immune reactions (Table 3). Innovative therapeutic agents in infections are crucial to overcoming biofilm-associated infections' severe complications. We suggest that more attention should be given to interrogating and manipulating the metabolic reactions in immune cells to develop new therapeutic methods in biofilm infections. Besides, combining bacteriotherapy approaches with chemotherapy can help defeat tumor heterogeneity accompanied by malignancy, drug resistance, and metastasis.

\footnotetext{
Abbreviations

EPS: Extracellular polymeric substances; MDSCs: Myeloid-derived suppressor cells; LPS: Lipopolysaccharides; OxPhos: Oxidative phosphorylation; IL-17: Interleukin-17; ETBF: Enterotoxigenic Bacteroides fragilis; IBD: Inflammatory bowel diseases; IL-6: Interleukin 6; STAT3: Signal transducer and activator of transcription 3; OMV: Outer membrane vesicles; PCR: polymerase chain reaction; IgG: Immunoglobulin G; OMP: Outer membrane protein; Cag: Cytotoxinassociated gene; PAl: Pathogenicity island; TCA: Ricarboxylic acid; NADPH: Nicotinamide adenine dinucleotide phosphate; ATP: Adenosine Triphosphate; FAD: Flavin adenine dinucleotide; LAT1: L-Type Amino Acid Transporter; APCs: Antigen-presenting cells; CTL: Cytotoxic T lymphocytes; IL-2: Interleukin-2;

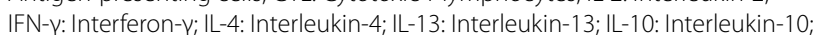
IL-23: Interleukin-23; TNF-a: Tumor necrosis factor-a; IgG2a: Immunoglobulin G2a; TCR: T-cell receptor; IL-12: Interleukin 12; IL-18: Interleukin-18; IL-5: Interleukin-5; IgE: Immunoglobulin E; TGF- $\beta$ : Transformation of the growth factor- $\beta$; IgM: Immunoglobulin M; IgA: Immunoglobulin A; Tregs: Regulatory T cells; FOXP3: Forkhead box protein P3; CTLA4: Cytotoxic T-lymphocyte antigen 4; M1: Pro-inflammatory macrophages; M2: Anti-inflammatory macrophages; RBPJ: Recombination signal binding protein for the immunoglobulin kappa $J$ region; ARG1: Arginase 1; IRF4: Interferon regulatory factor 4; SOCS3: Cytokine signaling suppressor 3; CCL1: Chemokine ligand 1; IL-1: Interleukin-1; PMNs: Polymorphonuclear leukocytes; ROS: Reactive oxygen species; NET: Neutrophil extracellular trap; QS: Quorum sensing; AHL: N-acyl homoserine lactone; CXCL2: C-X-C Motif Chemokine Ligand 2; INOS: Inducible nitric oxide synthase; TLRs: Toll-like receptors; GM-CSF: Granulocyte-macrophage colony-stimulating factor; VEGF: Vascular endothelial growth factor; G-CSF: Granulocyte-colony stimulating factor; FnBP: Fibronectin-binding protein; Cas8: Caspase 8; SCV: Small colony variant; IL-1R: IL-1 receptors; MyD88: Myeloid differentiation primary response 88 ; CXCL1: Chemokine (C-X-C motif) ligand 1; GAPDH: Glyceraldehyde-3-phosphate dehydrogenase; NFAT:
}

Nuclear factor of activated T cells; PI3K: Phosphatidylinositol 3-kinase; AKT: Protein kinase B; MTORC1: Mammalian target of rapamycin complex 1; CNS2: Conserved non-coding sequence 2; PPP: Pentose phosphate pathway; TAMs: Tumor-associated macrophages; HIF1 a: Hypoxia-inducible factor 1 alpha; CF: Cystic fibrosis; IL-8: Interleukin 8; CXCR1: C-X-C motif chemokine receptor 1; CXCR2: C-X-C motif chemokine receptor 2; LTB4: Lipid leukotriene B4; CXCR4: C-X-C motif chemokine receptor 4; RAGE: Receptor for Advanced Glycation Endproducts; HIV: Human Immunodeficiency Virus; NMR: Nuclear Magnetic Resonance; M-CSF: Macrophage colony-stimulating factor.

\section{Acknowledgements}

The authors thank Shawn Lewenza (Athabasca University) for the careful proofreading of the manuscript. The authors also thank all persons who helped revise and complete this manuscript.

\section{Authors' contributions}

$\mathrm{RM}, \mathrm{HM}$, and NS participated in the study design, wrote the draft, and collected the documentation materials. YAY and SK participated in the study design and helped revise the draft. All authors read and approved the manuscript.

\section{Funding \\ None.}

Availability of data and materials

Not applicable.

\section{Declarations}

Ethics approval and consent to participate

Not applicable.

\section{Consent for publication \\ Not applicable.}

\section{Competing interests}

The authors declare that they have no known competing financial interests or personal relationships that could have influenced the work reported in this paper.

\section{Author details}

${ }^{1}$ Department of Microbiology, School of Medicine, Hamadan University of Medical Sciences, Hamadan, Iran. ${ }^{2}$ Venom and Biotherapeutics Molecules Lab, Medical Biotechnology Department, Biotechnology Research Center, Pasteur Institute of Iran, Tehran, Iran. ${ }^{3}$ Department of Microbiology, School of Medicine, Shahid Sadoughi University of Medical Sciences, Yazd, Iran. ${ }^{4}$ Department of Medical Biotechnology, School of Advanced Medical Sciences and Technologies, Hamadan University of Medical Sciences, Hamadan, Iran. ${ }^{5}$ Research Center for Molecular Medicine, School of Medicine, Hamadan University of Medical Sciences, Hamadan, Iran. ${ }^{6}$ Department of Microbiology, School of Medicine, Kermanshah University of Medical Sciences, Kermanshah, Iran. ${ }^{7}$ Gastrointestinal and Liver Diseases Research Center, Iran University of Medical Sciences, Tehran, Iran. ${ }^{8}$ Department of Virology, School of Medicine, Iran University of Medical Sciences, Tehran, Iran.

Received: 13 August 2021 Accepted: 10 January 2022

Published online: 29 January 2022

\section{References}

Abadi ATB. Strategies used by Helicobacter pylori to establish persistent infection. World J Gastroenterol. 2017;23:2870-82.

Abdi M, Mirzaei R. Iran without mandatory quarantine and with social distancing strategy against coronavirus disease (COVID-19). Health Security. 2020;18:257-9.

Agarwal A, et al. Flow cytometric analysis of Th1 and Th2 cytokines in PBMCs as a parameter of immunological dysfunction in patients of superficial transitional cell carcinoma of bladder. Cancer Immunol Immunother. 2006;55:734-43. 
Aj S, MI K. The biochemical basis of phagocytosis. I. Metabolic changes during the ingestion of particles by polymorphonuclear leukocytes. J Biol Chem. 1959;234:1355-62.

Akira S, Uematsu S, Takeuchi O. Pathogen recognition and innate immunity. Cell. 2006;124:783-801.

Alboslemy T, Yu B, Rogers T, Kim M-H. Staphylococcus aureus biofilmconditioned medium impairs macrophage-mediated antibiofilm immune response by upregulating KLF2 expression. Infect Immun. 2019;87:e00643-e1618.

Alhede $M$, et al. The origin of extracellular DNA in bacterial biofilm infections in vivo. Pathog Dis. 2020;78:ftaa018.

Allard B, Pommey S, Smyth MJ, Stagg J. Targeting CD73 enhances the antitumor activity of anti-PD-1 and anti-CTLA-4 mAbs. Clin Cancer Res. 2013;19:5626-35

Allen-Vercoe E, Strauss J, Chadee K. Fusobacterium nucleatum: an emerging gut pathogen? Gut Microbes. 2011;2:294-8.

Almuhaideb A, Papathanasiou N, Bomanji J. 18F-FDG PET/CT imaging in oncology. Ann Saudi Med. 2011;31:3-13.

Alves P, et al. (2018) Interaction between Staphylococcus aureus and Pseudomonas aeruginosa is beneficial for colonisation and pathogenicity in a mixed-biofilm

Ammons MCB, Fuchs AL, Tripet BP, Copie V. Metabolic immunomodulation of macrophage polarization by Pseudomonas aeruginosa biofilms. FASEB J. 2017:31:622.629-622.629.

Annunziato $F$, et al. Phenotypic and functional features of human Th17 cells. J Exp Med. 2007;204:1849-61.

Arciola CR, Campoccia D, Montanaro L (2018) Implant infections: adhesion, biofilm formation and immune evasion. Nat Rev Microbiol. 1

Ayres JS. Immunometabolism of infections. Nat Rev Immunol. 2020;20:79-80.

Azevedo EP, et al. A metabolic shift toward pentose phosphate pathway is necessary for amyloid fibril- and phorbol 12-myristate 13-acetateinduced neutrophil extracellular trap (NET) formation. J Biol Chem. 2015;290:22174-83.

Babior BM, Curnutte J, McMurrich BJ. The particulate superoxide-forming system from human neutrophils. Properties of the system and further evidence supporting its participation in the respiratory burst. J Clin Investig. 1976;58:989-96.

Bakele $\mathrm{M}$, et al. Localization and functionality of the inflammasome in neutrophils. J Biol Chem. 2014;289:5320-9.

Baldridge C, Gerard R. The extra respiration of phagocytosis. Am J Physiol. 1933;103:235-6.

Balkwill F, Charles KA, Mantovani A. Smoldering and polarized inflammation in the initiation and promotion of malignant disease. Cancer Cell. 2005;7:211-7.

Bartosik AA, et al. Transcriptional profiling of ParA and ParB mutants in actively dividing cells of an opportunistic human pathogen Pseudomonas aeruginosa. PLoS ONE. 2014;9:e87276.

Basit F, Mathan T, Sancho D, de Vries IJM. Human dendritic cell subsets undergo distinct metabolic reprogramming for immune response. Front Immunol. 2018;9:2489.

Bass H, Yamashita N, Clement L. Heterogeneous mechanisms of human cytotoxic T lymphocyte generation. II. Differential effects of IL-6 on the helper cell-independent generation of CTL from CD8+ precursor subpopulations. J Immunol. 1993;151:2895-903.

Battista MJ, et al. Feasibility of induced metabolic bioluminescence imaging in advanced ovarian cancer patients: first results of a pilot study. J Cancer Res Clin Oncol. 2016;142:1909-16.

Bayat Mokhtari R, et al. Combination therapy in combating cancer. Oncotarget. 2017:8:38022-43.

Beezhold K, Byersdorfer CA. Targeting immuno-metabolism to improve anticancer therapies. Cancer Lett. 2018;414:127-35.

Belkaid Y, Hand TW. Role of the microbiota in immunity and inflammation. Cell. 2014;157:121-41.

Benoit DS, Koo H (2016) Targeted, triggered drug delivery to tumor and biofilm microenvironments. In. Future Medicine

Ben-Shoshan J, Maysel-Auslender S, Mor A, Keren G, George J. Hypoxia controls CD4+ CD25+ regulatory T-cell homeostasis via hypoxia-inducible factor-1a. Eur J Immunol. 2008;38:2412-8.

Bettelli $\mathrm{E}$, et al. Reciprocal developmental pathways for the generation of pathogenic effector TH 17 and regulatory T cells. Nature. 2006;441:235-8.
Birkenhauer E, Neethirajan S, Weese JS. Collagen and hyaluronan at wound sites influence early polymicrobial biofilm adhesive events. BMC Microbiol. 2014;14:1-11.

Biswas SK. Metabolic reprogramming of immune cells in cancer progression. Immunity. 2015:43:435-49.

Blagosklonny MV. Overcoming limitations of natural anticancer drugs by combining with artificial agents. Trends Pharmacol Sci. 2005;26:77-81.

Blanchette KA, Orihuela CJ. Future perspective on host-pathogen interactions during bacterial biofilm formation within the nasopharynx. Future Microbiol. 2012;7:227-39.

Boasso A, et al. HIV inhibits CD4+ T-cell proliferation by inducing indoleamine 2, 3-dioxygenase in plasmacytoid dendritic cells. Blood. 2007;109:3351-9.

Bodini A, et al. Biomarkers of neutrophilic inflammation in exhaled air of cystic fibrosis children with bacterial airway infections. Pediatr Pulmonol. 2005:40:494-9.

Brassart-Pasco S, et al. Tumor microenvironment: extracellular matrix alterations influence tumor progression. Front Oncol. 2020. https://doi.org/ 10.3389/fonc.2020.00397.

Bronte V, Serafini P, Apolloni E, Zanovello P. Tumor-induced immune dysfunctions caused by myeloid suppressor cells. J Immunother. 2001;24:431-46.

Buck MD, O'sullivan D, Pearce EL. T cell metabolism drives immunity. J Exp Med. 2015:212:1345-60.

Bui LM, Conlon BP, Kidd SP. Antibiotic tolerance and the alternative lifestyles of Staphylococcus aureus. Essays Biochem. 2017;61:71-9.

Bunt SK, Clements VK, Hanson EM, Sinha P, Ostrand-Rosenberg S. Inflammation enhances myeloid-derived suppressor cell cross-talk by signaling through Toll-like receptor 4. J Leukoc Biol. 2009;85:996-1004.

Campbell EL, et al. Transmigrating neutrophils shape the mucosal microenvironment through localized oxygen depletion to influence resolution of inflammation. Immunity. 2014;40:66-77.

Campoccia D, Mirzaei R, Montanaro L, Arciola CR. Hijacking of immune defences by biofilms: a multifront strategy. Biofouling. 2019;35:1055-74

Camus M, Galon J (2010) Memory T-cell responses and survival in human cancer: remember to stay alive. In: Memory T Cells. Springer, pp. 166-177

Cantor JR, Sabatini DM. Cancer cell metabolism: one hallmark, many faces. Cancer Discov. 2012;2:881-98.

Caro-Maldonado A, et al. Metabolic reprogramming is required for antibody production that is suppressed in anergic but exaggerated in chronically BAFF-exposed B cells. J Immunol. 2014;192:3626-36.

Carracedo A, Cantley LC, Pandolfi PP. Cancer metabolism: fatty acid oxidation in the limelight. Nat Rev Cancer. 2013;13:227-32.

Carron MA, Tran VR, Sugawa C, Coticchia JM. Identification of Helicobacter pylori biofilms in human gastric mucosa. J Gastrointestinal Surg. 2006;10:712-7.

Cavenee WK, White RL. The genetic basis of cancer. Sci Am. 1995;272:72-9.

Cellini L. Helicobacter pylori: a chameleon-like approach to life. World J Gastroenterol. 2014:20:5575-82.

Cellini $\mathrm{L}$, et al. Dynamic colonization of Helicobacter pylori in human gastric mucosa. Scand J Gastroenterol. 2008:43:178-85.

Chakravarthi BVSK, Nepal S, Varambally S. Genomic and epigenomic alterations in cancer. Am J Pathol. 2016:186:1724-35.

Cham CM, Driessens G, O'Keefe JP, Gajewski TF. Glucose deprivation inhibits multiple key gene expression events and effector functions in CD8+ T cells. Eur J Immunol. 2008;38:2438-50.

Chan WY, et al. Coccoid forms of Helicobacter pylori in the human stomach. Am J Clin Pathol. 1994;102:503-7.

Chang $\mathrm{C}-\mathrm{H}$, et al. Posttranscriptional control of T cell effector function by aerobic glycolysis. Cell. 2013;153:1239-51.

Chang $\mathrm{C}-\mathrm{H}$, et al. Metabolic competition in the tumor microenvironment is a driver of cancer progression. Cell. 2015;162:1229-41.

Chanmee T, Ontong P, Konno K, Itano N. Tumor-associated macrophages as major players in the tumor microenvironment. Cancers. 2014;6:1670-90.

Chen D, Zhang X, Li Z, Zhu B. Metabolic regulatory crosstalk between tumor microenvironment and tumor-associated macrophages. Theranostics. 2021;11:1016-30. 
Colamatteo A, et al. Metabolism and autoimmune responses: the microRNA connection. Front Immunol. 2019. https://doi.org/10.3389/fimmu.2019. 01969.

Cole SP, Harwood J, Lee R, She R, Guiney DG. Characterization of monospecies biofilm formation by Helicobacter pylori. J Bacteriol. 2004;186:3124-32.

Colombo MP, Piconese S. Regulatory T-cell inhibition versus depletion: the right choice in cancer immunotherapy. Nat Rev Cancer. 2007;7:880-7.

Colombo MP, Piconese S. Regulatory-T-cell inhibition versus depletion: the right choice in cancer immunotherapy. Nat Rev Cancer. 2007;7:880-7.

Colombo C, et al. Cytokine levels in sputum of cystic fibrosis patients before and after antibiotic therapy. Pediatr Pulmonol. 2005;40:15-21.

Coman DR. Adhesiveness and stickiness: two independent properties of the cell surface. Can Res. 1961;21:1436-8.

Condamine T, Gabrilovich DI. Molecular mechanisms regulating myeloidderived suppressor cell differentiation and function. Trends Immunol. 2011;32:19-25.

Condamine T, Ramachandran I, Youn J-I, Gabrilovich DI. Regulation of tumor metastasis by myeloid-derived suppressor cells. Annu Rev Med. 2015;66:97-110

Conese M, Copreni E, Di Gioia S, De Rinaldis P, Fumarulo R. Neutrophil recruitment and airway epithelial cell involvement in chronic cystic fibrosis lung disease. J Cystic Fibrosis. 2003;2:129-35.

Costerton JW, Stewart PS, Greenberg EP. Bacterial biofilms: a common cause of persistent infections. Science. 1999:284:1318-22.

Coticchia JM, et al. Presence and density of Helicobacter pylori biofilms in human gastric mucosa in patients with peptic ulcer disease. J Gastrointestinal Surg. 2006:10:883-9.

Coussens LM, Werb Z. Inflammation and cancer. Nature. 2002;420:860-7.

Cua DJ, et al. Interleukin-23 rather than interleukin-12 is the critical cytokine for autoimmune inflammation of the brain. Nature. 2003;421:744-8.

Cubillos-Ruiz JR, et al. ER stress sensor XBP1 controls anti-tumor immunity by disrupting dendritic cell homeostasis. Cell. 2015;161:1527-38.

Curiel TJ, et al. Specific recruitment of regulatory $T$ cells in ovarian carcinoma fosters immune privilege and predicts reduced survival. Nat Med. 2004;10:942-9.

Dai J, El Gazzar M, Li GY, Moorman JP, Yao ZQ. Myeloid-derived suppressor cells: paradoxical roles in infection and immunity. J Innate Immun. 2015:7:116-26.

Dandekar RC, Kingaonkar AV, Dhabekar GS. Role of macrophages in malignancy. Ann Maxillofac Surg. 2011;1:150

Darmon E, Leach DRF. Bacterial genome instability. Microbiol Mol Biol Rev. 2014;78:1-39.

De Rosa V, et al. Glycolysis controls the induction of human regulatory $T$ cells by modulating the expression of FOXP3 exon 2 splicing variants. Nat Immunol. 2015;16:1174-84.

Dejea CM, Sears CL. Do biofilms confer a pro-carcinogenic state? Gut Microbes. 2016:7:54-7.

Dejea CM, et al. Microbiota organization is a distinct feature of proximal colorectal cancers. Proc Natl Acad Sci. 2014;111:18321-6.

Dejea CM, et al (2014) Microbiota organization is a distinct feature of proximal colorectal cancers 111: 18321-18326

Del Castillo E, Meier R, Chung M. The microbiomes of pancreatic and duodenum tissue overlap and are highly subject specific but differ between pancreatic cancer and noncancer subjects. Cancer Epidemiol Prev Biomark. 2019;28:370-83.

Dey P. Cancer nucleus: morphology and beyond. Diagn Cytopathol. 2010:38:382-90.

Diaz-Montero CM, et al. Increased circulating myeloid-derived suppressor cells correlate with clinical cancer stage, metastatic tumor burden and doxorubicin-cyclophosphamide chemotherapy. Cancer Immunol Immunother. 2009;58:49-59.

Diegelmann RF. Excessive neutrophils characterize chronic pressure ulcers. Wound Repair Regen. 2003;11:490-5.

Dinarello CA. IL-18: a TH1-inducing, proinflammatory cytokine and new member of the IL-1 family. J Allergy Clin Immunol. 1999;103:11-24.

Dominiak A, Chełstowska B, Olejarz W, Nowicka G. Communication in the cancer microenvironment as a target for therapeutic interventions. Cancers (basel). 2020;12:1232.

Donaldson K, Murphy FA, Duffin R, Poland CA. Asbestos, carbon nanotubes and the pleural mesothelium: a review of the hypothesis regarding the role of long fibre retention in the parietal pleura, inflammation and mesothelioma. Part Fibre Toxicol. 2010;7:5.

Donelli G, Vuotto C, Cardines R, Mastrantonio P. Biofilm-growing intestinal anaerobic bacteria. FEMS Immunol Med Microbiol. 2012;65:318-25.

Donlan RM. Biofilms: microbial life on surfaces. Emerg Infect Dis. 2002;8:881-90.

Dowd SE, et al. Survey of bacterial diversity in chronic wounds using pyrosequencing, DGGE, and full ribosome shotgun sequencing. BMC Microbiol. 2008:8:43.

Downey D, Bell S, Elborn J. Neutrophils in cystic fibrosis. Thorax. 2009;64:81-8.

Drewes $\mathrm{J}$, et al. High-resolution bacterial $16 \mathrm{~S}$ rRNA gene profile meta-analysis and biofilm status reveal common colorectal cancer consortia. NPJ Biofilms Microbiomes. 2017;3:1-12.

Drewes JL, et al. High-resolution bacterial 16S rRNA gene profile meta-analysis and biofilm status reveal common colorectal cancer consortia. NPJ Biofilm Microbiome. 2017;3:34

Dunn GP, Bruce AT, Ikeda H, Old L, Schreiber RD. Cancer immunoediting: from immunosurveillance to tumor escape. Nat Immunol. 2002;3:991-8.

Dunn GP, Old LJ, Schreiber RD. The immunobiology of cancer immunosurveillance and immunoediting. Immunity. 2004;21:137-48.

Dunn GP, et al. A critical function for type I interferons in cancer immunoediting. Nat Immunol. 2005;6:722-9.

Edwards AM, Grossman TJ, Rudney JD. Fusobacterium nucleatum transports noninvasive Streptococcus cristatus into human epithelial cells. Infect Immun. 2006;74:654-62.

Eisenreich W, Heesemann J, Rudel T, Goebel W. Metabolic host responses to infection by intracellular bacterial pathogens. Front Cell Infect Microbiol. 2013;3:24-24.

Eisenreich W, Rudel T, Heesemann J, Goebel W. How viral and intracellular bacterial pathogens reprogram the metabolism of host cells to allow their intracellular replication. Front Cell Infect Microbiol. 2019. https:// doi.org/10.3389/fcimb.2019.00042.

El-Awady A, et al. Polymicrobial synergy within oral biofilm promotes invasion of dendritic cells and survival of consortia members. NPJ Biofilms Microbiomes. 2019:5:1-12.

Erard F, Wild M-T, Garcia-Sanz JA, Le Gros G. Switch of CD8 T cells to noncytoIytic CD8-CD4-cells that make TH2 cytokines and help B cells. Science. 1993:260:1802-5.

Escoll P, Buchrieser C. Metabolic reprogramming of host cells upon bacterial infection: why shift to a Warburg-like metabolism? FEBS J. 2018;285:2146-60.

Escoll P, Buchrieser C. Metabolic reprogramming: an innate cellular defence mechanism against intracellular bacteria? Curr Opin Immunol. 2019;60:117-23.

Eugenin EA. Role of cell-to-cell communication in cancer: new features, insights, and directions. Wiley Online Library: Hoboken; 2019.

Fehérvari Z, Sakaguchi S. CD4+ Tregs and immune control. J Clin Investig. 2004;114:1209-17.

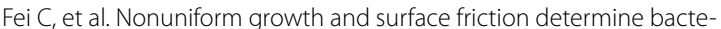
rial biofilm morphology on soft substrates. Proc Natl Acad Sci. 2020;117:7622-32.

Feingold KR, et al. Mechanisms of triglyceride accumulation in activated macrophages. J Leukoc Biol. 2012;92:829-39.

Finkel T. The metabolic regulation of aging. Nat Med. 2015;21:1416.

Flemer $\mathrm{B}$, et al. The oral microbiota in colorectal cancer is distinctive and predictive. Gut. 2018;67:1454-63.

Flemming $\mathrm{H}-\mathrm{C}$, et al. Biofilms: an emergent form of bacterial life. Nat Rev Microbiol. 2016;14:563.

Flemming $\mathrm{H}-\mathrm{C}$, et al. Who put the film in biofilm? The migration of a term from wastewater engineering to medicine and beyond. Npj Biofilms Microbiomes. 2021;7:1-5.

Foreman A, et al. Adaptive immune responses in Staphylococcus aureus biofilm-associated chronic rhinosinusitis. Allergy. 2011;66:1449-56.

Frank DN, et al. Microbial diversity in chronic open wounds. Wound Repair Regen. 2009;17:163-72.

Franz S, Rammelt S, Scharnweber D, Simon JC. Immune responses to implants-a review of the implications for the design of immunomodulatory biomaterials. Biomaterials. 2011;32:6692-709. 
Frauwirth KA, et al. The CD28 signaling pathway regulates glucose metabolism. Immunity. 2002;16:769-77.

Fridlender ZG, et al. Polarization of tumor-associated neutrophil phenotype by TGF-beta: "N1"versus"N2"TAN. Cancer Cell. 2009;16:183-94.

Fuchs AL, et al. Pseudomonas aeruginosa planktonic- and biofilm-conditioned media elicit discrete metabolic responses in human macrophages. Cells. 2020;9:2260.

Gabrilovich DI, Nagaraj S. Myeloid-derived suppressor cells as regulators of the immune system. Nat Rev Immunol. 2009:9:162-74.

Gao Y, et al. Renal cancer-derived exosomes induce tumor immune tolerance by MDSCs-mediated antigen-specific immunosuppression. Cell Commun Signaling. 2020;18:1-14.

Garcia-Manteiga JM, et al. Metabolomics of B to plasma cell differentiation. J Proteome Res. 2011;10:4165-76.

Genard G, Wera AC, Huart C, Le Calve B, Penninckx S. Proton irradiation orchestrates macrophage reprogramming through NFkB signaling. Cell Death Dis. 2018;9:728.

Genestet C, et al. Scavenging of reactive oxygen species by tryptophan metabolites helps Pseudomonas aeruginosa escape neutrophil killing. Free Radical Biol Med. 2014;73:400-10.

Gerriets VA, et al. Foxp3 and Toll-like receptor signaling balance T reg cell anabolic metabolism for suppression. Nat Immunol. 2016;17:1459-66.

González JF, Hahn MM, Gunn JS. Chronic biofilm-based infections: skewing of the immune response. Pathog Dis. 2018;76:fty023.

Goodarzi P, et al. Coronavirus disease 2019 (COVID-19): immunological approaches and emerging pharmacologic treatments. Int Immunopharmacol. 2020:88:106885.

Grassi L, Maisetta G, Esin S, Batoni G. Combination strategies to enhance the efficacy of antimicrobial peptides against bacterial biofilms. Front Microbiol. 2017. https://doi.org/10.3389/fmicb.2017.02409.

Grassi L, Maisetta G, Esin S, Batoni G. Combination strategies to enhance the efficacy of antimicrobial peptides against bacterial biofilms. Front Microbiol. 2017:8:2409.

Gries CM, Kielian T. Staphylococcal biofilms and immune polarization during prosthetic joint infection. J Am Acad Orthop Surg. 2017;25:S20.

Grivennikov SI, Greten FR, Karin M. Immunity, inflammation, and cancer. Cell. 2010;140:883-99.

Guan X, Hou Y, Sun F, Yang Z, Li C. Dysregulated chemokine signaling in cystic fibrosis lung disease: a potential therapeutic target. Curr Drug Targets. 2016:17:1535-44.

Guiducci C, Vicari AP, Sangaletti S, Trinchieri G, Colombo MP. Redirecting in vivo elicited tumor infiltrating macrophages and dendritic cells towards tumor rejection. Can Res. 2005;65:3437-46.

Guilhen C, Forestier C, Balestrino D. Biofilm dispersal: multiple elaborate strategies for dissemination of bacteria with unique properties. Mol Microbiol. 2017:105:188-210.

Guo C, et al. Immunometabolism: a new target for improving cancer immunotherapy. Adv Cancer Res. 2019;143:195-253.

Guo C, et al. (2019) Immunometabolism: a new target for improving cancer immunotherapy. In: Adv Cancer Res. Elsevier, pp. 195-253

Guss AM, et al. Phylogenetic and metabolic diversity of bacteria associated with cystic fibrosis. ISME J. 2011;5:20-9.

Guy B. The perfect mix: recent progress in adjuvant research. Nat Rev Microbiol. 2007;5:396-7.

Haabeth OAW, et al. Inflammation driven by tumour-specific Th1 cells protects against B-cell cancer. Nat Commun. 2011;2:1-12.

Hakansson A, Orihuela C, Bogaert D. Bacterial-host interactions: physiology and pathophysiology of respiratory infection. Physiol Rev. 2018;98:781-811.

Hammami I, et al. Immunosuppressive activity enhances central carbon metabolism and bioenergetics in myeloid-derived suppressor cells in vitro models. BMC Cell Biol. 2012:13:18.

Hanke ML, Kielian T. Deciphering mechanisms of staphylococcal biofilm evasion of host immunity. Front Cell Infect Microbiol. 2012;2:62.

Hanke ML, Angle A, Kielian T. MyD88-dependent signaling influences fibrosis and alternative macrophage activation during Staphylococcus aureus biofilm infection. PLOS ONE. 2012;7:e42476.

Hanke ML, Heim CE, Angle A, Sanderson SD, Kielian T. Targeting macrophage activation for the prevention and treatment of Staphylococcus aureus biofilm infections. J Immunol (baltimore, Md: 1950). 2013;190:2159-68
Hanke ML, Heim CE, Angle A, Sanderson SD, Kielian T. Targeting macrophage activation for the prevention and treatment of Staphylococcus aureus biofilm infections. J Immunol. 2013:190:2159-68.

Hänsch GM (2012) Host defence against bacterial biofilms:"Mission impossible"? ISRN Immunol. 2012

Hansen M, Andersen MH (2017) The role of dendritic cells in cancer. In: Seminars in immunopathology. Springer, pp. 307-316

Hanses F, Park S, Rich J, Lee JC. Reduced neutrophil apoptosis in diabetic mice during staphylococcal infection leads to prolonged Tnfa production and reduced neutrophil clearance. PLoS ONE. 2011;6:e23633.

Hao N-B, et al (2012) Macrophages in tumor microenvironments and the progression of tumors. Clin Dev Immunol 2012.

Haque M, Sartelli M, McKimm J, Abu BM. Health care-associated infectionsan overview. Infect Drug Resist. 2018;11:2321-33.

Harley C, Pini S, Bartlett YK, Velikova G. Defining chronic cancer: patient experiences and self-management needs. BMJ Support Palliat Care. 2012;2:248-55.

Harrison C, Bastan R, Peirce M, Munday M, Peachell P. Role of calcineurin in the regulation of human lung mast cell and basophil function by cyclosporine and FK506. Br J Pharmacol. 2007:150:509-18.

Hathroubi S, Servetas SL, Windham I, Merrell DS, Ottemann KM. Helicobacter pylori biofilm formation and its potential role in pathogenesis. Microbiol Mol Biol Rev. 2018;82:e00001-00018.

Heim CE, et al. Myeloid-derived suppressor cells contribute to Staphylococcus aureus orthopedic biofilm infection. J Immunol. 2014;192:3778-92.

Heim CE, Vidlak D, Kielian T. Interleukin-10 production by myeloid-derived suppressor cells contributes to bacterial persistence during Staphylococcus aureus orthopedic biofilm infection. J Leukoc Biol. 2015;98:1003-13.

Heim CE, et al. IL-12 promotes myeloid-derived suppressor cell recruitment and bacterial persistence during Staphylococcus aureus orthopedic implant infection. J Immunol. 2015;194:3861-72.

Heim CE, et al. Human prosthetic joint infections are associated with myeloidderived suppressor cells (MDSCs): implications for infection persistence. J Orthop Res. 2018;36:1605-13.

Heim CE, West SC, Ali H, Kielian T. Heterogeneity of Ly6G+ Ly6C+ myeloidderived suppressor cell infiltrates during Staphylococcus aureus biofilm infection. Infect Immunity. 2018. https://doi.org/10.1128/IAl.00684-18.

Heim CE, et al. Lactate production by Staphylococcus aureus biofilm inhibits HDAC11 to reprogramme the host immune response during persistent infection. Nat Microbiol. 2020:5:1271-84

Henze A-T, Mazzone M. The impact of hypoxia on tumor-associated macrophages. J Clin Investig. 2016:126:3672-9.

Herber DL, et al. Lipid accumulation and dendritic cell dysfunction in cancer. Nat Med. 2010;16:880.

Hiramatsu K, et al. N1, N12-Diacetylspermine as a sensitive and specific novel marker for early-and late-stage colorectal and breast cancers. Clin Cancer Res. 2005:11:2986-90.

Hirayama A, et al. Quantitative metabolome profiling of colon and stomach cancer microenvironment by capillary electrophoresis time-of-flight mass spectrometry. Can Res. 2009;69:4918-25.

Hirayama D, lida T, Nakase H. The phagocytic function of macrophage-enforcing innate immunity and tissue homeostasis. Int J Mol Sci. 2017;19:92.

Hossain $\mathrm{F}$, et al. Inhibition of fatty acid oxidation modulates immunosuppressive functions of myeloid-derived suppressor cells and enhances cancer therapies. Cancer Immunol Res. 2015:3:1236-47.

Housman G, et al. Drug resistance in cancer: an overview. Cancers (basel). 2014;6:1769-92.

Houston N, et al. Sputum neutrophils in cystic fibrosis patients display a reduced respiratory burst. J Cystic Fibrosis. 2013;12:352-62.

Hu W, et al. Comprehensive landscape of extracellular vesicle-derived RNAs in cancer initiation, progression, metastasis and cancer immunology. Mol Cancer. 2020:19:1-23.

Huang J, Brumell JH. Bacteria-autophagy interplay: a battle for survival. Nat Rev Microbiol. 2014:12:101.

Huang N, Perl A. Metabolism as a target for modulation in autoimmune diseases. Trends Immunol. 2018:39:562-76.

Husain Z, Huang Y, Seth P, Sukhatme VP. Tumor-derived lactate modifies antitumor immune response: effect on myeloid-derived suppressor cells and NK cells. J Immunol. 2013;191:1486-95. 
Hussan H, Clinton SK, Roberts K, Bailey MT. Fusobacterium's link to colorectal neoplasia sequenced: a systematic review and future insights. World J Gastroenterol. 2017;23:8626-50.

lannitti RG, et al. Th17/Treg imbalance in murine cystic fibrosis is linked to indoleamine 2, 3-dioxygenase deficiency but corrected by kynurenines. Am J Respir Crit Care Med. 2013;187:609-20.

Injarabian L, Devin A, Ransac S, Marteyn BS. Neutrophil metabolic shift during their lifecycle: impact on their survival and activation. Int J Mol Sci. 2019;21:287.

Ivanenko N. Biofilm and tumor: interpretation of interaction and treatment strategies. Review. Med Sci Ukraine (MSU). 2021;17:104-20.

Jablonska E, Piotrowski L, Jablonski J, Grabowska Z. VEGF in the culture of PMN and the serum in oral cavity cancer patients. Oral Oncol. 2002;38:605-9.

Jellusova J, et al. Gsk3 is a metabolic checkpoint regulator in B cells. Nat Immunol. 2017;18:303-12

Jensen $P \varnothing$, et al. Rapid necrotic killing of polymorphonuclear leukocytes is caused by quorum-sensing-controlled production of rhamnolipid by Pseudomonas aeruginosa. Microbiology. 2007;153:1329-38.

Jensen $P \varnothing$, Givskov M, Bjarnsholt T, Moser C. The immune system vs. Pseudomonas aeruginosa biofilms. FEMS Immunol Med Microbiol. 2010;59:292-305

Jesaitis AJ, et al. Compromised host defense on Pseudomonas aeruginosa biofilms: characterization of neutrophil and biofilm interactions. J Immunol. 2003;171:4329-39.

Jiang Y, Geng M, Bai L. Targeting biofilms therapy: current research strategies and development hurdles. Microorganisms. 2020;8:1222.

Jiang Z, Nero T, Mukherjee S, Olson R, Yan J. Searching for the secret of stickiness: how biofilms adhere to surfaces. Front Microbiol. 2021. https:// doi.org/10.3389/fmicb.2021.686793.

Johansson M, DeNardo DG, Coussens LM. Polarized immune responses differentially regulate cancer development. Immunol Rev. 2008;222:145-54.

Kanazawa M, et al. Effects of PSK on T and dendritic cells differentiation in gastric or colorectal cancer patients. Anticancer Res. 2005;25:443-9.

Karampoor S, et al. HIV-1 Tat protein attenuates the clinical course of experimental autoimmune encephalomyelitis (EAE). Int Immunopharmacol. 2020;78:105943.

Karampoor S, et al. Maraviroc attenuates the pathogenesis of experimental autoimmune encephalitis. Int Immunopharmacol. 2020;80:106138.

Karampoor S, et al. A possible pathogenic role of Syndecan-1 in the pathogenesis of coronavirus disease 2019 (COVID-19). Int Immunopharmacol. 2021;97:107684

Karosi T, Csomor P, Hegyi Z, Sziklai I. The presence of CD209 expressing dendritic cells correlates with biofilm positivity in chronic rhinosinusitis with nasal polyposis. Eur Arch Otorhinolaryngol. 2013;270:2455-63.

Kaufmann SHE, Kabelitz D. (2002) Introduction: the immune response to infectious agents. In: Methods in Microbiology. Academic Press, pp. 1-20

Kelly B, O'Neill LAJ. Metabolic reprogramming in macrophages and dendritic cells in innate immunity. Cell Res. 2015;25:771-84.

Khanna S, et al. Macrophage dysfunction impairs resolution of inflammation in the wounds of diabetic mice. PLOS ONE. 2010;5:e9539.

Kim JS, Okamoto K, Rubin BK. Pulmonary function is negatively correlated with sputum inflammatory markers and cough clearability in subjects with cystic fibrosis but not those with chronic bronchitis. Chest. 2006;129:1148-54

Kinder SA, Holt SC. Localization of the Fusobacterium nucleatum T18 adhesin activity mediating coaggregation with Porphyromonas gingivalis T22. J Bacteriol. 1993;175:840-50.

Kojima $\mathrm{H}$, et al. Differentiation stage-specific requirement in hypoxia-inducible factor-1a-regulated glycolytic pathway during murine B cell development in bone marrow. J Immunol. 2010;184:154-63.

Kolenbrander PE, Andersen RN, Moore LV. Coaggregation of Fusobacterium nucleatum, Selenomonas flueggei, Selenomonas infelix, Selenomonas noxia, and Selenomonas sputigena with strains from 11 genera of oral bacteria. Infect Immun. 1989:57:3194-203.

Koliarakis I, et al. Oral bacteria and intestinal dysbiosis in colorectal cancer. Int J Mol Sci. 2019;20:4146.

Kolpen M, et al. Polymorphonuclear leucocytes consume oxygen in sputum from chronic Pseudomonas aeruginosa pneumonia in cystic fibrosis. Thorax. 2010;65:57-62.

Koo H, Falsetta ML, Klein MI. The exopolysaccharide matrix: a virulence determinant of cariogenic biofilm. J Dent Res. 2013;92:1065-73.
Koo H, Allan RN, Howlin RP, Stoodley P, Hall-Stoodley L. Targeting microbial biofilms: current and prospective therapeutic strategies. Nat Rev Microbiol. 2017:15:740-55.

Kumar S, Dikshit M. Metabolic insight of neutrophils in health and disease. Front Immunol. 2019:10:2099-2099.

Kvich L, Burmølle M, Bjarnsholt T, Lichtenberg M. Do mixed-species biofilms dominate in chronic infections?-need for in situ visualization of bacterial organization. Front Cell Infect Microbiol. 2020. https://doi.org/10. 3389/fcimb.2020.00396.

Lampropoulou V, et al. Itaconate links inhibition of succinate dehydrogenase with macrophage metabolic remodeling and regulation of inflammation. Cell Metab. 2016;24:158-66.

Langowski JL, et al. IL-23 promotes tumour incidence and growth. Nature. 2006:442:461-5

Larsen T, Fiehn NE. Dental biofilm infections-an update. APMIS 2017:125:376-84

Laval J, et al. Metabolic adaptation of neutrophils in cystic fibrosis airways involves distinct shifts in nutrient transporter expression. J Immunol (baltimore, Md: 1950). 2013;190:6043-50.

Lawrence RH, Sorrelli TC. Decreased polymorphonuclear leucocyte chemotactic response to leukotriene B4 in cystic fibrosis. Clin Exp Immunol. 1992:89:321-4.

Lazar $\vee$, et al. Aspects of gut microbiota and immune system interactions in infectious diseases, immunopathology and cancer. Front Immunol. 2018:9:1830.

Le KY, Park MD, Otto M. Immune evasion mechanisms of Staphylococcus epidermidis biofilm infection. Front Microbiol. 2018:9:359-359.

Lebeaux D, Ghigo J-M, Beloin C. Biofilm-related infections: bridging the gap between clinical management and fundamental aspects of recalcitrance toward antibiotics. Microbiol Mol Biol Rev. 2014;78:510-43.

Lee NA, Gelfand EW, Lee JJ. Pulmonary T cells and eosinophils: coconspirators or independent triggers of allergic respiratory pathology? J Allergy Clin Immunol. 2001;107:945-57.

Leid JG, et al. The exopolysaccharide alginate protects Pseudomonas aeruginosa biofilm bacteria from IFN- $\gamma$-mediated macrophage killing. Immunol. 2005:175:7512-8.

Levy BD, Clish CB, Schmidt B, Gronert K, Serhan CN. Lipid mediator class switching during acute inflammation: signals in resolution. Nat Immunol. 2001;2:612-9.

Li Y-H, Tian X. Quorum sensing and bacterial social interactions in biofilms. Sensors (basel). 2012;12:2519-38.

Lighvani AA, et al. T-bet is rapidly induced by interferon- $\gamma$ in lymphoid and myeloid cells. Proc Natl Acad Sci. 2001;98:15137-42.

Lin J, Ren J, Gao DS, Dai Y, Yu L. The emerging application of itaconate: promising molecular targets and therapeutic opportunities. Front Chem. 2021. https://doi.org/10.3389/fchem.2021.669308.

Lippitz BE. Cytokine patterns in patients with cancer: a systematic review. Lancet Oncol. 2013;14:e218-28.

Liu G, et al. SIRT1 limits the function and fate of myeloid-derived suppressor cells in tumors by orchestrating HIF-1a-dependent glycolysis. Can Res. 2014;74:727-37.

Loftus RM, Finlay DK. Immunometabolism: cellular metabolism turns immune regulator. J Biol Chem. 2016;291:1-10.

Lone AG, et al. Staphylococcus aureus induces hypoxia and cellular damage in porcine dermal explants. Infect Immun. 2015;83:2531-41.

Lu J, et al. Discrete functions of M2a and M2c macrophage subsets determine their relative efficacy in treating chronic kidney disease. Kidney Int. 2013:84:745-55.

Lupp C, et al. Host-mediated inflammation disrupts the intestinal microbiota and promotes the overgrowth of Enterobacteriaceae. Cell Host Microbe. 2007;2:119-29.

Ma Q. Polarization of immune cells in the pathologic response to inhaled particulates. Front Immunol. 2020;11:1060.

Maali Y, Journo C, Mahieux R, Dutartre H. Microbial biofilms: human T-cell leukemia virus type 1 first in line for viral biofilm but far behind bacterial biofilms. Front Microbiol. 2020. https://doi.org/10.3389/fmicb.2020. 02041.

Maali Y, Journo C, Mahieux R, Dutartre H. Microbial biofilms: human T-cell leukemia virus type 1 first in line for viral biofilm but far behind bacterial biofilms. Front Microbiol. 2020;11:2041-2041. 
Macintyre AN, et al. The glucose transporter Glut1 is selectively essential for CD4 T cell activation and effector function. Cell Metab. 2014;20:61-72.

Maclver NJ, Michalek RD, Rathmell JC. Metabolic regulation of T lymphocytes. Annu Rev Immunol. 2013;31:259-83.

Madsen JS, Burmølle M, Hansen LH, Sørensen SJ. The interconnection between biofilm formation and horizontal gene transfer. FEMS Immunol Med Microbiol. 2012;65:183-95.

Mahdiun F, Mansouri S, Khazaeli P, Mirzaei R. The effect of tobramycin incorporated with bismuth-ethanedithiol loaded on niosomes on the quorum sensing and biofilm formation of Pseudomonas aeruginosa. Microb Pathog. 2017;107:129-35.

Mahmoud S, et al. (2011) Tumor-infiltrating CD8. J Clin Oncol 29

Makam M, et al. Activation of critical, host-induced, metabolic and stress pathways marks neutrophil entry into cystic fibrosis lungs. Proc Natl Acad Sci USA. 2009;106:5779-83.

Mandell GL. Bactericidal activity of aerobic and anaerobic polymorphonuclear neutrophils. Infect Immun. 1974;9:337-41.

Mantovani A, Biswas SK, Galdiero MR, Sica A, Locati M. Macrophage plasticity and polarization in tissue repair and remodelling. J Pathol. 2013:229:176-85.

Markman M. Commentary: implications of cancer managed as a "chronic illness." Curr Oncol Rep. 2011;13:90-1.

Martinez FO, Gordon S. The M1 and M2 paradigm of macrophage activation: time for reassessment. F1000 Prime Rep. 2014;6:13.

Martínez-Reyes I, Chandel NS. Mitochondrial TCA cycle metabolites control physiology and disease. Nat Commun. 2020;11:102.

Martins M, et al. The Pil3 pilus of Streptococcus gallolyticus binds to intestinal mucins and to fibrinogen. Gut Microbes. 2016;7:526-32.

Mashimo C, et al. Identification of the genes involved in the biofilm-like structures on actinomyces oris $\mathrm{K20}$, a clinical isolate from an apical lesion. J Endodontics. 2013;39:44-8.

Masucci MT, Minopoli M, Carriero MV. Tumor associated neutrophils. their role in tumorigenesis, metastasis, prognosis and therapy. Front Oncol. 2019;9:1146-1146.

Maurice NM, Bedi B, Sadikot RT. Pseudomonas aeruginosa biofilms: host response and clinical implications in lung infections. Am J Respir Cell Mol Biol. 2018:58:428-39.

Mazumdar C, Driggers EM, Turka LA. The untapped opportunity and challenge of immunometabolism: a new paradigm for drug discovery. Cell Metab. 2020:31:26-34.

McColl KE. Clinical practice. Helicobacter pylori infection. N Engl J Med. 2010;362:1597-604

Menter T, Tzankov A. Mechanisms of immune evasion and immune modulation by lymphoma cells. Front Oncol. 2018. https://doi.org/10.3389/ fonc.2018.00054.

Mentis A-FA, Boziki M, Grigoriadis N, Papavassiliou AG. Helicobacter pylori infection and gastric cancer biology: tempering a double-edged sword. Cell Mol Life Sci. 2019;76:2477-86.

Michalek RD, et al. Cutting edge: distinct glycolytic and lipid oxidative metabolic programs are essential for effector and regulatory CD4+ T cell subsets. J Immunol. 2011;186:3299-303.

Mirzaei R, Sadeghi J, Talebi M, Irajian G. Prevalence of atIE, ica, mecA, and mupA genes in Staphylococcus epidermidis isolates. Infect Dis Clin Pract. 2017;25:37-40.

Mirzaei $\mathrm{R}$, et al. The importance of intracellular bacterial biofilm in infectious diseases. Microbial Pathog. 2020a. https://doi.org/10.1016/j.micpath. 2020.104393.

Mirzaei R, Abdi M, Gholami H. The host metabolism following bacterial biofilm: what is the mechanism of action? Rev Med Microbiol. 2020b;31:175-82.

Mirzaei R, et al. The biofilm-associated bacterial infections unrelated to indwelling devices. IUBMB Life. 2020c;72:1271-85.

Mirzaei R, et al. Overview of the current promising approaches for the development of an effective severe acute respiratory syndrome coronavirus 2 (SARS-CoV-2) vaccine. Int Immunopharmacol. 2020d;106928:106928.

Mirzaei $\mathrm{R}$, et al. Bacterial biofilm in colorectal cancer: what is the real mechanism of action? Microbial Pathog. 2020e;142:104052.

Mirzaei R, et al. Role of microRNAs in Staphylococcus aureus infection: potential biomarkers and mechanism. IUBMB Life. 2020f;72:1856-69.
Mirzaei R, et al. Role of microbiota-derived short-chain fatty acids in cancer development and prevention. Biomed Pharmacotherapy. 2021a;139:111619.

Mirzaei R, et al. Role of microbiota-derived short-chain fatty acids in nervous system disorders. Biomed Pharmacotherapy. 2021b;139:111661.

Mirzaei R, et al. The emerging role of exosomal miRNAs as a diagnostic and therapeutic biomarker in Mycobacterium tuberculosis infection. Mol Med. 2021c;27:1-31.

Mirzaei $R$, et al. The pathogenic, therapeutic and diagnostic role of exosomal microrna in the autoimmune diseases. J Neuroimmunol. 2021d;358:577640.

Mirzaei R, et al. Dual role of microbiota-derived short-chain fatty acids on host and pathogen. Biomed Pharmacotherapy. 2021e;145:112352.

Mirzaei R, et al. Immunometabolism in human brucellosis: an emerging field of investigation. Microbial Pathog. 2021f;158:105115.

Mirzaei R, et al. (2021g) The emerging role of probiotics as a mitigation strategy against coronavirus disease 2019 (COVID-19). Arch Virol. 1-22

Mirzaei R, Ranjbar R. Hijacking host components for bacterial biofilm formation: an advanced mechanism. Int Immunopharmacol. 2022;103:108471.

Mithieux G. Gut microbiota and host metabolism: what relationship. Neuroendocrinology. 2018;106:352-6.

Mockler MB, Conroy MJ, Lysaght J. Targeting T cell immunometabolism for cancer immunotherapy; understanding the impact of the tumor microenvironment. Front Oncol. 2014;4:107.

Molinero N, Ruiz L, Sánchez B, Margolles A, Delgado S. Intestinal bacteria interplay with bile and cholesterol metabolism: implications on host physiology. Front Physiol. 2019. https://doi.org/10.3389/fphys.2019. 00185.

Molinero N, Ruiz L, Sánchez B, Margolles A, Delgado S. Intestinal Bacteria interplay with bile and cholesterol metabolism: implications on host physiology. Front Physiol. 2019;10:185.

Muenst S, et al. The immune system and cancer evasion strategies: therapeutic concepts. J Intern Med. 2016;279:541-62.

Murray PJ. The primary mechanism of the IL-10-regulated antiinflammatory response is to selectively inhibit transcription. Proc Natl Acad Sci. 2005;102:8686-91.

Murray PJ, et al. Macrophage activation and polarization: nomenclature and experimental guidelines. Immunity. 2014;41:14-20.

Nagaraj S, Gabrilovich DI. Tumor escape mechanism governed by myeloidderived suppressor cells. Can Res. 2008;68:2561-3.

Narsale A, Moya R, Davies JD. Human CD4+ CD25+ CD127hi cells and the Th1/Th2 phenotype. Clin Immunol. 2018;188:103-12.

$\mathrm{Ng}$ CT, Snell LM, Brooks DG, Oldstone MBA. Networking at the level of host immunity: immune cell interactions during persistent viral infections. Cell Host Microbe. 2013;13:652-64.

Nguyen KT, et al. Deficient cytokine expression and neutrophil oxidative burst contribute to impaired cutaneous wound healing in diabetic, biofilmcontaining chronic wounds. Wound Repair Regen. 2013;21:833-41.

Nicklin P, et al. Bidirectional transport of amino acids regulates mTOR and autophagy. Cell. 2009;136:521-34.

Nielsen SR, Schmid MC (2017) Macrophages as key drivers of cancer progression and metastasis. Mediators Inflamm 2017.

Nilsson C, et al. Correlation between cag pathogenicity island composition and Helicobacter pylori-associated gastroduodenal disease. Infect Immun. 2003;71:6573-81.

Noman MZ, et al. PD-L1 is a novel direct target of HIF-1a, and its blockade under hypoxia enhanced MDSC-mediated T cell activation. J Exp Med. 2014:211:781-90.

Noy R, Pollard JW. Tumor-associated macrophages: from mechanisms to therapy. Immunity. 2014;41:49-61.

O'Neill LA, Pearce EJ. Immunometabolism governs dendritic cell and macrophage function. J Exp Med. 2016;213:15-23.

Onizuka S, et al. Tumor rejection by in vivo administration of anti-CD25 (interleukin-2 receptor alpha) monoclonal antibody. Cancer Res. 1999:59:3128-33.

Ost $\mathrm{M}$, et al. Myeloid-derived suppressor cells in bacterial infections. Front Cell Infect Microbiol. 2016;6:37-37. 
Ostrand-Rosenberg S, Bronte V. Coordinated regulation of myeloid cells by tumours. Nat Rev Immunol. 2012;12:253-68.

Ostrand-Rosenberg S, Sinha P. Myeloid-derived suppressor cells: linking inflammation and cancer. J Immunol. 2009;182:4499-506.

Ouyang W, et al. Stat6-independent GATA-3 autoactivation directs IL4-independent Th2 development and commitment. Immunity. 2000;12:27-37.

Özen S, Berk Ö, Şimşek DG, Darcan S. Clinical course of Hashimoto's thyroiditis and effects of levothyroxine therapy on the clinical course of the disease in children and adolescents. J Clin Res Pediatr Endocrinol. 2011;3:192-7.

Palmieri EM, et al. Acetylation of human mitochondrial citrate carrier modulates mitochondrial citrate/malate exchange activity to sustain NADPH production during macrophage activation. Biochimica Et Biophysica Acta (BBA) Bioenergetics. 2015;1847:729-38.

Pan J, et al. Immune responsive gene 1, a novel oncogene, increases the growth and tumorigenicity of glioma. Oncol Rep. 2014;32:1957-66.

Paraschos S, et al. In vitro and in vivo activities of Chios mastic gum extracts and constituents against Helicobacter pylori. Antimicrob Agents Chemother. 2007:51:551-9.

Parkin DM. The global health burden of infection-associated cancers in the year 2002. Int J Cancer. 2006;118:3030-44.

Parks QM, et al. Neutrophil enhancement of Pseudomonas aeruginosa biofilm development: human F-actin and DNA as targets for therapy. J Med Microbiol. 2009:58:492-502.

Pavlova NN, Thompson CB. The emerging hallmarks of cancer metabolism. Cell Metab. 2016:23:27-47.

Pearce EL, Pearce EJ. Metabolic pathways in immune cell activation and quiescence. Immunity. 2013;38:633-43.

Percival SL, Malic S, Cruz H, Williams DW (2011) Introduction to biofilms. In: Biofilms and veterinary medicine. Springer. pp. 41-68

Percival SL, Suleman L. Biofilms and Helicobacter pylori: dissemination and persistence within the environment and host. World J Gastrointestinal Pathophysiol. 2014;5:122-32.

Petrova V, Annicchiarico-Petruzzelli M, Melino G, Amelio I. The hypoxic tumour microenvironment. Oncogenesis. 2018;7:10-10.

Pizzoli SFM, Renzi C, Arnaboldi P, Russell-Edu W, Pravettoni G. From lifethreatening to chronic disease: Is this the case of cancers? A systematic review. Cogent Psychology. 2019;6:1577593.

Polat IH. (2016) Functional role of pentose phosphate pathway and glutamine in cancer cell metabolism

Porta C, et al. Tolerance and M2 (alternative) macrophage polarization are related processes orchestrated by p50 nuclear factor KB. Proc Natl Acad Sci. 2009;106:14978-83.

Prabhakara R, et al. Suppression of the inflammatory immune response prevents the development of chronic biofilm infection due to methicillinresistant Staphylococcus aureus. Infect Immun. 2011;79:5010-8.

Prendergast GC, Malachowski WP, DuHadaway JB, Muller AJ. Discovery of IDO1 inhibitors: from bench to bedside. Can Res. 2017:77:6795-811.

Proctor RA, et al. Small colony variants: a pathogenic form of bacteria that facilitates persistent and recurrent infections. Nat Rev Microbiol. 2006:4:295.

Proietti E, et al. Type I IFN as a natural adjuvant for a protective immune response: lessons from the influenza vaccine model. J Immunol. 2002;169:375-83.

Protti MP, De Monte L. Thymic stromal lymphopoietin and cancer: Th2Dependent and-independent mechanisms. Front Immunol. 2020. https://doi.org/10.3389/fimmu.2020.02088.

Rada B. Interactions between neutrophils and Pseudomonas aeruginosa in cystic fibrosis. Pathogens (Basel, Switzerland). 2017;6:10

Rada B. Interactions between neutrophils and Pseudomonas aeruginosa in cystic fibrosis. Pathogens. 2017;6:10.

Ranjbar R, Karampoor S, Jalilian FA. The protective effect of Helicobacter Pylori infection on the susceptibility of multiple sclerosis. J Neuroimmunol. 2019:337:577069.

Rasamiravaka T, Labtani Q, Duez P, El Jaziri M. The formation of biofilms by Pseudomonas aeruginosa: a review of the natural and synthetic compounds interfering with control mechanisms. BioMed Res Int. 2015;2015:759348.

Rasamiravaka T, Labtani Q, Duez P, El Jaziri M (2015) The formation of biofilms by Pseudomonas aeruginosa: a review of the natural and synthetic compounds interfering with control mechanisms. BioMed Res Int 2015.
Rasoul M, Rokhsareh M, Mohammad SM, Sajad K, Ahmadreza M. The human immune system against Staphylococcus epidermidis. Crit Rev Immunol. 2019:39:151.

Reiner SL. Epigenetic control in the immune response. Hum Mol Genet. 2005;14:R41-6.

Renner K, et al. Metabolic plasticity of human T cells: preserved cytokine production under glucose deprivation or mitochondrial restriction, but 2-deoxy-glucose affects effector functions. Eur J Immunol. 2015:45:2504-16.

Riera-Domingo C, et al. Immunity, hypoxia, and metabolism-the Ménage à Trois of cancer: implications for immunotherapy. Physiol Rev. 2020;100:1-102.

Ripoli M, et al. Hepatitis C virus-linked mitochondrial dysfunction promotes hypoxia-inducible factor 1a-mediated glycolytic adaptation. J Virol. 2010;84:647-60.

Riquelme SA, et al. Pseudomonas aeruginosa utilizes host-derived itaconate to redirect its metabolism to promote biofilm formation. Cell Metab. 2020:31:1091-1106.e1096

Rizzato C, et al. Potential role of biofilm formation in the development of digestive tract cancer with special reference to Helicobacter pylori infection. Front Microbiol. 2019;10:846.

Roilides E, Simitsopoulou M, Katragkou A, Walsh TJ (2015) How biofilms evade host defenses. Microbial Biofilms 287-300

Ruangsetakit C, Chinsakchai K, Mahawongkajit P, Wongwanit C, Mutirangura P. Transcutaneous oxygen tension: a useful predictor of ulcer healing in critical limb ischaemia. J Wound Care. 2010;19:202-6.

Saab M-B, et al (2010) Morphological differences between normal and cancerous mammalian cells via multitechnique microscopic studies. In: Biophotonics: Photonic Solutions for Better Health Care II. International Society for Optics and Photonics, p. 771518

Sadowska B, Więckowska-Szakiel M, Paszkiewicz M, Różalska B. The immunomodulatory activity of Staphylococcus aureus products derived from biofilm and planktonic cultures. Arch Immunol Ther Exp. 2013;61:413-20.

Sasikaran J, Ziemski M, Zadora PK, Fleig A, Berg IA. Bacterial itaconate degradation promotes pathogenicity. Nat Chem Biol. 2014;10:371-7.

Savijoki K, et al. Growth mode and physiological state of cells prior to biofilm formation affect immune evasion and persistence of Staphylococcus aureus. Microorganisms. 2020;8:106.

Scarlett UK, et al. Ovarian cancer progression is controlled by phenotypic changes in dendritic cells. J Exp Med. 2012;209:495-506.

Scherr TD, et al. Global transcriptome analysis of Staphylococcus aureus biofilms in response to innate immune cells. Infect Immun. 2013;81:4363-76.

Scherr TD, Heim CE, Morrison JM, Kielian T. Hiding in plain sight: interplay between Staphylococcal biofilms and host immunity. Front Immunol. 2014:5:37.

Scherr TD, et al. Staphylococcus aureus biofilms induce macrophage dysfunction through leukocidin $A B$ and alpha-toxin. Mbio. 2015;6:e01021-e1015.

Scherr TD, et al. (2015) Staphylococcus aureus biofilms induce macrophage dysfunction through leukocidin $A B$ and alpha-toxin. MBio 6

Schmielau J, Finn OJ. Activated granulocytes and granulocyte-derived hydrogen peroxide are the underlying mechanism of suppression of t-cell function in advanced cancer patients. Can Res. 2001:61:4756-60.

Schönfeld P, Wojtczak L. Short- and medium-chain fatty acids in energy metabolism: the cellular perspective. J Lipid Res. 2016;57:943-54.

Selvan R, S, P Dowling J, K Kelly W, Lin J. Indoleamine 2, 3-dioxygenase (IDO): biology and target in cancer immunotherapies. Curr Cancer Drug Targets. 2016;16:755-64.

Servetas SL, et al. Characterization of key Helicobacter pylori regulators identifies a role for ArsRS in biofilm formation. J Bacteriol. 2016:198:2536-48.

Shao C, et al. Changes of proteome components of Helicobacter pylori biofilms induced by serum starvation. Mol Med Rep. 2013;8:1761-6.

Shen X, et al. Calcineurin inhibitors cyclosporin A and tacrolimus protect against podocyte injury induced by puromycin aminonucleoside in rodent models. Sci Rep. 2016;6:1-16. 
Sherene G-T, Loi SG, Equeter C, Libin M, de Wind A, Ravoet M, et al. CD4+ follicular helper T cell infiltration predicts breast cancer survival. J Clin Investig. 2013;123:2873-92.

Sheu B-C, et al. Predominant Th2/Tc2 polarity of tumor-infiltrating lymphocytes in human cervical cancer. J Immunol. 2001;167:2972-8.

Shimizu J, Yamazaki S, Sakaguchi S. Induction of tumor immunity by removing CD25+CD4+ T cells: a common basis between tumor immunity and autoimmunity. J Immunol (baltimore, Md: 1950). 1999;163:5211-8.

Shimizu J, Yamazaki S, Sakaguchi S. Induction of tumor immunity by removing CD25+ CD4+ T cells: a common basis between tumor immunity and autoimmunity. J Immunol. 1999;163:5211-8.

Shirtliff ME, Mader JT, Camper AK. Molecular interactions in biofilms. Chem Biol. 2002;9:859-71.

Shkreta L, Talbot BG, Diarra MS, Lacasse P. Immune responses to a DNA/protein vaccination strategy against Staphylococcus aureus induced mastitis in dairy cows. Vaccine. 2004;23:114-26.

Sibley $C D$, et al. Culture enriched molecular profiling of the cystic fibrosis airway microbiome. PLOS ONE. 2011;6:e22702.

Sica A, Mantovani A. Macrophage plasticity and polarization: in vivo veritas. J Clin Investig. 2012;122:787-95.

Sica A, Mantovani A. Macrophage plasticity and polarization: in vivo veritas. J Clin Invest. 2012;122:787-95.

Singer K, Cheng WC, Kreutz M, Ho PC, Siska PJ. Immunometabolism in cancer at a glance. Dis Models Mech. 2018. https://doi.org/10.1242/dmm. 034272 .

Singer K, Cheng W-C, Kreutz M, Ho P-C, Siska PJ. Immunometabolism in cancer at a glance. Dis Models Mech. 2018:11:34272.

Sinha P, Clements VK, Ostrand-Rosenberg S. Reduction of myeloid-derived suppressor cells and induction of M1 macrophages facilitate the rejection of established metastatic disease. J Immunol. 2005;174:636-45.

Sinha P. Clements VK, Bunt SK, Albelda SM, Ostrand-Rosenberg S. Crosstalk between myeloid-derived suppressor cells and macrophages subverts tumor immunity toward a type 2 response. J Immunol. 2007;179:977-83.

Siska PJ, Rathmell JC. T cell metabolic fitness in antitumor immunity. Trends Immunol. 2015;36:257-64.

Slack M, Wang T, Wang R. T cell metabolic reprogramming and plasticity. Mol Immunol. 2015:68:507-12.

Slavik JM, Hutchcroft JE, Bierer BE. CD80 and CD86 are not equivalent in their ability to induce the tyrosine phosphorylation of CD28. J Biol Chem. 1999;274:3116-24.

Som P, et al. A fluorinated glucose analog, 2-fluoro-2-deoxy-D-glucose (F-18): nontoxic tracer for rapid tumor detection. J Nucl Med. 1980;21:670-5.

Souto JC, Vila L, Bru A. Polymorphonuclear neutrophils and cancer: intense and sustained neutrophilia as a treatment against solid tumors. Med Res Rev. 2011;31:311-63.

Stewart PS. Diffusion in biofilms. J Bacteriol. 2003;185:1485-91.

Stewart PS. Antimicrobial tolerance in biofilms. Microbiol Spectrum. 2015. https://doi.org/10.1128/microbiolspec.MB-0010-2014.

Stoodley P, Sauer K, Davies DG, Costerton JW. Biofilms as complex differentiated communities. Ann Rev Microbiol. 2002:56:187-209.

Sun J, Kato I. Gut microbiota, inflammation and colorectal cancer. Genes Dis. 2016:3:130-43.

Sutmuller RP, et al. Synergism of cytotoxic T lymphocyte-associated antigen 4 blockade and depletion of CD25+ regulatory T cells in antitumor therapy reveals alternative pathways for suppression of autoreactive cytotoxic T lymphocyte responses. J Exp Med. 2001;194:823-32.

Swidsinski A, Weber J, Loening-Baucke V, Hale LP, Lochs H. Spatial organization and composition of the mucosal flora in patients with inflammatory bowel disease. J Clin Microbiol. 2005:43:3380-9.

Szabo SJ, Jacobson NG, Dighe AS, Gubler U, Murphy KM. Developmental commitment to the Th2 lineage by extinction of IL-12 signaling. Immunity. 1995;2:665-75.

Tada K, et al. Pretreatment immune status correlates with progressionfree survival in chemotherapy-treated metastatic colorectal cancer patients. Cancer Immunol Res. 2016:4:592-9.

Taga K, Mostowski H, Tosato G. Human interleukin-10 can directly inhibit T-cell growth. Blood. 1993;81:2964.

Tan T-T, Coussens LM. Humoral immunity, inflammation and cancer. Curr Opin Immunol. 2007;19:209-16.
Tang A, et al. Inflammasome-mediated IL-1 $\beta$ production in humans with cystic fibrosis. PLoS ONE. 2012;7:e37689.

Tannahill $\mathrm{G}$, et al. Succinate is an inflammatory signal that induces IL-1 $\beta$ through HIF-1 a. Nature. 2013;496:238-42.

Tateda K, et al. The Pseudomonas aeruginosa autoinducer N-3-oxododecanoyl homoserine lactone accelerates apoptosis in macrophages and neutrophils. Infect Immun. 2003:71:5785-93.

Tcyganov E, Mastio J, Chen E, Gabrilovich DI. Plasticity of myeloid-derived suppressor cells in cancer. Curr Opin Immunol. 2018;51:76-82.

Thai M, et al. Adenovirus E4ORF1-induced MYC activation promotes host cell anabolic glucose metabolism and virus replication. Cell Metab. 2014;19:694-701.

Thurlow LR, et al. Staphylococcus aureus biofilms prevent macrophage phagocytosis and attenuate inflammation in vivo. J Immunol. 2011;186:6585-96.

Tomkovich S, Jobin C. Microbial networking in cancer: when two toxins collide. Berlin: Nature Publishing Group; 2018.

Tomkovich S, et al. Human colon mucosal biofilms from healthy or colon cancer hosts are carcinogenic. J Clin Invest. 2019;129:1699-712.

Tomlinson KL, Riquelme SA. Host-bacteria metabolic crosstalk drives S. aureus biofilm. Microb Cell. 2021;8:106-7.

Tomlinson KL, et al. Staphylococcus aureus induces an itaconate-dominated immunometabolic response that drives biofilm formation. Nat Commun. 2021;12:1399.

Torres A, Makowski L, Wellen KE. Immunometabolism: metabolism finetunes macrophage activation. Elife. 2016;5:e14354.

Tucci M, et al. Immune system evasion as hallmark of melanoma progression: the role of dendritic cells. Front Oncol. 2019;9:1148.

Tunney MM, et al. Detection of anaerobic bacteria in high numbers in sputum from patients with cystic fibrosis. Am J Respir Crit Care Med. 2008:177:995-1001.

van Gennip M, et al. Interactions between polymorphonuclear leukocytes and Pseudomonas aeruginosa biofilms on silicone implants in vivo. Infect Immun. 2012;80:2601-7.

Van Overmeire E, et al. M-CSF and GM-CSF receptor signaling differentially regulate monocyte maturation and macrophage polarization in the tumor microenvironment. Can Res. 2016;76:35-42.

Vander Heiden MG, DeBerardinis RJ. Understanding the intersections between metabolism and cancer biology. Cell. 2017;168:657-69.

Vander Heiden MG, Cantley LC, Thompson CB. Understanding the Warburg effect: the metabolic requirements of cell proliferation. Science (new York, n.y). 2009:324:1029-33.

Vikström E, Magnusson K-E, Pivoriūnas A. The Pseudomonas aeruginosa quorum-sensing molecule $\mathrm{N}$-(3-oxododecanoyl)-L-homoserine lactone stimulates phagocytic activity in human macrophages through the p38 MAPK pathway. Microbes Infect. 2005;7:1512-8.

Vitale I, Manic G, Coussens LM, Kroemer G, Galluzzi L. Macrophages and metabolism in the tumor microenvironment. Cell Metab. 2019:30:36-50

Vitko NP, Spahich NA, Richardson AR. Glycolytic dependency of high-level nitric oxide resistance and virulence in Staphylococcus aureus. Mbio. 2015;6:e00045-e115.

Voelxen NF, et al. Comparative metabolic analysis in head and neck cancer and the normal gingiva. Clin Oral Invest. 2018;22:1033-43.

Volfson D, Cookson S, Hasty J, Tsimring LS. Biomechanical ordering of dense cell populations. Proc Natl Acad Sci. 2008;105:15346-51.

Walker TS, et al. Enhanced Pseudomonas aeruginosa biofilm development mediated by human neutrophils. Infect Immun. 2005;73:3693-701.

Wang R, Green DR. Metabolic reprogramming and metabolic dependency in T cells. Immunol Rev. 2012;249:14-26.

Wang $\mathrm{YC}$, et al. Notch signaling determines the M1 versus M2 polarization of macrophages in antitumor immune responses. Cancer Res. 2010;70:4840-9.

Wang $\mathrm{Q}$ et al. The anti-inflammatory drug dimethyl itaconate protects against colitis-associated colorectal cancer. J Mol Med. 2020;98:1457-66.

Warburg O. Uber den stoffwechsel der karzinomezellen. Biochem Z. 1924;152:309-44.

Warburg O, Wind F, Negelein E. The metabolism of tumors in the body. J Gen Physiol. 1927;8:519-30. 
Ward-Hartstonge K, Kemp R. Regulatory T-cell heterogeneity and the cancer immune response. Clin Transl Immunol. 2017;6:e154.

Watters C, Everett JA, Haley C, Clinton A, Rumbaugh KP. Insulin treatment modulates the host immune system to enhance Pseudomonas aeruginosa wound biofilms. Infect Immun. 2014;82:92-100.

Watters C, Yuan TT, Rumbaugh KP (2015) Beneficial and deleterious bacterialhost interactions in chronic wound pathophysiology. University of Texas at Austin Austin United States

Wculek SK, et al. Dendritic cells in cancer immunology and immunotherapy. Nat Rev Immunol. 2020;20:7-24.

Weagel E, Smith C, Liu P, Robison R, O'Neill K. Macrophage polarization and its role in cancer. J Clin Cell Immunol. 2015:6:338.

Weaver CT, Harrington LE, Mangan PR, Gavrieli M, Murphy KM. Th17: an effector CD4 T cell lineage with regulatory T cell ties. Immunity. 2006;24:677-88

Weiss JM, et al. Itaconic acid mediates crosstalk between macrophage metabolism and peritoneal tumors. J Clin Investig. 2018;128:3794-805.

Williams NC, O'Neill LA. A role for the krebs cycle intermediate citrate in metabolic reprogramming in innate immunity and inflammation. Front Immunol. 2018:9:141.

Wittekind C, Neid M. Cancer invasion and metastasis. Oncology. 2005;69:14-6.

Wong EH, et al. Comparative genomics revealed multiple Helicobacter pylori genes associated with biofilm formation in vitro. PLOS ONE. 2016;11:e0166835.

Worlitzsch D, et al. Effects of reduced mucus oxygen concentration in airway Pseudomonas infections of cystic fibrosis patients. J Clin Investig. 2002;109:317-25.

Wu J, Horuzsko A. Expression and function of immunoglobulin-like transcripts on tolerogenic dendritic cells. Hum Immunol. 2009;70:353-6.

Wu Y, Klapper I, Stewart PS. Hypoxia arising from concerted oxygen consumption by neutrophils and microorganisms in biofilms. Pathogens Dis. 2018;76:fty043.

Wyckoff J, et al. A paracrine loop between tumor cells and macrophages is required for tumor cell migration in mammary tumors. Can Res. 2004;64:7022-9.

Wyckoff JB, et al. Direct visualization of macrophage-assisted tumor cell intravasation in mammary tumors. Can Res. 2007;67:2649-56.

Xu H-M. Th1 cytokine-based immunotherapy for cancer. Hepatobiliary Pancreat Dis Int. 2014;13:482-94.

Yamada KJ, et al. (2018) Arginase-1 expression in myeloid cells regulates Staphylococcus aureus planktonic but not biofilm infection. Infect Immunity 86

Yamada KJ, Kielian T. Biofilm-leukocyte cross-talk: impact on immune polarization and immunometabolism. J Innate Immun. 2019;11:280-8.

Yamada KJ, et al. Monocyte metabolic reprogramming promotes pro-inflammatory activity and Staphylococcus aureus biofilm clearance. PLoS Pathog. 2020;16:e1008354-e1008354.

Yan J, Bassler BL. Surviving as a community: antibiotic tolerance and persistence in bacterial biofilms. Cell Host Microbe. 2019;26:15-21.

Yanagida O, et al. Human L-type amino acid transporter 1 (LAT1): characterization of function and expression in tumor cell lines. Biochem Biophys Acta. 2001;1514:291-302.

Yang J, Murphy TL, Ouyang W, Murphy KM. Induction of interferon- $\gamma$ production in Th1 CD4+ T cells: evidence for two distinct pathways for promoter activation. Eur J Immunol. 1999;29:548-55.

Yang $W$, et al. Potentiating the antitumour response of CD8+ T cells by modulating cholesterol metabolism. Nature. 2016;531:651-5.

Yordanov D, Boyanova L. Influence of dietary factors on Helicobacter Pylori and CagA seroprevalence in Bulgaria. Gastroenterol Res Pract. 2017;2017:9212143.

Youn JI, Collazo M, Shalova IN, Biswas SK, Gabrilovich DI. Characterization of the nature of granulocytic myeloid-derived suppressor cells in tumorbearing mice. J Leukoc Biol. 2012;91:167-81.

Yu H, et al. Type III secretion protein, PcrV, impairs Pseudomonas aeruginosa biofilm formation by increasing M1 macrophage-mediated anti-bacterial activities. Front Microbiol. 2020;11:1971.

Yuan Y, Jiang Y-C, Sun C-K, Chen Q-M. Role of the tumor microenvironment in tumor progression and the clinical applications. Oncol Rep. 2016;35:2499-515.

Zhang $\mathrm{M}$, et al. Interactions between intestinal microbiota and host immune response in inflammatory bowel disease. Front Immunol. 2017;8:942.
Zhao X, et al. Saikosaponin A inhibits breast cancer by regulating Th1/Th2 balance. Front Pharmacol. 2019;10:624.

Zhou J, Nefedova Y, Lei A, Gabrilovich D (2018) Neutrophils and PMN-MDSC: their biological role and interaction with stromal cells. In: Seminars in immunology. Elsevier, pp. 19-28

Zhu L, Zhao Q, Yang T, Ding W, Zhao Y. Cellular metabolism and macrophage functional polarization. Int Rev Immunol. 2015;34:82-100.

Zou W. Immunosuppressive networks in the tumour environment and their therapeutic relevance. Nat Rev Cancer. 2005:5:263-74.

Zoumot Z, Wilson R. Respiratory infection in noncystic fibrosis bronchiectasis. Curr Opin Infect Dis. 2010;23:165-70.

\section{Publisher's Note}

Springer Nature remains neutral with regard to jurisdictional claims in published maps and institutional affiliations.

Ready to submit your research? Choose BMC and benefit from:

- fast, convenient online submission

- thorough peer review by experienced researchers in your field

- rapid publication on acceptance

- support for research data, including large and complex data types

- gold Open Access which fosters wider collaboration and increased citations

- maximum visibility for your research: over $100 \mathrm{M}$ website views per year

At BMC, research is always in progress.

Learn more biomedcentral.com/submissions 